\title{
Nonlocal Numerical Simulation of Low Reynolds Number Laminar Fluid Motion by Using Peridynamic Differential Operator
}

\author{
Yan Gao and Selda Oterkus* \\ Department of Naval Architecture, Ocean and Marine Engineering \\ University of Strathclyde, 100 Montrose Street, Glasgow, G4 OLZ, United Kingdom
}

\begin{abstract}
A considerable fluid load can cause local damages on the offshore structures, which may be a risk in the field of ocean engineering. Therefore, an accurate fluid motion prediction is a crucial issue in predicting the offshore structure motion. In this study, a non-local Lagrangian model is developed for Newtonian fluid low Reynold's number laminar flow. Based on the peridynamic theory, a peridynamic differential operator is recently proposed for directly converting the partial differential into its integral form. Therefore, the peridynamic differential operator is applied to convert the classical Navier-Stokes equations into their integral forms. The numerical algorithms are developed both in total and updated Lagrangian description. Finally, several benchmark fluid flow problems such as Couette flow, Poiseuille flow, Taylor Green vortex, shear-driven cavity problem and dam collapse problems are numerically solved. The simulation results are compared with the ones available in the published literature. The good agreements validate of the capability of the proposed non-local model for Newtonian fluid low Reynold's number laminar flow simulation.
\end{abstract}

Keywords: peridynamics differential operator, non-local, fluid flow, Lagrangian description, dam break

\section{Introduction}

The fluid and structure interaction are often encountered in ocean engineering, e.g. floating structures and water waves interactions (Faltinsen, 1993), sloshing in a water tank (Cao et al., 2014), green water impact (Gómez-Gesteira et al., 2005) etc. The fluid motion is a crucial issue because of the fluid induced movements such as heaving and rolling of the offshore structures. Sometimes the fluid may even also cause considerable pressure loadings on the offshore structure and make local damages of these structures (Faltinsen, 1993). Therefore, an

*Corresponding Author: Selda Oterkus, Department of Naval Architecture, Ocean and Marine Engineering, University of Strathclyde. Email: selda.oterkus@ strath.ac.uk. 
accurate fluid motion prediction is necessary for a fluid-structure interaction simulation in the field of ocean engineering. Peridynamics (Silling, 2000) has been applied on the numerical simulations of offshore structure damage in the ocean engineering field, e.g. the ship-ice interaction (Liu et al., 2018; Wang et al., 2018; Ye et al., 2017). It will be beneficial to simulate both the structure and fluid with the same methodology, e.g. peridynamics. Therefore, a peridynamic fluid model is provided in this paper which can be further incorporated into the peridynamic solid model to simulate the fluid and structure interactions.

The Eulerian approach is adopted in most computational fluid dynamics (CFD) methodologies. Since CFD can solve fluid flow problems with any boundary and initial conditions, it has been widely used in academic research (Anderson and Wendt, 1995). There are two types of computational Lagrangian approaches: total Lagrangian approach and updated Lagrangian approach. The total Lagrangian approach uses the initial configuration as the reference configuration, while the updated Lagrangian approach adopts the current configuration as the reference configuration. Smoothed Particle Hydrodynamics (SPH), which is an updated Lagrangian approach, is another widely used method for fluid flow simulations (Gingold and Monaghan, 1977; Lucy, 1977). SPH is a mesh-free particle method, and it does not need a grid to calculate spatial derivatives (Monaghan, 1992). In addition, it is easy to work with and can give reasonable accuracy. Therefore, it draws a lot of attention in recent years.

Peridynamics (PD) is another mesh-free particle method, which is initially formulated as a total Lagrangian description. It is introduced (Silling, 2000) for solid mechanics. The PD replaces the partial differential equations of the classical continuum mechanics by integral equations (Silling and Askari, 2005). Since integral equations remain valid even at discontinuities, singular stress generated by the classical continuum mechanics is avoided. Therefore, PD is well suited for solving the problems with discontinuities, e.g. crack propagation simulations. Since the PD is originally proposed for structural mechanical problems, it is generally applied to predict fracture in solids (Madenci and Oterkus, 2014; Oterkus and Madenci, 2012a; Oterkus and Madenci, 2012b). Hence the application of PD in fluid mechanics is still in its infant stage. Only a few PD fluid models are available in the published literature. A state-based peridynamic formulation is presented by Katiyar et al. (2014) to simulate the fluid flow in porous media. Later on, the model is applied to simulate the growth of fluid driven cracks in porous and fracture media (Ouchi et al., 2015). A fully coupled poroelastic peridynamic formulation is introduced by Oterkus et al. (2017) to simulate fluidfilled fractures. In their model, the coupling effect of porous fluid flow and deformation of porous media is considered to predict the behaviour of fluid-filled fractures. However, in these 
PD models the fluid flow was limited to porous flow. Therefore, the developed PD fluid models cannot be utilized for general fluid flow simulations. An Eulerian form of peridynamic model is presented to by Silling et al. (2017) to simulate the shockwaves. In their model, the peridynamic forces are defined in the deformed configuration to simulate strong shock waves and fluid response for very large deformations. An updated lagrangian PD model based on the state-based peridynamics concept is applied as updated Lagrangian particle hydrodynamics (ULPH) to simulate Newtonian fluid flow (Tu and Li, 2017).

The bond-based peridynamics (Silling, 2000), referred as an original PD theory, assumes the pairwise PD force densities being equal in magnitude and parallel in direction. The interaction of each PD bond is independent of the other PD bonds within the integration domain. Consequently, there is a limitation on the material properties for bond-based PD theory. Later on, the state-based PD theory is introduced which removes the aforementioned limitation of bond-based PD theory (Silling et al., 2007). If the pairwise PD force density vectors are not equal in magnitude but still parallel in direction, the theory is classified as ordinary state-based PD theory. However, the PD form of the material parameters is required for PD material modelling, which is calculated by comparing the strain energy density obtained from classical continuum mechanics and PD expressions. If there is no constraint on the pairwise PD force density vectors, it is classified as non-ordinary state-based PD theory. The non-ordinary peridynamics can be directly related to the classical continuum model in terms of deformation gradient tensor and stress tensor (Silling et al., 2007). The presence of the zero-energy mode is a challenging issue in the non-ordinary peridynamic material model (Luo and Sundararaghavan, 2018; Silling, 2017). The displacement oscillations exist in the PD solutions especially for steep deformation gradients (Gu et al., 2018). One possible cause of the oscillations may arise from applying first order derivative approximation twice for approximating the second order derivatives (Gu et al., 2018).

Based on the PD concept, a peridynamic differential operator is recently proposed by Madenci et al. (Madenci et al., 2016). It is derived based on Taylor series expansion and orthogonal function properties. Being different from the bond-based PD theory, the peridynamic differential operator does not have any constraint on the material properties. Furthermore, it can be directly applied to reformulate the partial differential equations to their integral forms. The classical physical parameters can be directly used without converting into their PD expressions, which avoids the derivation process required by the bond based and ordinary state-based peridynamic theory. In addition, it does not have any limitation on the order of the partial derivatives both for time and space. For example, the second-order 
derivatives can be directly approximated by one integration by using the PD differential operator which corresponds to the second order derivative. As a result, the error of the PD result is reduced by using less integrations, compared to non-ordinary state based PD. Furthermore, the PD differential operator functions are also forced to be orthogonal to each term in the Taylor series expansion (Madenci et al., 2016). Therefore, when determining the expressions of the PD differential operators, both lower and higher order terms are considered. In this study, peridynamic differential operator is used to simulate low Reynolds number flow problems.

This paper is organized as follows. Section 2 describes the peridynamic differential operator including its properties. Section 3 describes the developed non-local velocity operator by using the peridynamic differential operator. Section 4 describes the non-local form of Navier-Stokes equations that are derived based on their local forms by using non-local velocity operator. Section 5 presents the numerical algorithms both in total and updated Lagrangian descriptions. In addition, the treatments regarding the numerical simulation are explained. Section 6 presents a set of classical fluid flow problems, i.e. Couette flow, Poiseuille flow, Taylor Green vortex, shear driven cavity, and dam collapse problems are numerically simulated with the developed model. Finally, the conclusions are given in Section 7.

\section{Peridynamic Differential Operator}

Peridynamics is a non-local theory which provides an alternative formulation for the continuum mechanics. PD is based on integral-differential equations as opposed to the partial differential equations of classical continuum mechanics (Silling, 2000). Here, the basic concepts of the PD theory are reviewed briefly (Madenci and Oterkus, 2014). As shown in Fig. 1 , the whole body, $R$, is discretized into a finite set of sub-regions for computer simulations. The sub-regions are represented by the PD nodes identified by their initial locations, e.g. $\mathbf{x}\left(x_{1}, x_{2}, x_{3}\right)$. Each PD node, $\mathbf{x}$, interacts with all other PD nodes, $\mathbf{x}^{\prime}\left(x_{1}^{\prime}, x_{2}^{\prime}, x_{3}^{\prime}\right)$, within a distance $\delta$. Here, $\mathbf{x}$ is the point of interest and $\mathbf{x}^{\prime}$ is called a family member of $\mathbf{x}$. The interaction domain, $H_{\mathbf{x}}$, is the neighbourhood of $\mathbf{x}$ and its radius $\delta$ is named horizon. The length of the horizon is the measure of nonlocal behaviour. The initial relative position between $\mathbf{x}$ and $\mathbf{x}^{\prime}$ is denoted by $\xi$, i.e. $\mathbf{x}^{\prime}=\mathbf{x}+\xi$. The acceleration of the central point is calculated by the force exerted from its family members, indicating a non-local behaviour. 


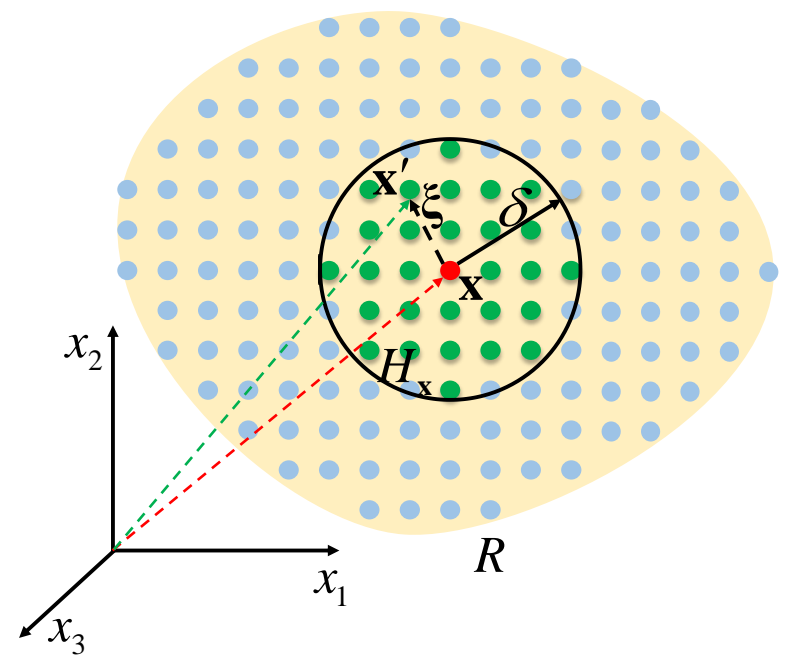

Fig. 1 Interaction of the point of interest $\mathbf{x}$ with its family member $\mathbf{x}^{\prime}$

In light of the PD concepts, a PD differential operator is proposed by Madenci et al. (Madenci et al., 2016) and is fully explained in this section. One advantage of the PD differential operator is that the classical material parameters can be directly applied in the nonlocal integral equations (Madenci et al., 2016). Many physical fields, including the fluid flow field, involve up to second order partial derivatives with respect to spatial variables. Therefore, the PD differential operator corresponding to up to second order derivatives in a threedimensional space is adopted in this study. The PD differential operator property is constructed based on the Taylor series expansion and the orthogonal function properties, which will be explained in Section 2.1. The construction of the PD differential operator is presented in Section 2.2.

\subsection{PD differential operator}

Taylor series expansion in a three-dimensional space up to second order derivatives can be introduced for a multi-variable scalar field, as

$$
f(\mathbf{x})=f\left(x_{1}, x_{2}, x_{3}\right)
$$

then the difference value between $f(\mathbf{x}+\xi)$ and $f(\mathbf{x})$ can be approximated by using Taylor series expansion with ignoring the higher order terms as

$$
\begin{aligned}
f(\mathbf{x}+\xi)-f(\mathbf{x}) & =\xi_{1} \frac{\partial f(\mathbf{x})}{\partial x_{1}}+\xi_{2} \frac{\partial f(\mathbf{x})}{\partial x_{2}}+\xi_{3} \frac{\partial f(\mathbf{x})}{\partial x_{3}}+\xi_{1} \xi_{2} \frac{\partial^{2} f(\mathbf{x})}{\partial x_{1} \partial x_{2}}+\xi_{1} \xi_{3} \frac{\partial^{2} f(\mathbf{x})}{\partial x_{1} \partial x_{3}} \\
& +\xi_{2} \xi_{3} \frac{\partial^{2} f(\mathbf{x})}{\partial x_{2} \partial x_{3}}+\frac{1}{2} \xi_{1}^{2} \frac{\partial^{2} f(\mathbf{x})}{\partial x_{1}^{2}}+\frac{1}{2} \xi_{2}^{2} \frac{\partial^{2} f(\mathbf{x})}{\partial x_{2}^{2}}+\frac{1}{2} \xi_{3}^{2} \frac{\partial^{2} f(\mathbf{x})}{\partial x_{3}^{2}}+R_{\mathbf{x}, 2}(\xi)
\end{aligned}
$$

or expressed in a compact form as 


$$
f(\mathbf{x}+\xi)-f(\mathbf{x})=\sum_{n_{1}=0}^{2} \sum_{n_{2}=0}^{2-n_{1}} \sum_{n_{3}=0}^{2-n_{1}-n_{2}} \frac{1}{n_{1} ! n_{2} ! n_{3} !} \xi_{1}^{n_{1}} \xi_{2}^{n_{2}} \xi_{3}^{n_{3}} \frac{\partial^{n_{1}+n_{2}+n_{3}} f(\mathbf{x})}{\partial x_{1}^{n_{1}} \partial x_{2}^{n_{2}} \partial x_{3}^{n_{3}}}+R_{\mathbf{x}, 2}(\xi)
$$

where $0<n_{1}+n_{2}+n_{3} \leq 2$. The integer terms $n_{1}, n_{2}$, and $n_{3}$ represent the differential order of $f(\mathbf{x})$ with respect to $x_{1}, x_{2}$, and $x_{3}$, respectively. The term $R_{\mathbf{x}, 2}(\xi)$ represents the remainder and it is assumed to be negligibly small. Therefore, the term $f(\mathbf{x}+\xi)-f(\mathbf{x})$ is an approximation whose error is proportional to $|\xi|^{3}$. The term $\xi$ represents the relative position vector between two material points as

$$
\xi=\xi_{1} \mathbf{e}_{1}+\xi_{2} \mathbf{e}_{2}+\xi_{3} \mathbf{e}_{3}
$$

with

$$
\begin{aligned}
& \xi_{1}=\left(x_{1}^{\prime}-x_{1}\right) \\
& \xi_{2}=\left(x_{2}^{\prime}-x_{3}\right) \\
& \xi_{3}=\left(x_{3}^{\prime}-x_{3}\right)
\end{aligned}
$$

The terms $\mathbf{e}_{1}, \mathbf{e}_{2}$, and $\mathbf{e}_{3}$ represent the unit vectors in $x_{1}, x_{2}$, and $x_{3}$ directions. The scalar values $\xi_{1}, \xi_{2}$, and $\xi_{3}$ represent the projections of the relative position vector on the corresponding unit vectors.

As with a basis of vectors in a finite-dimensional space, orthogonal functions can form an infinite basis for a function space. Orthogonal functions can be treated in analogy with the approach of expressing a location of a node with a vector set $\left(\mathbf{e}_{1}, \mathbf{e}_{2}, \mathbf{e}_{3}\right)$ for a threedimensional space.

The PD differential operators (Madenci et al., 2016) are used to represent the PD nonlocal expressions for the partial derivatives. The derivative up to second derivative can be denoted as

$$
\frac{\partial^{p_{1}+p_{2}+p_{3}} f(\mathbf{x})}{\partial x_{1}^{p_{1}} \partial x_{2}^{p_{2}} \partial x_{3}^{p_{3}}}
$$

where $0<p_{1}+p_{2}+p_{3} \leq 2$. The term $p_{i}$ represents the order of differentiation with respect to $x_{i}$, with $i=1,2,3$. Therefore, function $g^{p_{1} p_{2} p_{3}}(\xi)$ is used to represent the PD nonlocal expressions up to the second derivative. For example, $g^{101}(\xi)$ represents the PD operator for derivative $\partial^{1+0+1} f(\mathbf{x}) / \partial x_{1}^{1} \partial x_{2}^{0} \partial x_{3}^{1}$. Furthermore, the set of the PD differential operator is considered to be a set of functions that are orthogonal to the exponent of relative positions, 
$\xi_{1}^{n_{1}} \xi_{2}^{n_{2}} \xi_{3}^{n_{3}}$. Therefore, the PD differential operator $g^{p_{1} p_{2} p_{3}}(\xi)$ possess the orthogonality property as (Madenci et al., 2016)

$$
\frac{1}{n_{1} ! n_{2} ! n_{3} !} \int_{H_{\mathrm{x}}} \xi_{1}^{n_{1}} \xi_{2}^{n_{2}} \xi_{3}^{n_{3}} g^{p_{1} p_{2} p_{3}}(\xi) \mathrm{d} V^{\prime}=\delta_{n_{1} p_{1}} \delta_{n_{2} p_{2}} \delta_{n_{3} p_{3}}
$$

where $\delta_{n_{i} p_{i}}$ is the Kronecker delta with $i=1,2,3$.

Based on Taylor series expansion and orthogonal function properties, the property of the PD differential operator can be derived. Multiplying the PD differential operator $g^{p_{1} p_{2} p_{3}}(\xi)$ on both sides of Eq. (2b), and performing an integration over the horizon domain of central point $\mathbf{x}$ results in (Madenci et al., 2016)

$$
\begin{aligned}
& \int_{H_{\mathbf{x}}}(f(\mathbf{x}+\xi)-f(\mathbf{x})) g^{p_{1} p_{2} p_{3}}(\xi) \mathrm{d} V^{\prime} \\
& =\int_{H_{\mathbf{x}}}\left(g^{p_{1} p_{2} p_{3}}(\xi) \sum_{n_{1}=0}^{2} \sum_{n_{2}=0}^{2-n_{1}} \sum_{n_{3}=0}^{2-n_{1}-n_{2}} \frac{1}{n_{1} ! n_{2} ! n_{3} !} \xi_{1}^{n_{1}} \xi_{2}^{n_{2}} \xi_{3}^{n_{3}} \frac{\partial^{n_{1}+n_{2}+n_{3}} f(\mathbf{x})}{\partial x_{1}^{n_{1}} \partial x_{2}^{n_{2}} \partial x_{3}^{n_{3}}}\right) \mathrm{d} V^{\prime}
\end{aligned}
$$

After applying the linear property of integration on the right-hand side of Eq. (7), it results in

$$
\begin{aligned}
& \int_{H_{\mathbf{x}}}(f(\mathbf{x}+\xi)-f(\mathbf{x})) g^{p_{1} p_{2} p_{3}}(\xi) \mathrm{d} V^{\prime} \\
& =\sum_{n_{1}=0}^{2} \sum_{n_{2}=0}^{2-n_{1}} \sum_{n_{3}=0}^{2-n_{1}-n_{2}} \frac{1}{n_{1} ! n_{2} ! n_{3} !} \int_{H_{\mathbf{x}}} \frac{\partial^{n_{1}+n_{2}+n_{3}} f(\mathbf{x})}{\partial x_{1}^{n_{1}} \partial x_{2}^{n_{2}} \partial x_{3}^{n_{3}}} \xi_{1}^{n_{1}} \xi_{2}^{n_{2}} \xi_{3}^{n_{3}} g^{p_{1} p_{2} p_{3}}(\xi) \mathrm{d} V^{\prime}
\end{aligned}
$$

By using the orthogonal property described in Eq. (6) , Eq. (8) becomes (Madenci et al., 2016)

$$
\begin{aligned}
& \int_{H_{\mathbf{x}}}(f(\mathbf{x}+\boldsymbol{\xi})-f(\mathbf{x})) g^{p_{1} p_{2} p_{3}}(\boldsymbol{\xi}) \mathrm{d} V^{\prime} \\
& =\frac{1}{p_{1} ! p_{2} ! p_{3} !} \int_{H_{\mathbf{x}}}\left(\frac{\partial^{p_{1}+p_{2}+p_{3}} f(\mathbf{x})}{\partial x_{1}^{p_{1}} \partial x_{2}^{p_{2}} \partial x_{3}^{p_{3}}} \xi_{1}^{p_{1}} \xi_{2}^{p_{2}} \xi_{3}^{p_{3}} g^{p_{1} p_{2} p_{3}}(\xi)\right) \mathrm{d} V^{\prime} \\
& =\frac{\partial^{p_{1}+p_{2}+p_{3}} f(\mathbf{x})}{\partial x_{1}^{p_{1}} \partial x_{2}^{p_{2}} \partial x_{3}^{p_{3}}}
\end{aligned}
$$

or in an explicit form (Madenci et al., 2016) 


$$
\left\{\begin{array}{l}
\frac{\partial f(\mathbf{x})}{\partial x_{1}} \\
\frac{\partial f(\mathbf{x})}{\partial x_{2}} \\
\frac{\partial f(\mathbf{x})}{\partial x_{3}} \\
\frac{\partial^{2} f(\mathbf{x})}{\partial x_{1}^{2}} \\
\frac{\partial^{2} f(\mathbf{x})}{\partial x_{2}^{2}} \\
\frac{\partial^{2} f(\mathbf{x})}{\partial x_{3}^{2}} \\
\frac{\partial^{2} f(\mathbf{x})}{\partial x_{1} \partial x_{2}} \\
\frac{\partial^{2} f(\mathbf{x})}{\partial x_{1} \partial x_{3}} \\
\frac{\partial^{2} f(\mathbf{x})}{\partial x_{2} \partial x_{3}}
\end{array}\right\}=\int_{H_{\mathbf{x}}}(f(\mathbf{x}+\xi)-f(\mathbf{x}))\left\{\begin{array}{l}
g^{100}(\xi) \\
g^{010}(\xi) \\
g^{001}(\xi) \\
g^{200}(\xi) \\
g^{020}(\xi) \\
g^{002}(\xi) \\
g^{110}(\xi) \\
g^{101}(\xi) \\
g^{011}(\xi)
\end{array}\right\} \mathrm{d} V^{\prime}
$$

In this way, the partial derivatives of the central point can be represented by an integration of function and the corresponding PD differential operator over the horizon as shown in Eq. (10). In other words, with the help of PD differential operator, partial derivatives can be converted to their integral forms (Madenci et al., 2016).

\subsection{Construction of PD differential operator}

The PD differential operator functions, $g^{p_{1} p_{2} p_{3}}(\xi)$, can be constructed as (Madenci et al., 2016)

$$
g^{p_{1} p_{2} p_{3}}(\xi)=\sum_{q_{1}=0}^{2} \sum_{q_{2}=0}^{2-q_{1}} \sum_{q_{3}=0}^{2-q_{1}-q_{2}} a_{q_{1} q_{2} q_{3}}^{p_{1} p_{2} p_{3}} w(|\xi|) \xi_{1}^{q_{1}} \xi_{2}^{q_{2}} \xi_{3}^{q_{3}}
$$

where $w(|\xi|)$ represents the weight function and $a_{q_{1} q_{2} q_{3}}^{p_{1} p_{2} p_{3}}$ represents the unknown coefficient matrix with $0<q_{1}+q_{2}+q_{3} \leq 2$. In this study, a weighted function is defined as a Gaussian distribution which depends on the relative position and the horizon as (Madenci et al., 2016)

$$
w(|\xi|)=e^{-(2|\xi| \delta)^{2}}
$$


The derivation of the unknown coefficient $a_{q_{1} q_{2} q_{3}}^{p_{1} p_{2} p_{3}}$ in Eq. (11) can be derived by using orthogonality property provided in Eq. (6) (Madenci et al., 2016).

By substituting Eq. (11) into Eq. (6), the following equation can be obtained as

$$
\frac{1}{n_{1} ! n_{2} ! n_{3} !} \int_{H_{x}} \xi_{1}^{n_{1}} \xi_{2}^{n_{2}} \xi_{3}^{n_{3}} \sum_{q_{1}=0}^{2} \sum_{q_{2}=0}^{2-q_{1}} \sum_{q_{3}=0}^{2-q_{1}-q_{2}} a_{q_{1} q_{2} q_{3}}^{p_{1} p_{2} p_{3}} w(|\xi|) \xi_{1}^{q_{1}} \xi_{2}^{q_{2}} \xi_{3}^{q_{3}} \mathrm{~d} V^{\prime}=\delta_{n_{1} p_{1}} \delta_{n_{2} p_{2}} \delta_{n_{3} p_{3}}
$$

After applying the linear property of integration, Eq. (13) results in

$$
\sum_{q_{1}=0}^{2} \sum_{q_{2}=0}^{2-q_{1}} \sum_{q_{3}=0}^{2-q_{1}-q_{2}}\left(\left(\int_{H_{\mathbf{x}}} \xi_{1}^{n_{1}+q_{1}} \xi_{2}^{n_{2}+q_{2}} \xi_{3}^{n_{3}+q_{3}} w(|\xi|) \mathrm{d} V^{\prime}\right) a_{q_{1} q_{2} q_{3}}^{p_{1} p_{2} p_{3}}\right)=n_{1} ! n_{2} ! n_{3} ! \delta_{n_{1} p_{1}} \delta_{n_{2} p_{2}} \delta_{n_{3} p_{3}}
$$

Therefore, the unknown term, $a_{q_{1} q_{2} q_{3}}^{p_{1} p_{2} p_{3}}$, can be obtained by the following equation

$$
\sum_{q_{1}=0}^{2} \sum_{q_{2}=0}^{2-q_{1}} \sum_{q_{3}=0}^{2-q_{1}-q_{2}} A_{\left(n_{1} n_{2} n_{3}\right)\left(q_{1} q_{2} q_{3}\right)} a_{q_{1} q_{2} q_{3}}^{p_{1} p_{2} p_{3}}=b_{n_{1} n_{2} n_{3}}^{p_{1} p_{3} p_{3}}
$$

with

$$
A_{\left(n_{1} n_{2} n_{3}\right)\left(q_{1} q_{2} q_{3}\right)}=\int_{H_{\mathbf{x}}} \xi_{1}^{n_{1}+q_{1}} \xi_{2}^{n_{2}+q_{2}} \xi_{3}^{n_{3}+q_{3}} w(|\xi|) \mathrm{d} V^{\prime}
$$

and

$$
b_{n_{1} n_{2} n_{3}}^{p_{1} p_{2} p_{3}}=n_{1} ! n_{2} ! n_{3} ! \delta_{n_{1} p_{1}} \delta_{n_{2} p_{2}} \delta_{n_{3} p_{3}}
$$

The peridynamic differential operator for two-dimensional space up to second order derivative is provided in an explicit form in Appendix A.

\section{Non-local Velocity Operator by Using PD Differential Operator}

In this section, the non-local form of velocity divergence, gradient, and second derivatives are developed to approximate their corresponding local operators. The non-local derivatives will be utilized in the derivation of the non-local form of Navier-Stokes equations in Section 4. PD differential operator tensors which are expressed in the matrix form are introduced as

$$
\mathbf{g}_{1}(\xi)=\left[g^{100}(\xi) g^{010}(\xi) g^{001}(\xi)\right]
$$

and

$$
\mathbf{g}_{2}(\xi)=\left[\begin{array}{lll}
g^{200}(\xi) & g^{110}(\xi) & g^{101}(\xi) \\
g^{110}(\xi) & g^{020}(\xi) & g^{011}(\xi) \\
g^{101}(\xi) & g^{011}(\xi) & g^{002}(\xi)
\end{array}\right]
$$

where $\mathbf{g}_{1}(\xi)$ and $\mathbf{g}_{2}(\xi)$ represent the first order and second order PD differential operators up to second order derivatives, respectively. They can also be expressed as 


$$
\mathbf{g}_{1}(\xi)=\left[\begin{array}{lll}
g_{1}^{(1)}(\xi) & g_{1}^{(2)}(\xi) & g_{1}^{(3)}(\xi)
\end{array}\right]
$$

and

$$
\mathbf{g}_{2}(\xi)=\left[\begin{array}{lll}
g_{2}^{(1,1)}(\xi) & g_{2}^{(1,2)}(\xi) & g_{2}^{(1,3)}(\xi) \\
g_{2}^{(2,1)}(\xi) & g_{2}^{(2,2)}(\xi) & g_{2}^{(2,3)}(\xi) \\
g_{2}^{(3,1)}(\xi) & g_{2}^{(3,2)}(\xi) & g_{2}^{(3,3)}(\xi)
\end{array}\right]
$$

where the term $g_{1}^{(i)}(\xi)$ represents the elements in $\mathbf{g}_{1}(\xi)$ vector, $g_{2}^{(i, j)}(\xi)$ represents the elements in $\mathbf{g}_{2}(\xi)$ matrix with $i, j=1,2,3$. Therefore, the elements in Eq. (17b) represents the differential operators for second derivatives provided in Eq. (10).

Three-dimensional velocity vector for point $\mathbf{x}$ can be denoted as

$$
\mathbf{v}(\mathbf{x})=v_{1}(\mathbf{x}) \mathbf{e}_{1}+v_{2}(\mathbf{x}) \mathbf{e}_{2}+v_{3}(\mathbf{x}) \mathbf{e}_{3}
$$

where the scalar values $v_{1}(\mathbf{x}), v_{2}(\mathbf{x})$ and $v_{3}(\mathbf{x})$ represent the projections of the velocity vector on the corresponding unit vectors.

\subsection{Non-local form of velocity divergence}

The local form of velocity divergence is defined as

$$
\nabla \cdot \mathbf{v}(\mathbf{x})=\frac{\partial v_{1}(\mathbf{x})}{\partial x_{1}}+\frac{\partial v_{2}(\mathbf{x})}{\partial x_{2}}+\frac{\partial v_{3}(\mathbf{x})}{\partial x_{3}}
$$

As described by Eq. (10), by applying the first order PD differential operator, $\mathbf{g}_{1}(\xi)$, the nonlocal form of the first order derivatives can be evaluated as

$$
\begin{aligned}
& \frac{\partial v_{1}(\mathbf{x})}{\partial x_{1}}=\int_{H_{\mathbf{x}}}\left(v_{1}\left(\mathbf{x}^{\prime}\right)-v_{1}(\mathbf{x})\right) g_{1}^{(1)}(\boldsymbol{\xi}) \mathrm{d} V^{\prime} \\
& \frac{\partial v_{2}(\mathbf{x})}{\partial x_{2}}=\int_{H_{\mathbf{x}}}\left(v_{2}\left(\mathbf{x}^{\prime}\right)-v_{2}(\mathbf{x})\right) g_{1}^{(2)}(\xi) \mathrm{d} V^{\prime} \\
& \frac{\partial v_{3}(\mathbf{x})}{\partial x_{3}}=\int_{H_{\mathbf{x}}}\left(v_{3}\left(\mathbf{x}^{\prime}\right)-v_{3}(\mathbf{x})\right) g_{1}^{(3)}(\xi) \mathrm{d} V^{\prime}
\end{aligned}
$$

Therefore, the non-local form of velocity divergence can be expressed by using PD differential operator as

$$
\begin{aligned}
\nabla \cdot \mathbf{v}(\mathbf{x}) & =\int_{H_{\mathbf{x}}}\left(v_{1}\left(\mathbf{x}^{\prime}\right)-v_{1}(\mathbf{x})\right) g_{1}^{(1)}(\xi) \mathrm{d} V^{\prime}+\int_{H_{\mathbf{x}}}\left(v_{2}\left(\mathbf{x}^{\prime}\right)-v_{2}(\mathbf{x})\right) g_{1}^{(2)}(\xi) \mathrm{d} V^{\prime} \\
& +\int_{H_{\mathbf{x}}}\left(v_{3}\left(\mathbf{x}^{\prime}\right)-v_{3}(\mathbf{x})\right) g_{1}^{(3)}(\xi) \mathrm{d} V^{\prime}
\end{aligned}
$$


Eq. (21) can also be written as

$$
\nabla \cdot \mathbf{v}(\mathbf{x})=\int_{H_{\mathbf{x}}}\left[\begin{array}{l}
\left(v_{1}\left(\mathbf{x}^{\prime}\right)-v_{1}(\mathbf{x})\right) g_{1}^{(1)}(\xi) \\
+\left(v_{2}\left(\mathbf{x}^{\prime}\right)-v_{2}(\mathbf{x})\right) g_{1}^{(2)}(\xi) \\
+\left(v_{3}\left(\mathbf{x}^{\prime}\right)-v_{3}(\mathbf{x})\right) g_{1}^{(3)}(\xi)
\end{array}\right] \mathrm{d} V^{\prime}
$$

Subsequently, by considering the vector form of PD differential operator in Eq. (17a), the nonlocal form of velocity divergence can be expressed in compact form as

$$
\frac{\partial v_{i}(\mathbf{x})}{\partial x_{i}}=\int_{H_{\mathbf{x}}} g_{1}^{(i)}(\xi)\left(v_{i}\left(\mathbf{x}^{\prime}\right)-v_{i}(\mathbf{x})\right) \mathrm{d} V^{\prime}
$$

or

$$
\nabla \cdot \mathbf{v}(\mathbf{x})=\int_{H_{\mathbf{x}}} \mathbf{g}_{1}(\xi) \cdot\left(\mathbf{v}\left(\mathbf{x}^{\prime}\right)-\mathbf{v}(\mathbf{x})\right) \mathrm{d} V^{\prime}
$$

Fig. 2 presents the relative velocity vector, $\left(\mathbf{v}\left(\mathbf{x}^{\prime}\right)-\mathbf{v}(\mathbf{x})\right)$, first order PD differential operator vector, $\mathbf{g}_{1}$, and their dot products.

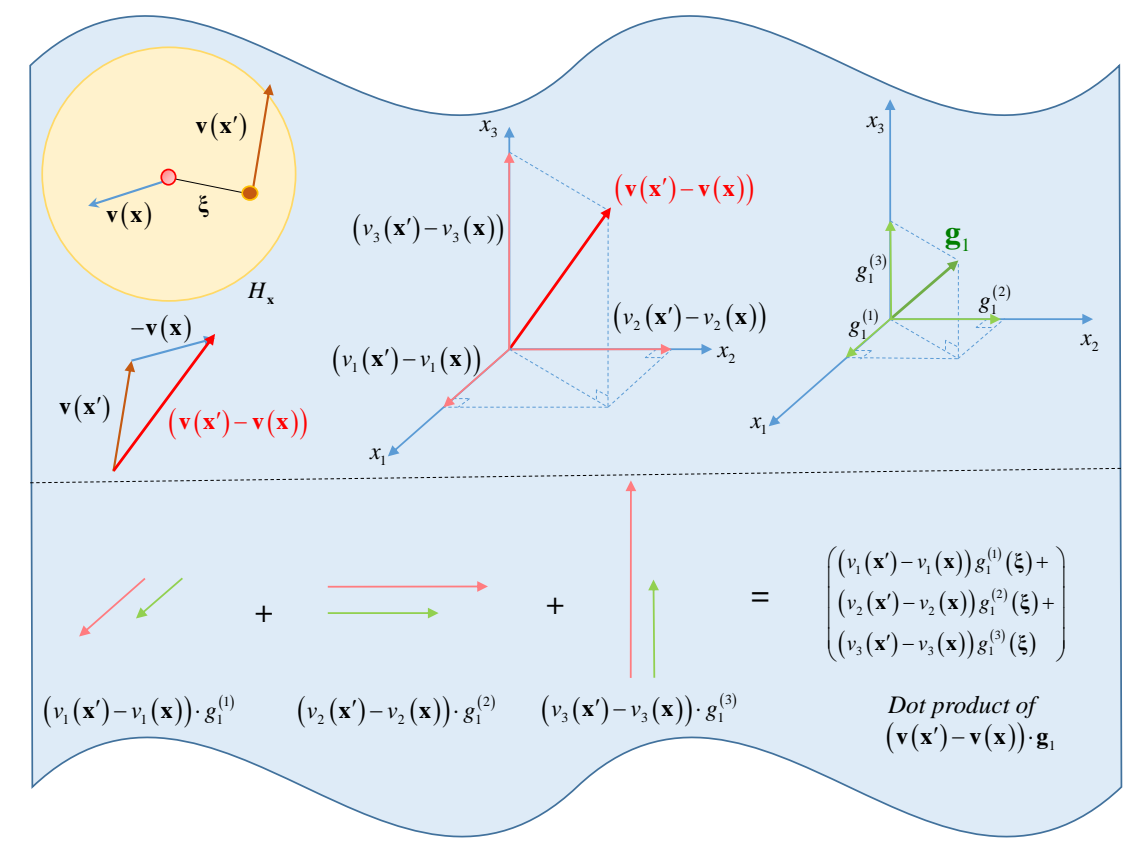

Fig. 2 Illustration of relative velocity vector $\left(\mathbf{v}\left(\mathbf{x}^{\prime}\right)-v(\mathbf{x})\right)$, first order PD differential operator vector, $\mathbf{g}_{1}$ and their dot products

3.2 Non-local form of velocity gradient

The local form of the velocity gradient is defined as 


$$
\nabla \otimes \mathbf{v}(\mathbf{x})=\frac{\partial v_{i}(\mathbf{x})}{\partial x_{j}} \mathbf{e}_{i} \otimes \mathbf{e}_{j}=\left[\begin{array}{ccc}
\frac{\partial v_{1}(\mathbf{x})}{\partial x_{1}} & \frac{\partial v_{1}(\mathbf{x})}{\partial x_{2}} & \frac{\partial v_{1}(\mathbf{x})}{\partial x_{3}} \\
\frac{\partial v_{2}(\mathbf{x})}{\partial x_{1}} & \frac{\partial v_{2}(\mathbf{x})}{\partial x_{2}} & \frac{\partial v_{2}(\mathbf{x})}{\partial x_{3}} \\
\frac{\partial v_{3}(\mathbf{x})}{\partial x_{1}} & \frac{\partial v_{3}(\mathbf{x})}{\partial x_{2}} & \frac{\partial v_{3}(\mathbf{x})}{\partial x_{3}}
\end{array}\right]
$$

where $i, j=1,2,3$. The non-local expressions of the diagonal elements in the gradient matrix are already provided in Eq. (21). Similarly, the non-local form derivatives in Eq. (24) can be expressed as

$$
\frac{\partial v_{i}(\mathbf{x})}{\partial x_{j}}=\int_{H_{\mathbf{x}}}\left(v_{i}\left(\mathbf{x}^{\prime}\right)-v_{i}(\mathbf{x})\right) g_{1}^{(j)}(\xi) \mathrm{d} V^{\prime}
$$

As a result, the non-local form for the velocity gradient can be expressed by using PD differential operator as

$$
\begin{aligned}
& \nabla \otimes \mathbf{v}(\mathbf{x}) \\
& =\int_{H_{\mathbf{x}}}\left[\begin{array}{lll}
\left(v_{1}\left(\mathbf{x}^{\prime}\right)-v_{1}(\mathbf{x})\right) g_{1}^{(1)}(\xi) & \left(v_{1}\left(\mathbf{x}^{\prime}\right)-v_{1}(\mathbf{x})\right) g_{1}^{(2)}(\xi) & \left(v_{1}\left(\mathbf{x}^{\prime}\right)-v_{1}(\mathbf{x})\right) g_{1}^{(3)}(\xi) \\
\left(v_{2}\left(\mathbf{x}^{\prime}\right)-v_{2}(\mathbf{x})\right) g_{1}^{(1)}(\xi) & \left(v_{2}\left(\mathbf{x}^{\prime}\right)-v_{2}(\mathbf{x})\right) g_{1}^{(2)}(\xi) & \left(v_{2}\left(\mathbf{x}^{\prime}\right)-v_{2}(\mathbf{x})\right) g_{1}^{(3)}(\xi) \\
\left(v_{3}\left(\mathbf{x}^{\prime}\right)-v_{3}(\mathbf{x})\right) g_{1}^{(1)}(\xi) & \left(v_{3}\left(\mathbf{x}^{\prime}\right)-v_{3}(\mathbf{x})\right) g_{1}^{(2)}(\xi) & \left(v_{3}\left(\mathbf{x}^{\prime}\right)-v_{3}(\mathbf{x})\right) g_{1}^{(3)}(\xi)
\end{array}\right] \mathrm{d} V^{\prime}
\end{aligned}
$$

Subsequently, the matrix form in Eq. (26) can be converted to a compact form as

$$
\nabla \otimes \mathbf{v}(\mathbf{x})=\int_{H_{\mathbf{x}}}\left(\mathbf{v}\left(\mathbf{x}^{\prime}\right)-\mathbf{v}(\mathbf{x})\right) \otimes \mathbf{g}_{1}(\xi) \mathrm{d} V^{\prime}
$$

\subsection{Non-local form for second derivatives of velocity}

Based on the non-local form of the first order derivative provided in Section 3.1 and 3.2, the non-local form of the second derivatives is provided in this section.

The local form of Laplacian operator is defined as

$$
\Delta \mathbf{v}=\nabla \cdot(\nabla \otimes \mathbf{v})=\left[\begin{array}{l}
\frac{\partial^{2} v_{1}}{\partial x_{1}^{2}}+\frac{\partial^{2} v_{1}}{\partial x_{2}^{2}}+\frac{\partial^{2} v_{1}}{\partial x_{3}^{2}} \\
\frac{\partial^{2} v_{2}}{\partial x_{1}^{2}}+\frac{\partial^{2} v_{2}}{\partial x_{2}^{2}}+\frac{\partial^{2} v_{2}}{\partial x_{3}^{2}} \\
\frac{\partial^{2} v_{3}}{\partial x_{1}^{2}}+\frac{\partial^{2} v_{3}}{\partial x_{2}^{2}}+\frac{\partial^{2} v_{3}}{\partial x_{3}^{2}}
\end{array}\right]
$$

As described Eq. (10), by applying the second order PD differential operator, $\mathbf{g}_{2}(\xi)$, the nonlocal form of the second order derivatives can be evaluated as 


$$
\frac{\partial^{2} v_{i}}{\partial x_{j}^{2}}=\int_{H_{\mathbf{x}}}\left(v_{i}\left(\mathbf{x}^{\prime}\right)-v_{i}(\mathbf{x})\right) g_{2}^{(j, j)}(\xi) \mathrm{d} V^{\prime}
$$

where $i, j=1,2,3$. After substituting Eq.(29) into Eq. (28), the non-local form of Laplacian becomes

$$
\Delta \mathbf{v}=\int_{H_{\mathbf{x}}}\left[\begin{array}{l}
\left(v_{1}\left(\mathbf{x}^{\prime}\right)-v_{1}(\mathbf{x})\right)\left(\sum_{j=1}^{3} g_{2}^{(j, j)}(\xi)\right) \\
\left.\left(\mathbf{x}^{\prime}\right)-v_{2}(\mathbf{x})\right)\left(\sum_{j=1}^{3} g_{2}^{(j, j)}(\xi)\right) \\
\left(v_{3}\left(\mathbf{x}^{\prime}\right)-v_{3}(\mathbf{x})\right)\left(\sum_{j=1}^{3} g_{2}^{(j, j)}(\xi)\right)
\end{array}\right] \mathrm{d} V^{\prime}
$$

By considering the operator matrix provided in Eq. (17b), the non-local form of Laplacian operator in Eq. (30) can be expressed in compact form as

$$
\Delta \mathbf{v}(\mathbf{x})=\int_{H_{\mathbf{x}}} \operatorname{tr}\left(\mathbf{g}_{2}(\xi)\right)\left(\mathbf{v}\left(\mathbf{x}^{\prime}\right)-\mathbf{v}(\mathbf{x})\right) \mathrm{d} V^{\prime}
$$

By using Eq. (24), the transpose of the velocity gradient is defined as

$$
(\nabla \otimes \mathbf{v})^{T}=\left[\begin{array}{lll}
\frac{\partial v_{1}}{\partial x_{1}} & \frac{\partial v_{2}}{\partial x_{1}} & \frac{\partial v_{3}}{\partial x_{1}} \\
\frac{\partial v_{1}}{\partial x_{2}} & \frac{\partial v_{2}}{\partial x_{2}} & \frac{\partial v_{3}}{\partial x_{2}} \\
\frac{\partial v_{1}}{\partial x_{3}} & \frac{\partial v_{2}}{\partial x_{3}} & \frac{\partial v_{3}}{\partial x_{3}}
\end{array}\right]
$$

Therefore, the divergence of the transpose of the velocity gradient is

$$
\nabla \cdot(\nabla \otimes \mathbf{v})^{T}=\left[\begin{array}{l}
\frac{\partial^{2} v_{1}}{\partial x_{1}^{2}}+\frac{\partial^{2} v_{2}}{\partial x_{1} \partial x_{2}}+\frac{\partial^{2} v_{3}}{\partial x_{1} \partial x_{3}} \\
\frac{\partial^{2} v_{1}}{\partial x_{1} \partial x_{2}}+\frac{\partial^{2} v_{2}}{\partial x_{2}^{2}}+\frac{\partial^{2} v_{3}}{\partial x_{2} \partial x_{3}} \\
\frac{\partial^{2} v_{1}}{\partial x_{1} \partial x_{3}}+\frac{\partial^{2} v_{2}}{\partial x_{2} \partial x_{3}}+\frac{\partial^{2} v_{3}}{\partial x_{3}^{2}}
\end{array}\right]
$$

Subsequently, the non-local form derivatives in Eq. (33) can be expressed as

$$
\frac{\partial^{2} v_{i}}{\partial x_{j} \partial x_{k}}=\int_{H_{\mathbf{x}}}\left(v_{i}\left(\mathbf{x}^{\prime}\right)-v_{i}(\mathbf{x})\right) g_{2}^{(j, k)}(\xi) \mathrm{d} V^{\prime}
$$

where $i, j, k=1,2,3$. Eq.(33) can be converted into its non-local form as 


$$
\begin{aligned}
& \nabla \cdot(\nabla \otimes \mathbf{v})^{T}= \\
& \int_{H_{\mathbf{x}}}\left[\begin{array}{l}
\left(v_{1}\left(\mathbf{x}^{\prime}\right)-v_{1}(\mathbf{x})\right) g_{2}^{(1,1)}(\xi)+\left(v_{2}\left(\mathbf{x}^{\prime}\right)-v_{2}(\mathbf{x})\right) g_{2}^{(1,2)}(\xi)+\left(v_{3}\left(\mathbf{x}^{\prime}\right)-v_{3}(\mathbf{x})\right) g_{2}^{(1,3)}(\xi) \\
\left(v_{1}\left(\mathbf{x}^{\prime}\right)-v_{1}(\mathbf{x})\right) g_{2}^{(2,1)}(\xi)+\left(v_{2}\left(\mathbf{x}^{\prime}\right)-v_{2}(\mathbf{x})\right) g_{2}^{(2,2)}(\xi)+\left(v_{3}\left(\mathbf{x}^{\prime}\right)-v_{3}(\mathbf{x})\right) g_{2}^{(2,3)}(\xi) \\
\left(v_{1}\left(\mathbf{x}^{\prime}\right)-v_{1}(\mathbf{x})\right) g_{2}^{(3,1)}(\xi)+\left(v_{2}\left(\mathbf{x}^{\prime}\right)-v_{2}(\mathbf{x})\right) g_{2}^{(3,2)}(\xi)+\left(v_{3}\left(\mathbf{x}^{\prime}\right)-v_{3}(\mathbf{x})\right) g_{2}^{(3,3)}(\xi)
\end{array}\right]
\end{aligned}
$$

Eq.(35) can be expressed in a compact form as

$$
\nabla \cdot(\nabla \otimes \mathbf{v}(\mathbf{x}))^{T}=\int_{H_{\mathbf{x}}} \mathbf{g}_{2}(\xi) \cdot\left(\mathbf{v}\left(\mathbf{x}^{\prime}\right)-\mathbf{v}(\mathbf{x})\right) \mathrm{d} V^{\prime}
$$

In conclusion, the comparison of the local form and the non-local form of the velocity derivatives is summarized as

Table 1 Comparison of local and non-local velocity derivatives

\begin{tabular}{ccc}
\hline Velocity derivatives & Local Form & Non-local form \\
\hline$\nabla \cdot \mathbf{v}(\mathbf{x})$ & $\sum_{i=1}^{3} \frac{\partial v_{i}(\mathbf{x})}{\partial x_{i}}$ & $\int_{H_{\mathbf{x}}} \mathbf{g}_{1}(\xi) \cdot\left(\mathbf{v}\left(\mathbf{x}^{\prime}\right)-\mathbf{v}(\mathbf{x})\right) \mathrm{d} V^{\prime}$ \\
$\nabla \otimes \mathbf{v}(\mathbf{x})$ & $\frac{\partial v_{i}(\mathbf{x})}{\partial x_{j}} \mathbf{e}_{i} \otimes \mathbf{e}_{j}, i, j=1,2,3$ & $\int_{H_{\mathbf{x}}}\left(\mathbf{v}\left(\mathbf{x}^{\prime}\right)-\mathbf{v}(\mathbf{x})\right) \otimes \mathbf{g}_{1}(\xi) \mathrm{d} V^{\prime}$ \\
$\Delta \mathbf{v}(\mathbf{x})$ & $\sum_{j=1}^{3} \frac{\partial^{2} v_{i}(\mathbf{x})}{\partial x_{j}^{2}} \mathbf{e}_{i}, i=1,2,3$ & $\int_{H_{\mathbf{x}}} \operatorname{tr}\left(\mathbf{g}_{2}(\xi)\right)\left(\mathbf{v}\left(\mathbf{x}^{\prime}\right)-\mathbf{v}(\mathbf{x})\right) \mathrm{d} V^{\prime}$ \\
$\nabla \cdot(\nabla \otimes \mathbf{v}(\mathbf{x}))^{T}$ & $\sum_{j=1}^{3} \frac{\partial^{2} v_{j}(\mathbf{x})}{\partial x_{i} \partial x_{j}} \mathbf{e}_{i}, i=1,2,3$ & $\int_{H_{\mathbf{x}}} \mathbf{g}_{2}(\xi) \cdot\left(\mathbf{v}\left(\mathbf{x}^{\prime}\right)-\mathbf{v}(\mathbf{x})\right) \mathrm{d} V^{\prime}$ \\
\hline
\end{tabular}

In this study, non-local operators both for the first and the second order derivatives are provided. The non-local operators are derived by using second order Taylor series expansion. If only the first order derivative is considered and first order Taylor series expansion is used, the non-local operators for the first order derivatives, i.e. non-local gradient operator and nonlocal divergence operator, have the similar form as the ones from ( $\mathrm{Tu}$ and $\mathrm{Li}, 2017)$. However, in the present study, the second order Taylor series expansion is adopted and PD differential operator functions are directly determined by making them orthogonal to each term in the Taylor series expansion (Madenci et al., 2016). ). Therefore, the second order terms have effects on the first order operator due to the adoption of the orthogonal function properties. As a result, the formulations of the first order operators, i.e. non-local gradient operator and divergence operator, becomes different from the ones in ( $\mathrm{Tu}$ and $\mathrm{Li}, 2017)$. 


\section{A Non-local form of Navier-Stokes equations}

In this section, the non-local form of Navier-Stokes equations which describe the Newtonian fluid laminar flow is derived by using the non-local operator developed in Section 3.

\subsection{Conservation of Mass}

In classical fluid mechanics, the equation that describes the conservation of mass is

$$
\frac{\mathrm{D} \rho}{\mathrm{D} t}=-\rho \nabla \cdot \mathbf{v}
$$

where $\rho$ is the fluid density. The non-local form of velocity divergence operator in Table 1 is adopted to convert Eq. (37) into its non-local form as

$$
\frac{\mathrm{D} \rho}{\mathrm{D} t}=-\rho \int_{H_{\mathbf{x}}} \mathbf{g}_{1}(\xi) \cdot\left(\mathbf{v}\left(\mathbf{x}^{\prime}\right)-\mathbf{v}(\mathbf{x})\right) \mathrm{d} V^{\prime}
$$

\subsection{Constitutive Equations}

The stress is defined as

$$
\boldsymbol{\sigma}=-p \mathbf{I}+2 \mu \dot{\boldsymbol{\varepsilon}}
$$

where $p$ is the hydrostatic pressure, $\mathbf{I}$ is the second order unit tensor, $\mu$ is the dynamic viscosity, and $\dot{\boldsymbol{\varepsilon}}$ is the shear strain rate. For incompressible fluid and compressible fluid flow, the expressions for stress are different. Therefore, the constitutive equations are discussed separately.

\subsubsection{Incompressible fluid}

The shear strain rate of the incompressible fluid is defined as

$$
\dot{\boldsymbol{\varepsilon}}=\frac{1}{2}\left[\nabla \otimes \mathbf{v}+(\nabla \otimes \mathbf{v})^{T}\right]
$$

By using the non-local operator in Table 1, the non-local form of the shear strain rate is

$$
\dot{\boldsymbol{\varepsilon}}=\frac{1}{2} \int_{H_{\mathbf{x}}}\left[\left(\mathbf{v}\left(\mathbf{x}^{\prime}\right)-\mathbf{v}(\mathbf{x})\right) \otimes \mathbf{g}_{1}(\xi)+\mathbf{g}_{1}(\xi)^{T} \otimes\left(\mathbf{v}\left(\mathbf{x}^{\prime}\right)-\mathbf{v}(\mathbf{x})\right)^{T}\right] \mathrm{d} V^{\prime}
$$

As a result, the stress definition in Eq. (39) can be expressed as

$$
\boldsymbol{\sigma}=-p \mathbf{I}+\mu \int_{H_{\mathbf{x}}}\left[\left(\mathbf{v}\left(\mathbf{x}^{\prime}\right)-\mathbf{v}(\mathbf{x})\right) \otimes \mathbf{g}_{1}(\xi)+\mathbf{g}_{1}(\xi)^{T} \otimes\left(\mathbf{v}\left(\mathbf{x}^{\prime}\right)-\mathbf{v}(\mathbf{x})\right)^{T}\right] \mathrm{d} V^{\prime}
$$

Regarding the incompressible fluid flow, a prohibitively small time step size is required for stability in the pressure simulation (Morris et al., 1997). Therefore, artificial compressibility methods (Drikakis and Rider, 2005) are introduced which makes the incompressible fluid weakly compressible. The Tait equation of state is adopted to model such flows, in which the pressure is an explicit function of local fluid density as (Batchelor, 2000) 


$$
p=B\left(\left(\frac{\rho_{t}}{\rho_{0}}\right)^{\gamma}-1\right)
$$

where $\rho_{t}$ represents the current local density, $\rho_{0}$ represents the initial density and $\gamma$ represents the material constant which is $\gamma=7$ for water.

In Eq. (43) $B$ is a parameter which can be defined as (Monaghan, 1994)

$$
B=\frac{\rho_{0} c^{2}}{\gamma}
$$

where $c$ represents the speed of sound. The speed of sound can be approximated as (Becker and Teschner, 2007)

$$
c=\left|\mathbf{v}_{f}\right| / \sqrt{\eta}
$$

with $\left|\mathbf{v}_{f}\right|$ representing the maximum magnitude of fluid flow velocity. The term $\eta$ represents the density variation with a typical value being 0.01 , indicating that density varies at most $1 \%$.

\subsubsection{Compressible fluid}

The shear strain rate for compressible fluid is defined as

$$
\dot{\boldsymbol{\varepsilon}}=\frac{1}{2}\left[\nabla \otimes \mathbf{v}+(\nabla \otimes \mathbf{v})^{T}\right]-\frac{1}{3}(\nabla \cdot \mathbf{v}) \mathbf{I}
$$

By considering the non-local velocity operator in Table 1 and Eq. (41), the non-local form of the shear strain rate can be expressed as

$$
\begin{aligned}
\dot{\boldsymbol{\varepsilon}} & =\frac{1}{2} \int_{H_{\mathbf{x}}}\left[\left(\mathbf{v}\left(\mathbf{x}^{\prime}\right)-\mathbf{v}(\mathbf{x})\right) \otimes \mathbf{g}_{1}(\xi)+\mathbf{g}_{1}(\xi)^{T} \otimes\left(\mathbf{v}\left(\mathbf{x}^{\prime}\right)-\mathbf{v}(\mathbf{x})\right)^{T}\right] \mathrm{d} V^{\prime} \\
& -\frac{1}{3} \int_{H_{\mathbf{x}}}\left[\left(\mathbf{g}_{1}(\xi) \cdot\left(\mathbf{v}\left(\mathbf{x}^{\prime}\right)-\mathbf{v}(\mathbf{x})\right)\right) \mathbf{I}\right] \mathrm{d} V^{\prime}
\end{aligned}
$$

As a result, the stress defined in Eq. (39) can be expressed as

$$
\begin{aligned}
\boldsymbol{\sigma} & =-p \mathbf{I}+\mu \int_{H_{\mathbf{x}}}\left[\left(\mathbf{v}\left(\mathbf{x}^{\prime}\right)-\mathbf{v}(\mathbf{x})\right) \otimes \mathbf{g}_{1}(\xi)+\mathbf{g}_{1}(\xi)^{T} \otimes\left(\mathbf{v}\left(\mathbf{x}^{\prime}\right)-\mathbf{v}(\mathbf{x})\right)^{T}\right] \mathrm{d} V^{\prime} \\
& -\frac{2 \mu}{3} \int_{H_{\mathbf{x}}}\left[\left(\mathbf{g}_{1}(\xi) \cdot\left(\mathbf{v}\left(\mathbf{x}^{\prime}\right)-\mathbf{v}(\mathbf{x})\right)\right) \mathbf{I}\right] \mathrm{d} V^{\prime}
\end{aligned}
$$

Regarding compressible fluid flow the material derivative of pressure is ( $\mathrm{Tu}$ and $\mathrm{Li}, 2017$ )

$$
\frac{\mathrm{D} p}{\mathrm{D} t}=-K_{f} \nabla \cdot \mathbf{v}
$$

where $K_{f}$ is the elastic bulk modulus of fluid. 
The non-local velocity divergence operator in Table 1 is adopted to convert Eq.(49) into its non-local form as

$$
\frac{\mathrm{D} p}{\mathrm{D} t}=-K_{f} \int_{H_{\mathbf{x}}}\left(\mathbf{g}_{1}(\xi) \cdot\left(\mathbf{v}\left(\mathbf{x}^{\prime}\right)-\mathbf{v}(\mathbf{x})\right)\right) \mathrm{d} V^{\prime}
$$

\subsection{Conservation of Momentum}

The local form of the equation of motion is

$$
\rho \frac{\mathrm{D} \mathbf{v}}{\mathrm{D} t}=\nabla \cdot \boldsymbol{\sigma}+\rho \mathbf{b}
$$

where $\mathbf{b}$ represents the body force.

By using the definitions in Eq. (39) into Eq. (51), the equation of motion can be expressed in terms of pressure and shear strain rate as

$$
\rho \frac{D \mathbf{v}}{D t}=\nabla \cdot(-p \mathbf{I}+2 \mu \dot{\boldsymbol{\varepsilon}})+\rho \mathbf{b}
$$

By applying the Leibniz rule Eq. (52) can be written as

$$
\rho \frac{D \mathbf{v}}{D t}=-\nabla \cdot(p \mathbf{I})+2 \mu(\nabla \cdot \dot{\boldsymbol{\varepsilon}})+\dot{\boldsymbol{\varepsilon}} \nabla \cdot(2 \mu)+\rho \mathbf{b}
$$

In this study, the dynamic viscosity $\mu$ is assumed to be constant, therefore Eq. (53) takes the form as

$$
\rho \frac{D \mathbf{v}}{D t}=-\nabla \cdot(p \mathbf{I})+2 \mu(\nabla \cdot \dot{\boldsymbol{\varepsilon}})+\rho \mathbf{b}
$$

Similar to the non-local velocity operators in Table 1, the non-local form of the first term on the right-hand side of Eq. (54) can be expressed as

$$
\nabla \cdot(p \mathbf{I})=\int_{H_{\mathbf{x}}}\left[\left(p\left(\mathbf{x}^{\prime}\right)-p(\mathbf{x})\right) \mathbf{g}_{1}(\xi)\right] \mathrm{d} V^{\prime}
$$

\subsubsection{Incompressible fluid}

By considering the shear strain rate expression provided in Eq.(40) for an incompressible fluid, the divergence of the shear strain rate can be expressed as

$$
\nabla \cdot \dot{\boldsymbol{\varepsilon}}=\frac{1}{2} \nabla \cdot\left[\nabla \otimes \mathbf{v}+(\nabla \otimes \mathbf{v})^{T}\right]
$$

Eq. (56) can also be presented as

$$
\nabla \cdot \dot{\boldsymbol{\varepsilon}}=\frac{1}{2}\left(\Delta \mathbf{v}+\nabla \cdot(\nabla \otimes \mathbf{v})^{T}\right)
$$


By applying the non-local second order operators provided in Table 1, the non-local form of divergence of the shear strain rate can be obtained as

$$
\nabla \cdot \dot{\boldsymbol{\varepsilon}}=\frac{1}{2} \int_{H_{\mathbf{x}}}\left[\operatorname{tr}\left(\mathbf{g}_{2}(\xi)\right)\left(\mathbf{v}\left(\mathbf{x}^{\prime}\right)-\mathbf{v}(\mathbf{x})\right)+\mathbf{g}_{2}(\xi) \cdot\left(\mathbf{v}\left(\mathbf{x}^{\prime}\right)-\mathbf{v}(\mathbf{x})\right)\right] \mathrm{d} V^{\prime}
$$

By substituting Eq.(55) and Eq.(58) into Eq.(54), the non-local form of the equation of motion for an incompressible fluid can be obtained as

$$
\begin{aligned}
\rho \frac{D \mathbf{v}}{D t} & =\int_{H_{\mathbf{x}}}\left\{\mu\left[\operatorname{tr}\left(\mathbf{g}_{2}(\xi)\right)\left(\mathbf{v}\left(\mathbf{x}^{\prime}\right)-\mathbf{v}(\mathbf{x})\right)+\mathbf{g}_{2}(\xi) \cdot\left(\mathbf{v}\left(\mathbf{x}^{\prime}\right)-\mathbf{v}(\mathbf{x})\right)\right]\right\} \mathrm{d} V^{\prime} \\
& -\int_{H_{\mathbf{x}}}\left\{\left(p\left(\mathbf{x}^{\prime}\right)-p(\mathbf{x})\right) \mathbf{g}_{1}(\xi)\right\} \mathrm{d} V^{\prime}+\rho \mathbf{b}
\end{aligned}
$$

\subsubsection{Compressible fluid}

By considering the shear strain rate expression provided in Eq.(46) for compressible fluid, the divergence of the shear strain rate can be expressed as

$$
\nabla \cdot \dot{\boldsymbol{\varepsilon}}=\frac{1}{2}\left(\Delta \mathbf{v}+\nabla \cdot(\nabla \otimes \mathbf{v})^{T}\right)-\frac{1}{3} \nabla \cdot((\nabla \cdot \mathbf{v}) \mathbf{I})
$$

It can be proved that $\nabla \cdot((\nabla \cdot \mathbf{v}) \mathbf{I})=\nabla \cdot(\nabla \otimes \mathbf{v})^{T}$. By considering Eq. (58) and Table 1, the non-local form of the divergence of shear strain rate for compressible fluids can be obtained as

$$
\begin{aligned}
\nabla \cdot \dot{\boldsymbol{\varepsilon}}= & \int_{H_{\mathbf{x}}}\left\{\frac{1}{2}\left[\operatorname{tr}\left(\mathbf{g}_{2}(\xi)\right)\left(\mathbf{v}\left(\mathbf{x}^{\prime}\right)-\mathbf{v}(\mathbf{x})\right)+\mathbf{g}_{2}(\xi) \cdot\left(\mathbf{v}\left(\mathbf{x}^{\prime}\right)-\mathbf{v}(\mathbf{x})\right)\right]\right\} \mathrm{d} V^{\prime} \\
& -\int_{H_{\mathbf{x}}}\left\{\frac{1}{3}\left(\mathbf{g}_{2}(\xi) \cdot\left(\mathbf{v}\left(\mathbf{x}^{\prime}\right)-\mathbf{v}(\mathbf{x})\right)\right)\right\} \mathrm{d} V^{\prime}
\end{aligned}
$$

By substituting Eq.(55) and Eq. (61) into Eq. (54), the non-local form of the equation of motion for a compressible fluid can be obtained as

$$
\rho \frac{D \mathbf{v}}{D t}=\int_{H_{\mathbf{x}}}\left\{\begin{array}{l}
\mu\left[\operatorname{tr}\left(\mathbf{g}_{2}(\xi)\right)\left(\mathbf{v}\left(\mathbf{x}^{\prime}\right)-\mathbf{v}(\mathbf{x})\right)+\mathbf{g}_{2}(\xi) \cdot\left(\mathbf{v}\left(\mathbf{x}^{\prime}\right)-\mathbf{v}(\mathbf{x})\right)\right] \\
-\frac{2}{3} \mu\left[\mathbf{g}_{2}(\xi) \cdot\left(\mathbf{v}\left(\mathbf{x}^{\prime}\right)-\mathbf{v}(\mathbf{x})\right)\right]-\left(p\left(\mathbf{x}^{\prime}\right)-p(\mathbf{x})\right) \mathbf{g}_{1}(\xi)
\end{array}\right\} \mathrm{d} V^{\prime}+\rho \mathbf{b}
$$

As provided in Eq.(59) and (62), the conservation equation of momentum is directly expressed in terms of velocity and pressure, leading to the existence of the second order derivatives. Due to the adoption of the corresponding PD differential operator for the second order derivatives provided in Table 1 , the acceleration can be directly calculated with one integration with second order accuracy. The error introduced by the integration can be reduced compared to first order approximations where the integration needs to be performed twice to 
calculate the acceleration. In addition, the computational time can also be reduced by using integration only once.

\section{Numerical Implementation}

In this section, the numerical implementation for fluid flow simulation is provided. There are two types of Lagrangian descriptions, i.e. total Lagrangian description and updated Lagrangian description. Both Lagrangian approaches have been discussed in state-based peridynamics (Bergel and Li, 2016). Therefore, non-local Navier-Stokes equations are numerically implemented both in total and updated Lagrangian approaches.

\subsection{Kinematic Quantities}

The related kinematic quantities for numerical implementations are explained as follows. As shown in Fig. 3, point $i$ represents the point of interest and point $j$ represents one of its family members.

The definitions of relative kinematic quantities are shown in Fig. 3. The terms $\mathbf{x}, \mathbf{u}$, and $\mathbf{v}$ represent position, displacement, and velocity vectors, respectively. The subscript (0) represents the initial configuration. On the other hand the subscript $(n)$ represents the current configuration. For example, $\mathbf{x}_{0}^{i}$ and $\mathbf{v}_{0}^{i}$ represent the position and the velocity vector for point $i$ in the initial configuration, respectively. Here, $\xi_{0}, \xi_{n}, \xi_{n+1}$ represent the relative position vectors in the initial, current, and updated configurations, respectively.

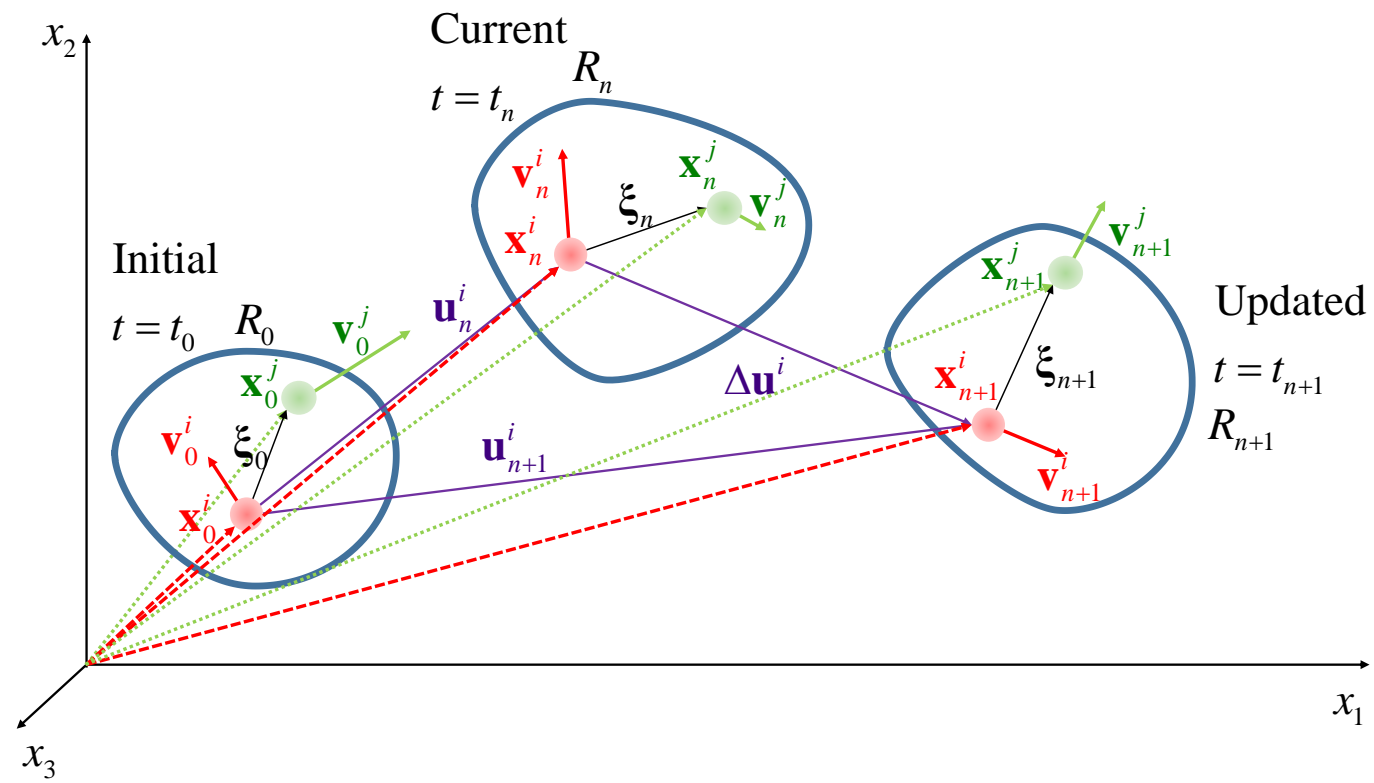

Fig. 3 Initial, current, and updated configurations in the fluid domain, $R$ 


\subsection{Total Lagrangian Method}

For numerical implementation, the integration is performed by using the discrete particles. Based on the derivations in Section 4, the algorithm in a total Lagrangian description is presented.

\subsubsection{Governing equations in total Lagrangian description}

In the total Lagrangian description, the governing equations in discretized form for incompressible fluid flows and compressible fluid flows are summarized as follows.

Conservation of Mass:

The discretized form of Eq. (38) can be evaluated as

$$
\rho_{n+1}^{i}=\rho_{n}^{i}-\rho_{n}^{i} \Delta t \sum_{j=1}^{N_{i}}\left(\mathbf{g}_{1}\left(\boldsymbol{\xi}_{0}\right) \cdot\left(\mathbf{v}_{n}^{j}-\mathbf{v}_{n}^{i}\right)\right) V_{0}^{j}
$$

where $n$ represents the time step number, $\Delta t$ represents the time step size , $N_{i}$ represents the total number of family members of point $i$ and $V_{0}^{j}$ represents the initial volume of point $j$. It should be noted that the density remains constant for incompressible fluid flow that is $\rho_{n+1}^{i}=\rho_{0}^{i}$.

Constitutive Equations for Pressure:

For incompressible fluid flow, the discretized form of Eq. (43) can be evaluated as

$$
p_{n+1}^{i}=B\left(\left(\frac{\rho_{n+1}^{i}}{\rho_{0}^{i}}\right)^{\gamma}-1\right)
$$

For compressible fluid flow, the discretized form of Eq. (50) can be evaluated as

$$
p_{n+1}^{i}=p_{n}^{i}-K_{f} \Delta t \sum_{j=1}^{N_{i}}\left(\mathbf{g}_{1}\left(\boldsymbol{\xi}_{0}\right) \cdot\left(\mathbf{v}_{n}^{j}-\mathbf{v}_{n}^{i}\right)\right) V_{0}^{j}
$$

\section{Conservation of Momentum:}

For incompressible fluid flow, the discretized form of Eq. (59) can be evaluated as

$$
\begin{aligned}
\mathbf{a}_{n+1}^{i} & =\frac{1}{\rho_{n+1}^{i}} \sum_{j=1}^{N_{i}}\left[\mu\left(\operatorname{tr}\left(\mathbf{g}_{2}\left(\xi_{0}\right)\right)\left(\mathbf{v}_{n}^{j}-\mathbf{v}_{n}^{i}\right)+\mathbf{g}_{2}\left(\xi_{0}\right) \cdot\left(\mathbf{v}_{n}^{j}-\mathbf{v}_{n}^{i}\right)\right)-\left(p_{n}^{j}-p_{n}^{i}\right) \mathbf{g}_{1}\left(\xi_{0}\right)\right] V_{0}^{j} \\
& +\mathbf{b}_{n+1}^{i}
\end{aligned}
$$

where $\mathbf{a}^{i}$ represents the acceleration of point $i$.

For compressible fluid flow, the discretized form of Eq. (62) can be evaluated as 


$$
\mathbf{a}_{n+1}^{i}=\frac{1}{\rho_{n+1}^{i}} \sum_{j=1}^{N_{i}}\left[\begin{array}{c}
\mu\left(\operatorname{tr}\left(\mathbf{g}_{2}\left(\xi_{0}\right)\right)\left(\mathbf{v}_{n}^{j}-\mathbf{v}_{n}^{i}\right)+\mathbf{g}_{2}\left(\xi_{0}\right) \cdot\left(\mathbf{v}_{n}^{j}-\mathbf{v}_{n}^{i}\right)\right) \\
-\frac{2}{3} \mu\left(\mathbf{g}_{2}\left(\xi_{0}\right) \cdot\left(\mathbf{v}_{n}^{j}-\mathbf{v}_{n}^{i}\right)\right)-\left(p_{n}^{j}-p_{n}^{i}\right) \mathbf{g}_{1}\left(\xi_{0}\right)
\end{array}\right] V_{0}^{j}+\mathbf{b}_{n+1}^{i}
$$

The Velocity Verlet algorithm (Swope et al., 1982) is used for updating the velocity and displacement fields as

$$
\begin{gathered}
\mathbf{v}_{n+1}^{i}=\mathbf{v}_{n}^{i}+\frac{1}{2}\left(\mathbf{a}_{n+1}^{i}+\mathbf{a}_{n}^{i}\right) \Delta t \\
\mathbf{x}_{n+1}^{i}=\mathbf{x}_{n}^{i}+\mathbf{v}_{n}^{i} \Delta t+\frac{1}{2} \mathbf{a}_{n}^{i} \Delta t^{2}
\end{gathered}
$$

\subsubsection{Numerical algorithm for total Lagrangian description}

The numerical algorithm for total Lagrangian approach is provided in Fig. 4. Fig. 5(a) represents the PD differential operator construction, and Fig. 5(b) represents the boundary implementation. Since the total Lagrangian approach is adopted, the family members of each point remain the same during the time integration. The PD differential operator is constructed based on the initial configuration, i.e. peridynamic operator, $\mathbf{g}$ is a function of initial relative positions $\mathbf{g}\left(\xi_{0}\right)$. Therefore, the PD differential operator is constructed prior to the time integration, as shown in Fig. 4. Therefore, during the numerical simulations, the PD differential operator associated with each PD bond is stored in a time-independent array. In addition, for PD differential operator construction, Math Kernel Library (MKL) (Intel, 2011) is utilized. The function 'DGESV' (Anderson et al., 1990) which uses LU decomposition with partial pivoting and row interchanges is utilized for solving the coefficient matrix , a , in Eq.(A.11). 


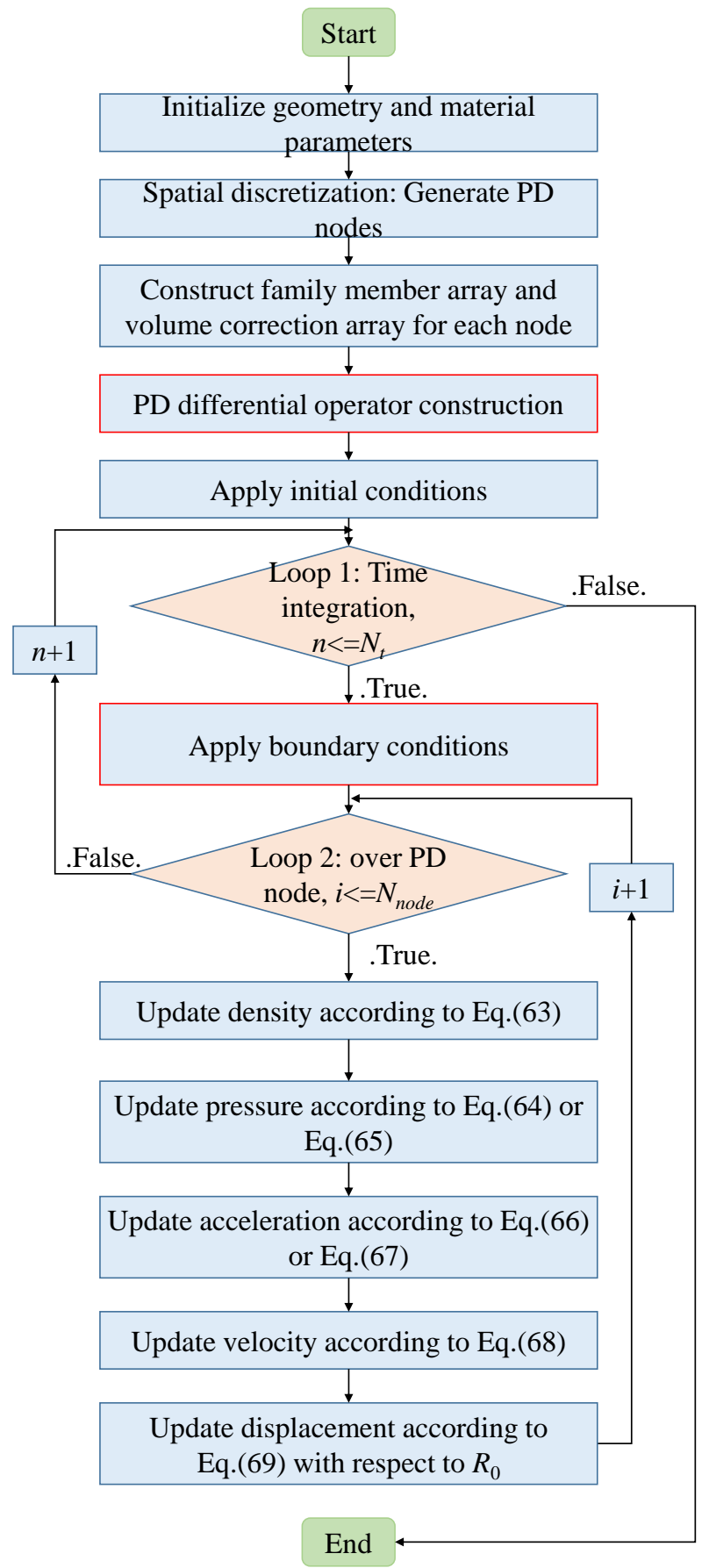

Fig. 4 Flowchart of the numerical algorithm for total Lagrangian approach 
PD differential operator construction

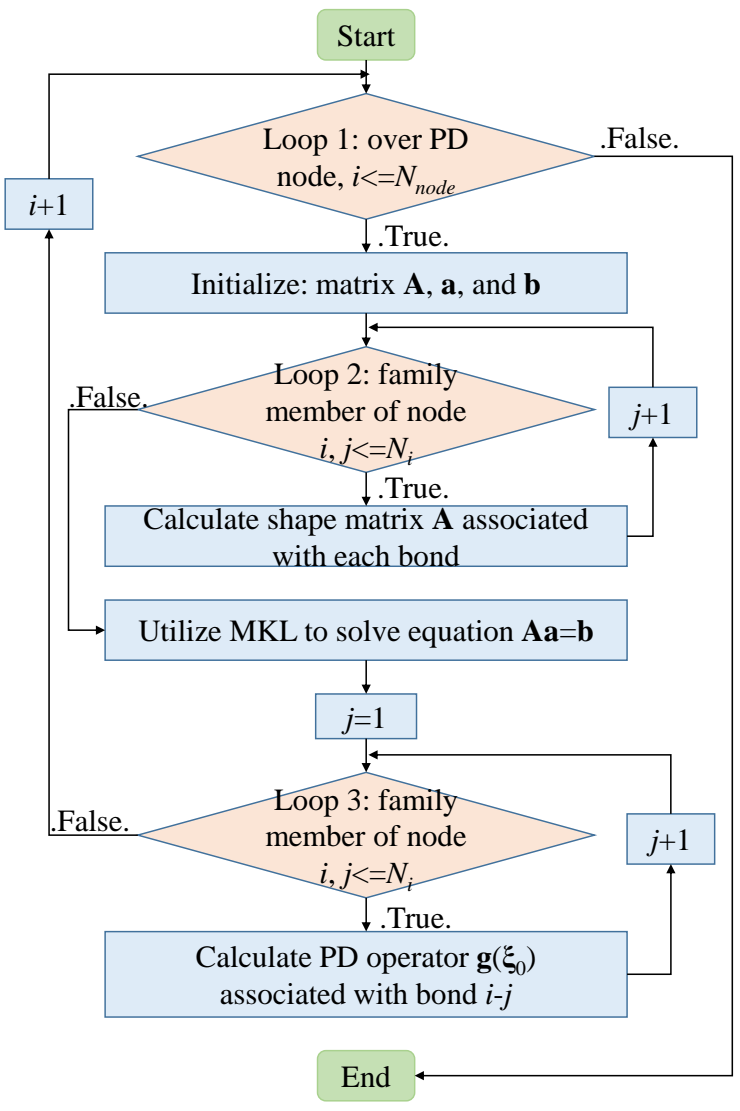

(a)
Apply boundary conditions

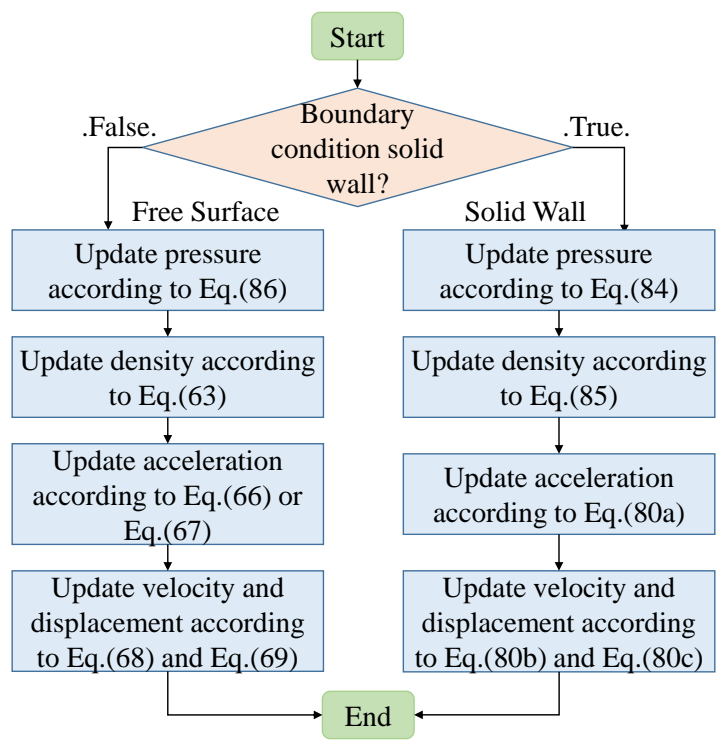

(b)

Fig. 5 Flowchart for: (a) PD differential operator construction, and (b) boundary implementation

\subsection{Updated Lagrangian Method}

In this section, the numerical algorithm for an updated Lagrangian description is developed for fluid flow simulations using the PD differential operator.

\subsubsection{Updated Lagrangian equations}

The non-local fluid flow governing equations in the total Lagrangian description is already provided in Section 5.2.1. In the updated Lagrangian approach, the initial volume of point $j$, $V_{0}^{j}$, will be updated by using the current volume, $V_{n}^{j}$ as

$$
V_{n}^{j}=J_{n} V_{0}^{j}
$$

where $J_{n}$ is the determinant of Jacobian which is defined as

$$
J_{n}=\operatorname{det}\left(\mathbf{F}_{n}\right)
$$

In Eq.(71), $\mathbf{F}_{n}$ is the deformation gradients with respect to initial configuration, which is defined as 


$$
\mathbf{F}_{n}=\frac{\partial \mathbf{x}_{n}}{\partial \mathbf{x}_{0}}
$$

Similarly, the volume in the updated configuration $V_{n+1}^{j}$ can be expressed in terms of initial volume as $\mathrm{d} V_{n+1}^{j}=J_{n+1} \mathrm{~d} V_{0}^{j}$ with $J_{n+1}=\operatorname{det}\left(\mathbf{F}_{n+1}\right)$ and $\mathbf{F}_{n+1}=\partial \mathbf{x}_{n+1} / \partial \mathbf{x}_{0}$.

The volume in the updated configuration is related to the current configuration as

$$
\mathrm{d} V_{n+1}^{j}=J_{n+1} J_{n}^{-1} \mathrm{~d} V_{n}^{j}=\operatorname{det}(\Delta \mathbf{F}) \mathrm{d} V_{n}^{j}
$$

where the relative deformation gradient $\Delta \mathbf{F}$ is defined as

$$
\Delta \mathbf{F}=\frac{\partial \mathbf{x}_{n+1}}{\partial \mathbf{x}_{n}}=\frac{\partial \mathbf{x}_{n+1}}{\partial \mathbf{x}} / \frac{\partial \mathbf{x}_{n}}{\partial \mathbf{x}}=\mathbf{F}_{n+1} \mathbf{F}_{n}^{-1}
$$

On the other hand, the non-local form of the deformation gradient tensor is defined in (Madenci, 2017) by using the PD differential operator as

$$
\mathbf{F}=\frac{\operatorname{tr}(\mathbf{I})}{m} \int_{H_{\mathbf{x}}} w_{F}\left(\mathbf{y}^{\prime}-\mathbf{y}\right) \otimes\left(\mathbf{x}^{\prime}-\mathbf{x}\right) \mathrm{d} V^{\prime}
$$

with $w_{F}=\left(\delta /\left|\mathbf{x}^{\prime}-\mathbf{x}\right|\right)^{2}$. The parameter $\mathbf{I}$ is the identity matrix with its trace $\operatorname{tr}(\mathbf{I})$ being 2 or 3 depending on the dimensions of the analysis. The parameter $m$ is evaluated as (Madenci, 2017)

$$
m=V_{H} \delta^{2}
$$

where $V_{H}$ represents the volume of a sphere, $V_{H}=4 \pi \delta^{3} / 3$ for $3 D$ and volume of a disk, $V_{H}=h \pi \delta^{2}$ for $2 D$ with $h$ being the thickness of a disk (Madenci, 2017). Therefore, the nonlocal form of deformation gradient of point $i$ for current and updated configurations are defined as (Madenci, 2017; Silling et al., 2007; Tu and Li, 2017)

$$
\mathbf{F}_{n}=\frac{\operatorname{tr}(\mathbf{I})}{m} \int_{H_{n}^{i}} w_{F}\left(\xi_{n}\right) \otimes\left(\xi_{n}\right) \mathrm{d} V_{n}^{j}
$$

and

$$
\mathbf{F}_{n+1}=\frac{\operatorname{tr}(\mathbf{I})}{m} \int_{H_{n}^{i}} w_{F}\left(\xi_{n+1}\right) \otimes\left(\xi_{n}\right) \mathrm{d} V_{n}^{j}
$$

By using the definition in Eq. (74), the non-local form of relative deformation gradient can be defined as

$$
\Delta \mathbf{F}=\left[\int_{H_{n}^{i}} w_{F}\left(\xi_{n+1}\right) \otimes\left(\xi_{n}\right) \mathrm{d} V_{n}^{j}\right]\left[\int_{H_{n}^{i}} w_{F}\left(\xi_{n}\right) \otimes\left(\xi_{n}\right) \mathrm{d} V_{n}^{j}\right]^{-1}
$$

Based on the developed non-local relative deformation gradient in Eq. (79), the volume for each material point is calculated by using Eq. (73). 


\subsubsection{Numerical algorithm for updated Lagrangian description}

The numerical algorithm for the updated Lagrangian approach is provided in Fig. 6. And Fig. 7 represents the calculation of relative deformation gradient. Within each time integration step, the coordinate of each node is updated as $\mathbf{x}_{n}=\mathbf{x}_{0}+\mathbf{u}_{n}$. Therefore, the family members of each point are updated in each time step. The PD differential operator construction process is the same as Fig. 5(a) except that the PD differential operator and the weighted function are constructed based on the current configuration, i.e. $\mathbf{g}$ and $w$ are functions of the relative position in the current configuration $\mathbf{g}\left(\xi_{n}\right)$ and $w\left(\left|\xi_{n}\right|\right)$. Consequently, the PD differential operator associated with each bond is updated simultaneously for each time step. Therefore, in terms of computational time, it could be inferred that the updated Lagrangian program is more time consuming than the total Lagrangian program. 


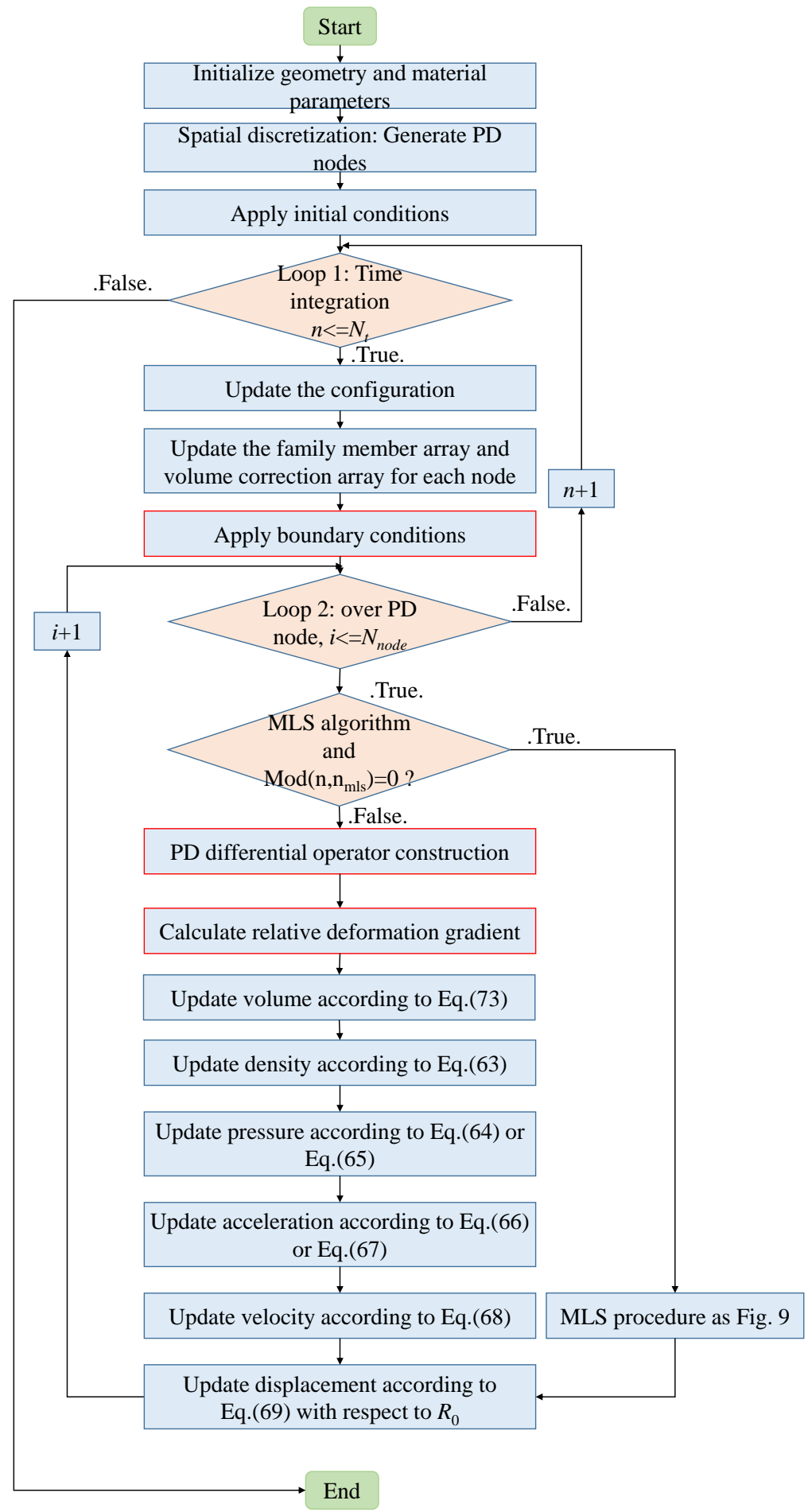

Fig. 6 Flowchart of the numerical algorithm for updated Lagrangian approach 


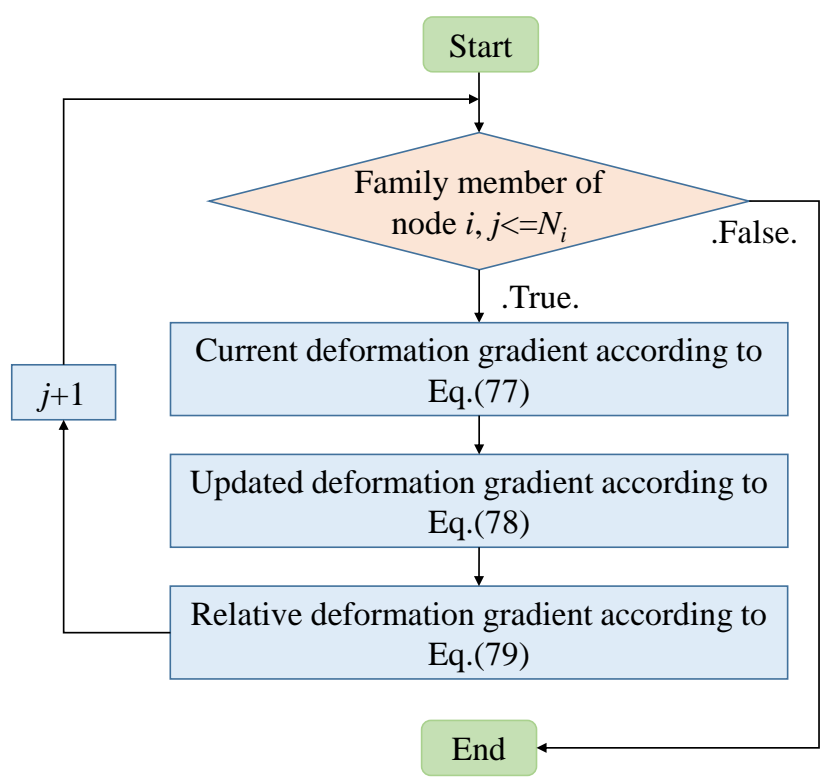

Fig. 7 Flowchart for relative deformation gradient calculation

\subsection{Numerical Treatments}

\subsubsection{Boundary treatment}

The implementation of a solid wall is a crucial issue in flow simulations. Fictitious layers (Madenci and Oterkus, 2014; Oterkus and Madenci, 2015, 2017; Oterkus et al., 2014) can be used to implement the boundary conditions. As illustrated in Fig. 8, the fictitious layers (shown by red spheres) are located outside the boundary to simulate the solid wall. The thickness of the fictitious layers is chosen as the size of the horizon (Macek and Silling, 2007). The positions of fictitious particles remain the same during the time integration (Adami et al., 2012; Cao et al., 2014). In Fig. 8, particle $i$ represents the fictitious particles. Particle $j$ represents one of its family member which belongs to fluid particles. The acceleration, velocity, and displacement of the fictitious particle $i$ are defined as the same as the solid wall, i.e.

$$
\begin{aligned}
\mathbf{a}_{i} & =\mathbf{a}_{\text {solid wall }} \\
\mathbf{v}_{i} & =\mathbf{v}_{\text {solid wall }} \\
\mathbf{u}_{i} & =\mathbf{u}_{\text {solid wall }}
\end{aligned}
$$

For the pressure evaluation of particle $i$, the formulation proposed by Adami et al. (Adami et al., 2012) is utilized. The viscous interaction between particle $i$ and $j$ is simply omitted (Adami et al., 2012). In order to eliminate the penetration of fluid particles into the wall surface, the force balance at the wall interface is enforced as (Adami et al., 2012) 


$$
\frac{\mathrm{d} \mathbf{v}_{j}}{\mathrm{~d} t}=-\frac{\nabla p_{j}}{\rho_{j}}+\mathbf{b}=\mathbf{a}_{i}
$$

where $\mathbf{a}_{i}$ represents the acceleration of the solid particle $i$ in the fictitious domain. The term $p_{j}$ is the pressure of the fluid particle and $\rho_{j}$ is the density of the fluid particle. According to (Adami et al., 2012), Eq. (81) can be further calculated as

$$
\int \nabla p \cdot \mathbf{d} l=\rho_{j} \int\left(\mathbf{b}-\mathbf{a}_{i}\right) \cdot \mathbf{d} l
$$

where $\mathbf{d} l$ is a vectorial length element between fluid and fictitious particles (Adami et al., 2012). For a single bond between the fluid and the fictitious particle, Eq.(82) takes the form as

$$
p_{i}-p_{j}=\rho_{j}\left(\mathbf{b}-\mathbf{a}_{i}\right) \cdot \xi_{i j}
$$

where $\xi_{i j}$ represents the relative position from the fluid particle to the dummy particle which can be expressed as $\xi_{i j}=\mathbf{x}_{i}-\mathbf{x}_{j}$, shown in Fig. 8. Consequently, the pressure of the fictitious particle $i, p_{i}$ can be obtained by summation of all contributions of neighbouring fluid particles using the weighted function as (Adami et al., 2012)

$$
p_{i}=\frac{\sum_{j=1}^{N_{i, f l u i d}}\left(p_{j}+\rho_{j}\left(\mathbf{b}-\mathbf{a}_{i}\right) \cdot \boldsymbol{\xi}_{i j}\right) w\left(\left|\xi_{i j}\right|\right)}{\sum_{j=1}^{N_{i, f l u i d}} w\left(\left|\xi_{i j}\right|\right)}
$$

where $N_{i, f u i d}$ represents the total number of the family member of particle $i$ which belongs to fluid particles. The weighted function $w\left(\left|\xi_{i j}\right|\right)$ is defined as in Eq.(12).

Then the density of the fictitious particle $i$ can be calculated by using Eq. (43) as

$$
\rho_{i}=\rho_{0}\left(\frac{p_{i}}{B}+1\right)^{1 / \gamma}
$$




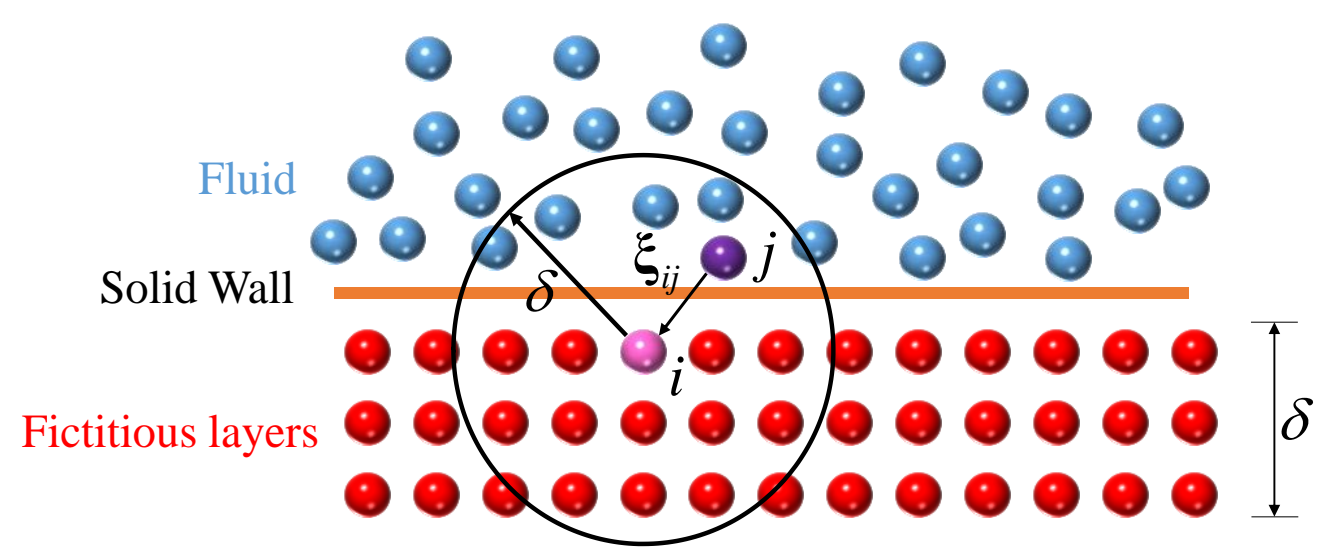

Fig. 8 Illustration of solid boundary implementation

Similarly, the free surface boundary conditions are implemented by using dummy particles. Fictitious layer with its thickness being equal to the size of the horizon is added on the free surfaces. The material properties of the dummy particles are chosen the same as the fluid particles. The acceleration, velocity, displacement, and density are calculated for both fluid and dummy particles in free surface. The only difference is that free surface pressure is applied to the fictitious layer as

$$
p_{\text {fictitious particles }}=p_{\text {free sufface }}=0
$$

\subsubsection{Free surface flow treatments}

In order to improve the numerical stability of free surface flow simulation, such as dam collapse simulation, extra numerical implementations are used. First, a damping time $t_{\text {damp }}$ is chosen during which the acceleration of each PD node is multiplied by a factor $\zeta(t)$ as (Adami et al., 2012)

$$
\zeta(t)=\left\{\begin{array}{cc}
0.5\left[\sin \left(\left(-0.5+t / t_{\text {damp }}\right) \pi\right)+1\right] & , t<t_{\text {damp }} \\
1 & , t \geq t_{\text {damp }}
\end{array}\right.
$$

Second, moving least squares (MLS) method (Colagrossi and Landrini, 2003) is adopted for every $n_{M L S}$ time step to smooth the velocity and density profiles as

$$
\begin{aligned}
\mathbf{v}\left(\mathbf{x}_{n}^{i}\right) & =\frac{\sum_{j} \mathbf{v}\left(\mathbf{x}_{n}^{j}\right) w_{M L S}\left(\mathbf{x}_{n}^{i}, \mathbf{x}_{n}^{j}\right)}{\sum_{j} w_{M L S}\left(\mathbf{x}_{n}^{i}, \mathbf{x}_{n}^{j}\right)} \\
\rho\left(\mathbf{x}_{n}^{i}\right) & =\sum_{j} m_{\text {mass }} w_{M L S}\left(\mathbf{x}_{n}^{i}, \mathbf{x}_{n}^{j}\right)
\end{aligned}
$$


with $m_{\text {mass }}$ representing the initial mass of one PD particle, $w_{M L S}$ is the smoothing weighted function which can be expressed as (Colagrossi and Landrini, 2003)

$$
w_{M L S}\left(\mathbf{x}_{n}^{i}, \mathbf{x}_{n}^{j}\right)=\left[\beta_{0}\left(\mathbf{x}_{n}^{i}\right)+\beta_{1}\left(\mathbf{x}_{n}^{i}\right)\left(-\xi_{n, x}\right)+\beta_{2}\left(\mathbf{x}_{n}^{i}\right)\left(-\xi_{n, y}\right)\right] w_{s}\left(\mathbf{x}_{n}^{i}, \mathbf{x}_{n}^{j}\right)
$$

with

$$
\begin{aligned}
& \xi_{n}=\xi_{n, x} \mathbf{e}_{1}+\xi_{n, y} \mathbf{e}_{2} \\
& \boldsymbol{\beta}\left(\mathbf{x}_{n}^{i}\right)=\left(\begin{array}{l}
\beta_{0} \\
\beta_{1} \\
\beta_{2}
\end{array}\right)=\mathbf{S}^{-1}\left(\mathbf{x}_{n}^{i}\right)\left[\begin{array}{l}
1 \\
0 \\
0
\end{array}\right] \\
& \mathbf{S}\left(\mathbf{x}_{n}^{i}\right)=\sum_{B} w\left(\mathbf{x}_{n}^{i}, \mathbf{x}_{n}^{j}\right) \tilde{\mathbf{S}}\left(\mathbf{x}_{n}^{i}, \mathbf{x}_{n}^{j}\right) \\
& \tilde{\mathbf{S}}\left(\mathbf{x}_{n}^{i}, \mathbf{x}_{n}^{j}\right)=\left[\begin{array}{ccc}
1 & -\xi_{n, x} & -\xi_{n, y} \\
-\xi_{n, x} & \left(\xi_{n, x}\right)^{2} & \xi_{n, x} \xi_{n, y} \\
-\xi_{n, y} & \xi_{n, x} \xi_{n, y} & \left(\xi_{n, y}\right)^{2}
\end{array}\right] \\
& w_{s}\left(\mathbf{x}_{n}^{i}, \mathbf{x}_{n}^{j}\right)=e^{-\left(2\left|\xi_{n}\right| \delta\right)^{2}}
\end{aligned}
$$

The flowchart for the MLS algorithm is provided in Fig. 9. If the remainder of the current time step number $n$ divided by $n_{M L S}$ is equal to zero, an additional MLS part will be added to the algorithm provided in section 5.2 or 5.3. The density, velocity will be smoothed based on Eq. (89) and Eq.(88). As a result, the pressure and displacement will be recalculated according to the smoothed density and velocity. 


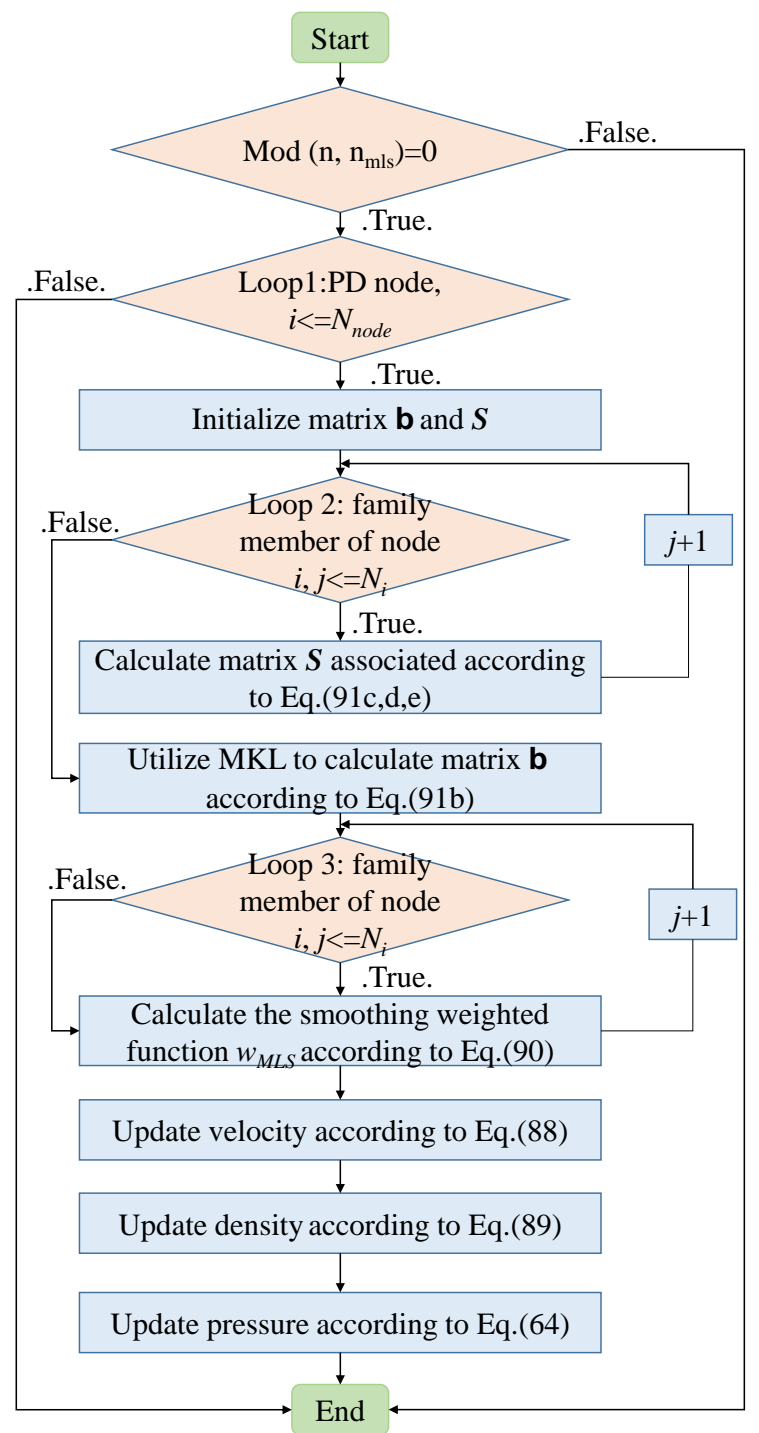

Fig. 9 Flowchart for the MLS algorithm

\section{Numerical Simulations}

In this section, numerical examples of several classical fluid flow problems are presented by using the proposed non-local PD formulations. The validation of the PD formulations is conducted by the comparisons between the PD results and those from other solutions. In all the following simulation cases, the fluid is water with density $\rho=10^{3} \mathrm{~kg} / \mathrm{m}^{3}$ and dynamic viscosity $\mu=10^{-3} \mathrm{~kg} /(\mathrm{ms})$. Furthermore, the March numbers in all cases are less than 0.3 $(M<0.3)$, leading to incompressible fluid flow. Therefore, the weakly compressible technique described in Section 4.2 is adopted for the pressure calculation.

\subsection{Couette Flow}

First, the classical Couette flow is considered which involves fluid flow between two infinite plates with a stationary initial condition. As illustrated in Fig. 10(a), the two plates (shown in orange colour) are located at $y=0$ and $y=W$ with dimensions of $L=W=10^{-3} \mathrm{~m}$. 
The two vertical edges are free and the body force is zero. At time $t=0$, the upper plate suddenly moves at a constant velocity $v_{0}=2.5 \times 10^{-5} \mathrm{~m} / \mathrm{s}$ parallel to the $x$ direction. The corresponding Reynolds number is;

$$
\operatorname{Re}=\frac{v_{0} W}{v}=2.5 \times 10^{-2}
$$

As shown in Fig. 10(b), the mesh size of the PD discretization is represented by $\Delta x$. The horizon is chosen as $\delta=3.015 \Delta x$ (Madenci et al., 2016; Madenci and Oterkus, 2014). The time step size is chosen as $10^{-6} \mathrm{~s}$ and the total simulation time is $0.6 \mathrm{~s}$. Fictitious boundary layers (represented by red nodes) with their thickness being $3 \Delta x$ (horizon) are added to implement the solid wall boundary conditions, i.e.

$$
\begin{gathered}
v_{x}(x, y<0, t)=v_{y}(x, y<0, t)=0 \\
v_{x}(x, y>W, t)=2.5 \times 10^{-5} \mathrm{~m} / \mathrm{s}, v_{y}(x, y>W, t)=0
\end{gathered}
$$

where $v_{x}$ and $v_{y}$ represent the horizontal and vertical velocities, respectively. Regarding the implementation of the other two vertical edges, if a PD node flows out of the right/left edge, it will immediately re-enter the opposite edge (Tu and $\mathrm{Li}, 2017)$. Therefore, the displacement of the material point flowing out from the edges can be modified as

$$
\begin{aligned}
& u_{x}^{\text {modified }}=u_{x}-L, \text { if } x>L \\
& u_{x}^{\text {modified }}=u_{x}+L, \text { if } x<0
\end{aligned}
$$

where $u_{x}$ represents the horizontal displacement. Finally, the total Lagrangian algorithm described in Section 5.2 is applied to this problem. 


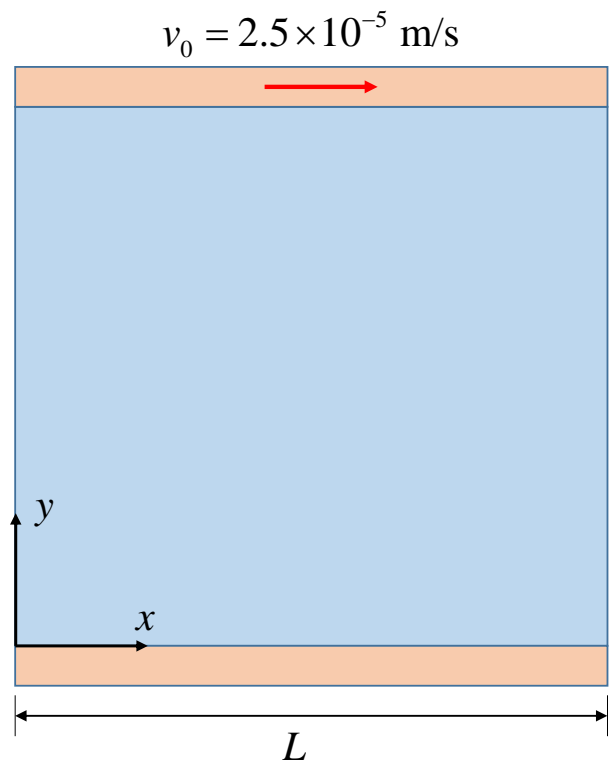

(a)

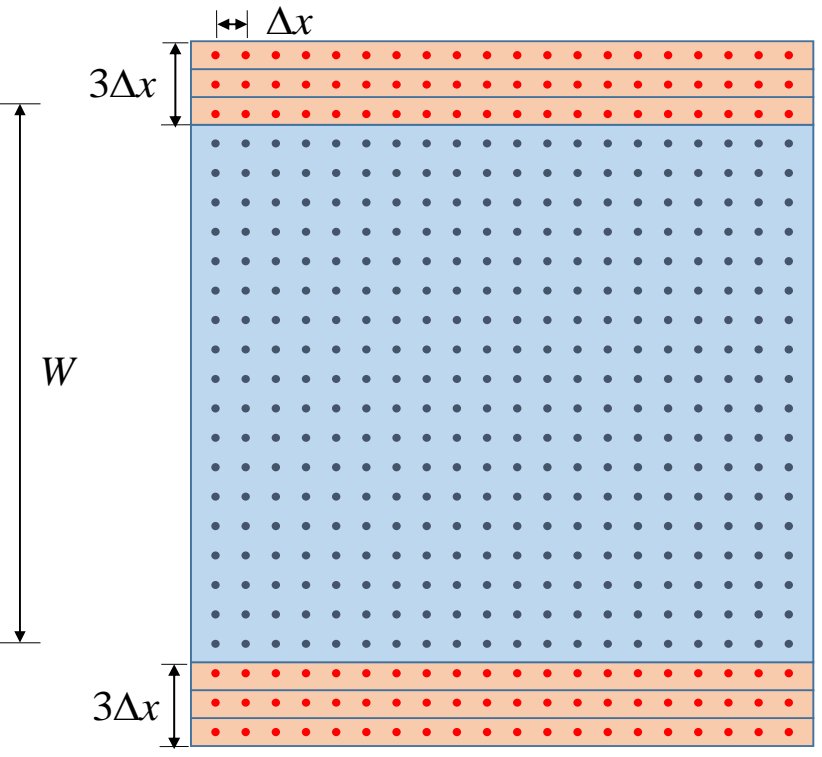

(b)

Fig. 10 Couette flow simulation illustration (a) geometry illustration and (b) PD discretization

In order to validate the proposed non-local PD model, the PD predicted results are compared with the analytical series solution provided as (Morris et al., 1997)

$$
v_{x}(y, t)=\frac{v_{0}}{W} y+\sum_{n=1}^{\infty} \frac{2 v_{0}}{n \pi}(-1)^{n} \sin \left(\frac{n \pi}{W} y\right) \exp \left(-v \frac{n^{2} \pi^{2}}{W^{2}} t\right)
$$

First, in order to estimate the sensitivity of mesh size on accuracy, a mesh convergence study is performed by using a $n \times n$ mesh where $n=50,100,200,400$. Fig. 11 shows the relative percentage error for steady state velocity at $x=L / 2$ for different mesh sizes. The relative percentage error is calculated as (Mao and Liu, 2018)

$$
\varepsilon_{r}(\%)=\sqrt{\frac{\sum\left(v_{P D}-v_{\text {Analytical }}\right)^{2}}{\sum\left(v_{\text {Analytical }}\right)^{2}}} \times 100
$$

As it can be seen from the results, as the mesh size increases the velocity value converges. Even with coarse meshes, i.e. $50 \times 50$, the relative percentage error is approximate $1.5 \%$.

Next, PD results are compared with analytical solutions provided in Eq.(95). Fig. 12 shows the comparison between the velocity profiles obtained by using the PD model and the series solution at different times by using $400 \times 400$ mesh. The steady state solution is represented at time $0.6 \mathrm{~s}$. It can be observed that the solutions from these two methods match very well, confirming the accuracy of the proposed non-local PD model. The horizontal 
velocity field for the steady state is shown in Fig. 13. Consequently, the robustness of the proposed non-local PD model in the total Lagrangian description is verified.

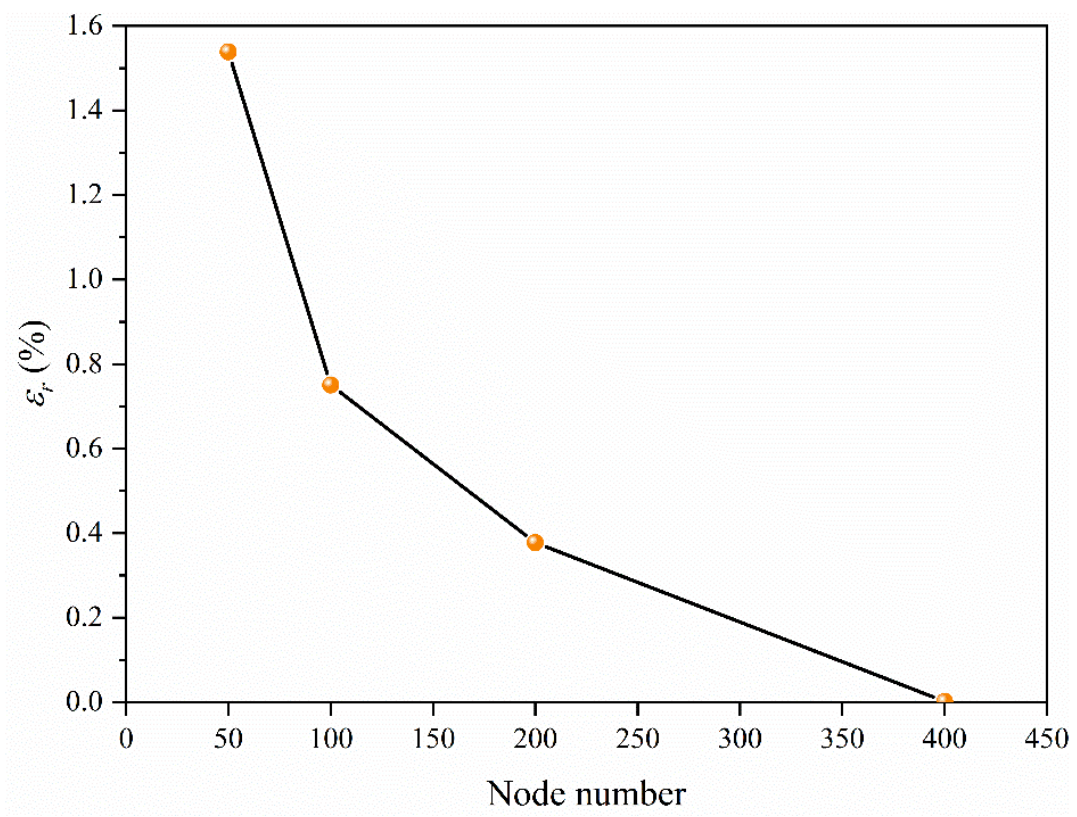

Fig. 11 Convergence study for PD solutions for Couette flow for different grid sizes at $t=0.6 \mathrm{~s}$

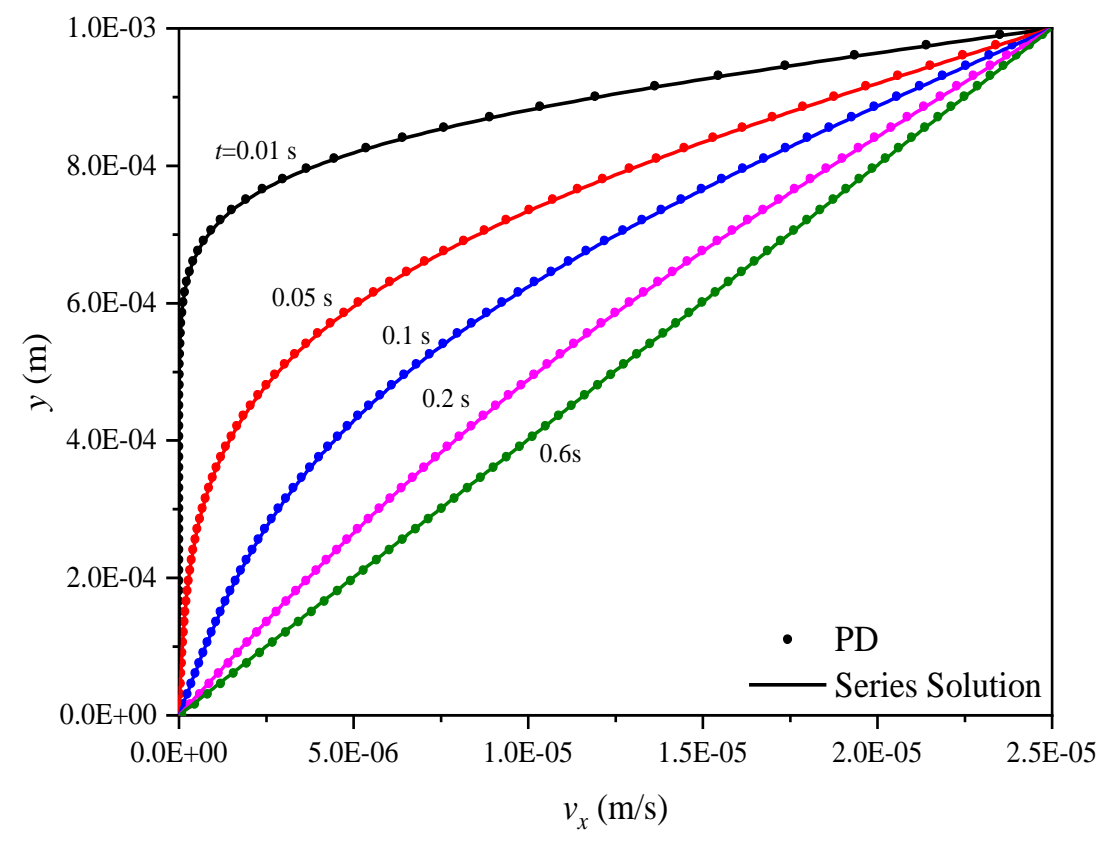

Fig. 12 Comparison of PD and series solutions for Couette flow 


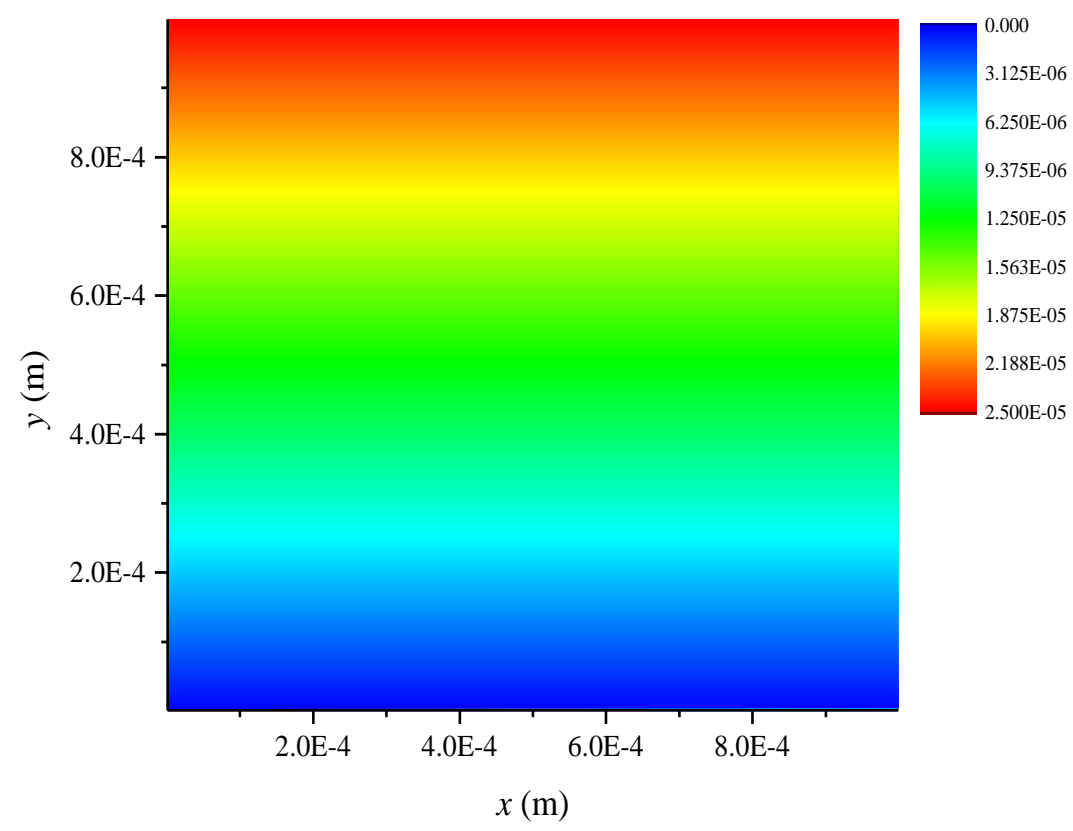

Fig. 13 Contour plot of horizontal velocity $v_{x}(\mathrm{~m} / \mathrm{s})$ predicted by PD at $t=0.6 \mathrm{~s}$

\subsection{Poiseuille Flow}

The second simulation case is Poiseuille flow between two stationary infinite plates at $y=0$ and $y=W$. The geometry is the same as the one from Couette flow, i.e. $L=W=10^{-3} \mathrm{~m}$ as shown in Fig. 10. The other two vertical edges are free. The fluid is initially at rest. Then a body force $F=2 \times 10^{-4} \mathrm{~m} / \mathrm{s}^{2}$ parallel to the $x$ direction is applied to drive the fluid to flow gradually, leading to a steady state flow distribution finally.

In the numerical implementation, the same PD discretization model is adopted by using $400 \times 400$ mesh in the flow region with the horizon chosen as $\delta=3.015 \Delta x$. The constant time step size is $10^{-6} \mathrm{~s}$ and the total simulation time is $0.6 \mathrm{~s}$. The solid wall boundary condition is implemented by using the fictitious layer as illustrated in Fig. 10 (b) is applied as

$$
\begin{gathered}
v_{x}(x, y<0, t)=v_{y}(x, y<0, t)=0 \\
v_{x}(x, y>W, t)=v_{y}(x, y>W, t)=0
\end{gathered}
$$

Besides, the vertical boundary implementation approach provided in Eq. (97) is also utilized. The total Lagrangian method described in Section 5.2 is adopted.

In order to validate the proposed PD model, the simulation results are compared with the time-dependent series solution provided as (Morris et al., 1997)

$$
v_{x}(y, t)=\frac{F}{2 v} y(y-W)+\sum_{n=0}^{\infty} \frac{4 F W^{2}}{v \pi^{3}(2 n+1)^{3}} \sin \left(\frac{\pi y}{W}(2 n+1)\right) \exp \left(-v \frac{(2 n+1)^{2} \pi^{2}}{W^{2}} t\right)
$$


The horizontal velocity profiles obtained by the PD model are compared with the analytical solutions provided in Eq. (98), as shown in Fig. 14. Good agreements are also obtained in this case, validating the proposed non-local PD model. The horizontal velocity field is provided in Fig. 15 at time $0.6 \mathrm{~s}$. As it can be seen from the results, the maximum velocity is obtained as $v_{0}=2.5 \times 10^{-5} \mathrm{~m} / \mathrm{s}$ which corresponds to the Reynold number as $\operatorname{Re}=2.5 \times 10^{-2}$.

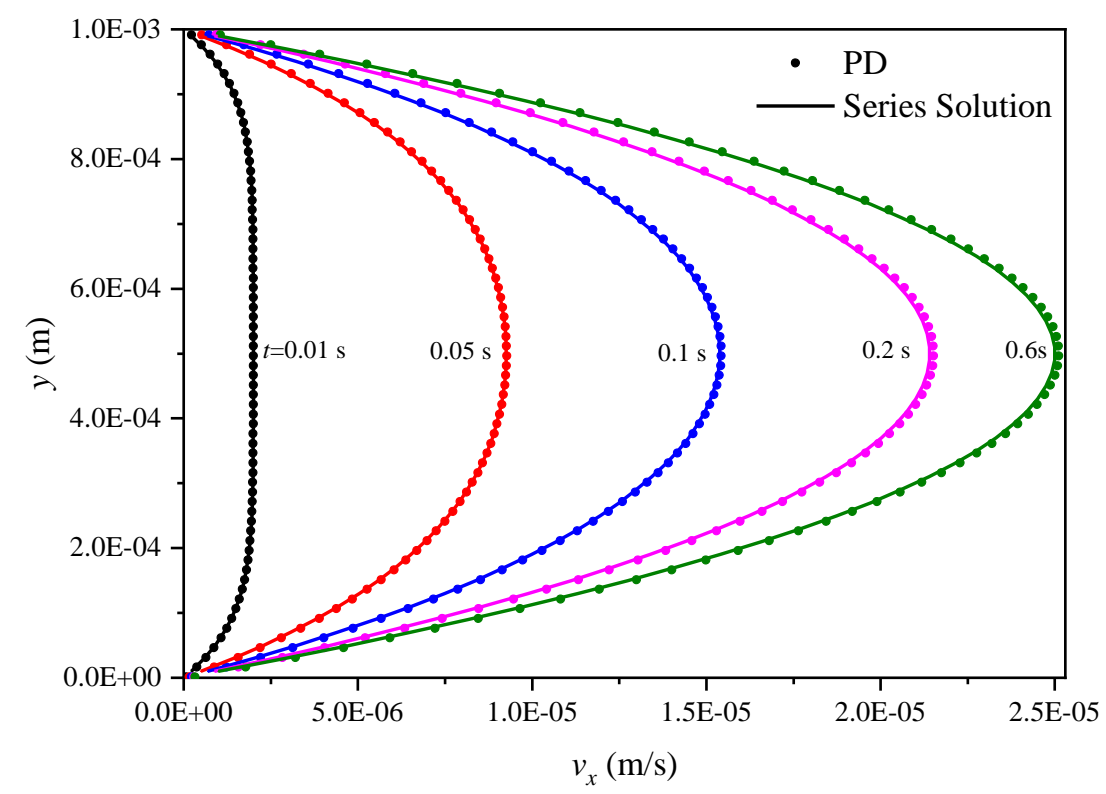

Fig. 14 Comparison of PD and series solutions for Poiseuille flow

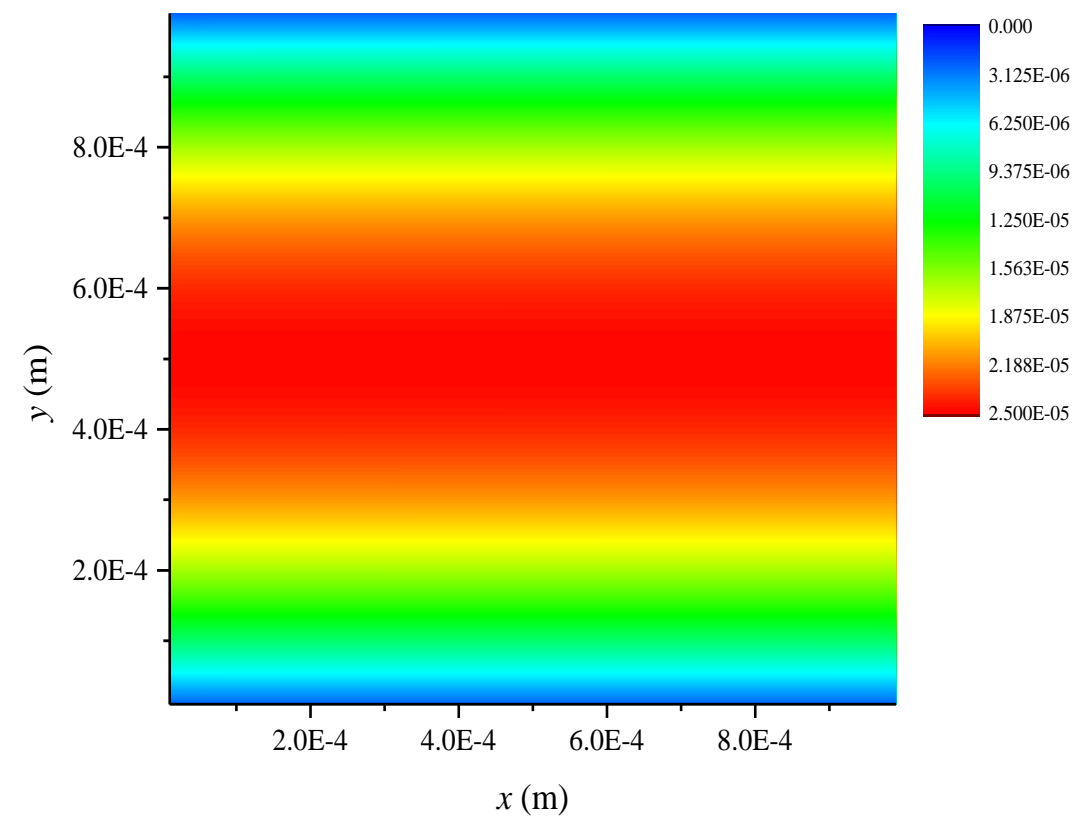

Fig. 15 Contour plot of horizontal velocity $v_{x}(\mathrm{~m} / \mathrm{s})$ predicted by PD at $t=0.6 \mathrm{~s}$

\subsection{Taylor-Green Vortex}


As a third simulation case, the two-dimensional Taylor-Green vortex at $\mathrm{Re}=1$ is simulated by the proposed PD model in a total Lagrangian description. The geometry of the fluid field is set as $[0,1] \times[0,1]$. The analytical solution of the velocity field is given as

$$
\begin{gathered}
v_{1}(x, y, t)=-v_{0} e^{b t} \cos (2 \pi x) \sin (2 \pi y) \\
v_{2}(x, y, t)=v_{0} e^{b t} \sin (2 \pi x) \cos (2 \pi y)
\end{gathered}
$$

where the decay rate is $b=-8 \pi^{2} / \operatorname{Re}$ (Adami et al., 2013) and $v_{0}$ is the maximum initial velocity magnitude.

As shown in Fig. 16, four fictitious layers with their thicknesses being horizon are added outside the fluid field. The fictitious particles (shown in grey colour) are assorted into eight regions (numbered by yellow colour). Their physical parameters such as velocity etc. are forced to be equal to the fluid particles (shown in orange colour) in the same corresponding numbered region (shown in red colour). Therefore, the periodic boundary conditions in both $x$ and $y$ directions are implemented. As to the initial condition, the analytical velocity distribution at $t=0$ is used with $v_{0}=0.04$ as

$$
\begin{gathered}
v_{1}(x, y, 0)=-0.04 \cos (2 \pi x) \sin (2 \pi y) \\
v_{2}(x, y, 0)=0.04 \sin (2 \pi x) \cos (2 \pi y)
\end{gathered}
$$

The time step size is chosen as $\mathrm{d} t=1 \times 10^{-5}$ and the simulation time is $t=0.05$. Three different mesh sizes are chosen as $\Delta x=1 / n$ with $n=40,50,100$ to conduct the convergence study.

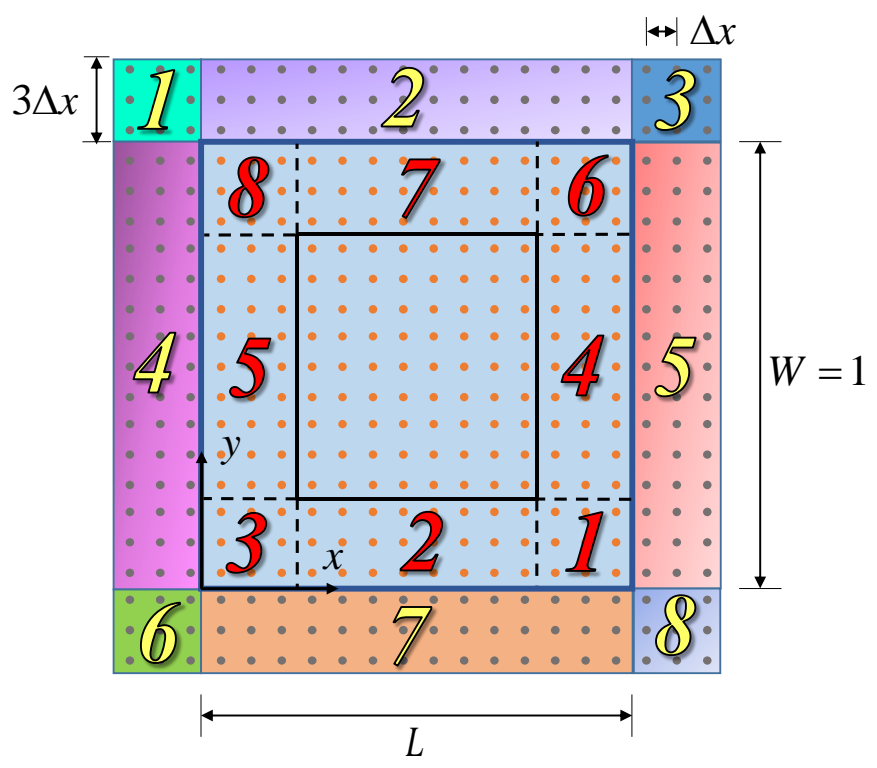

Fig. 16 Coordinate definition and boundary implementation for the Taylor Green vortex PD

A comparison of the decay of the maximum velocity magnitude is provided in Fig. 17. The exact solution and the remeshed smoothed particle hydrodynamics (rSPH) solution 
(Chaniotis et al., 2002) are provided for comparison. It can be inferred that the PD predicted results agree well with the exact and rSPH solution. For the error analysis of the PD simulations, the relative error norm $L_{\infty}$ is calculated as (Adami et al., 2013; Chaniotis et al., 2002)

$$
L_{\infty}=\max _{t=0} T_{\max }\left(\left|\frac{\max \left(\left|\mathbf{v}_{i}(t)\right|\right)-v_{0} e^{b t}}{v_{0} e^{b t}}\right|\right)
$$

with $T_{\max }$ being the time where $\left(v_{0} e^{b t}\right)=v_{0} / 50$ (Chaniotis et al., 2002). The relative errors obtained by $40 \times 40,50 \times 50$, and $100 \times 100$ particles are provided in Fig. 18. The PD predicted velocity magnitude distributions obtained by PD and exact solutions at $t=0.05$ with $100 \times 100$ particles are shown in Fig. 19 .

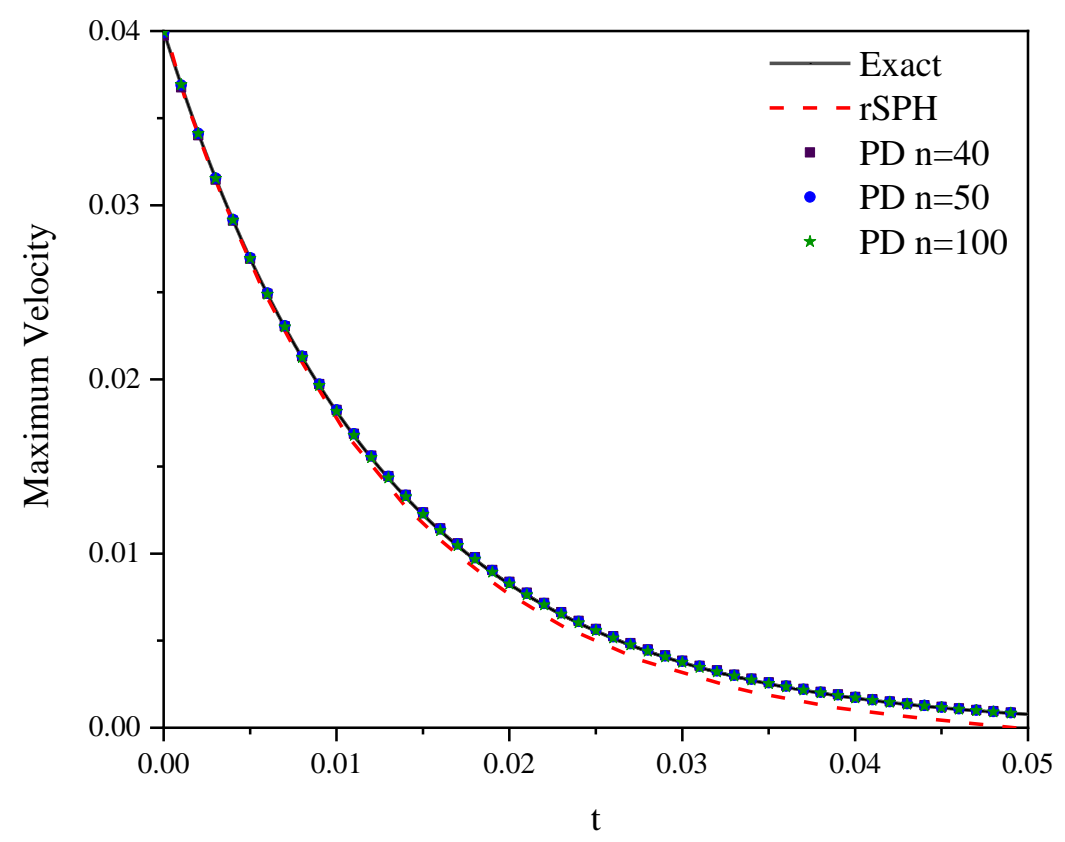

Fig. 17 Comparison of the maximum velocity between exaction solutions, rSPH solutions (Chaniotis et al., 2002) and PD solutions. 


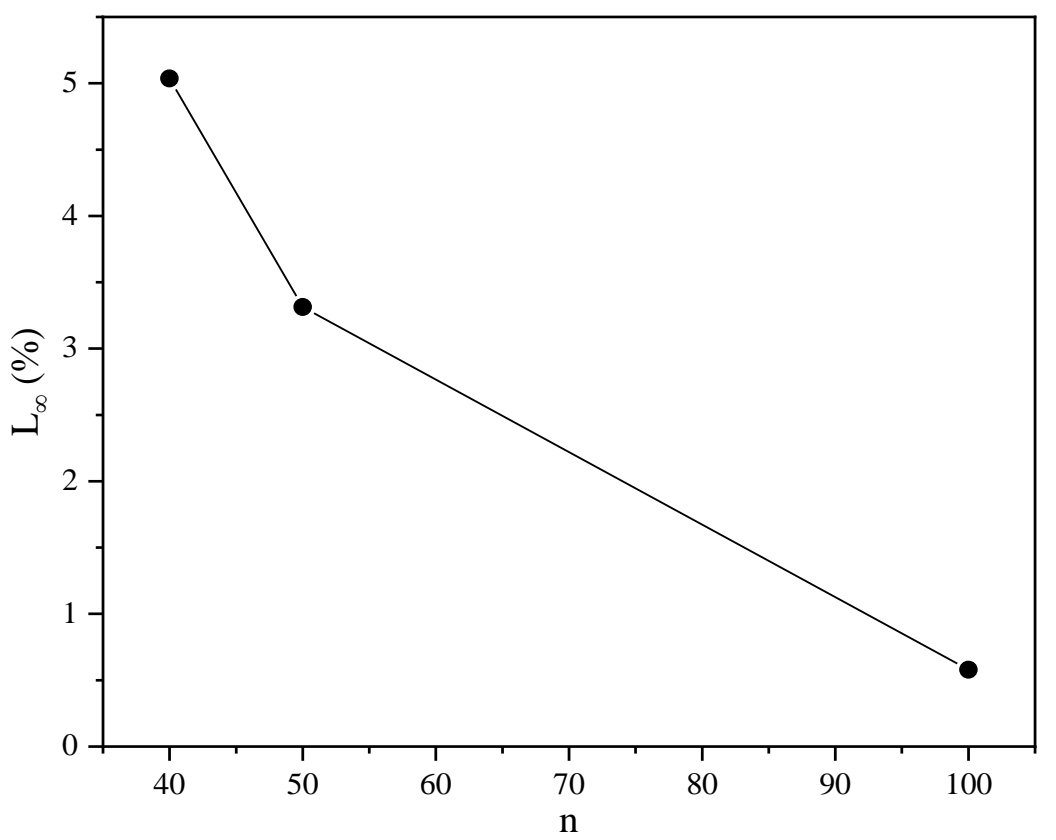

Fig. 18 Relative error of maximum velocity for different mesh sizes as $n=40,50,100$

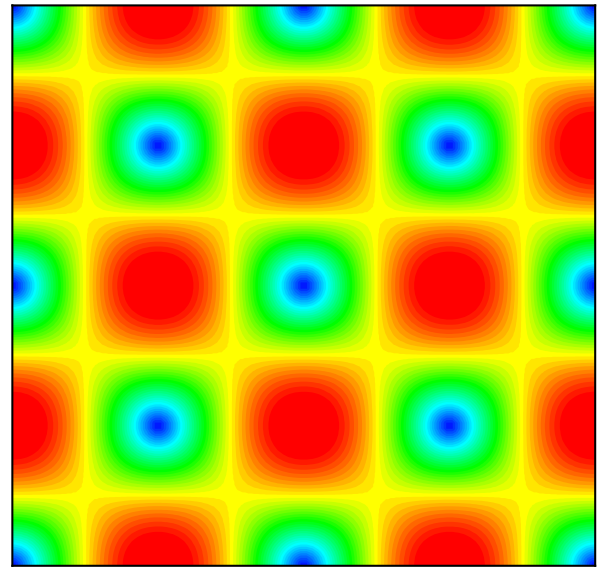

(a)

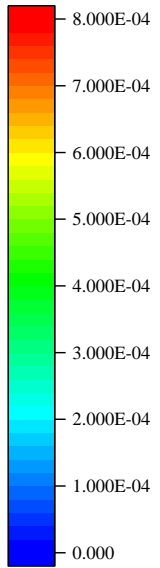

.000

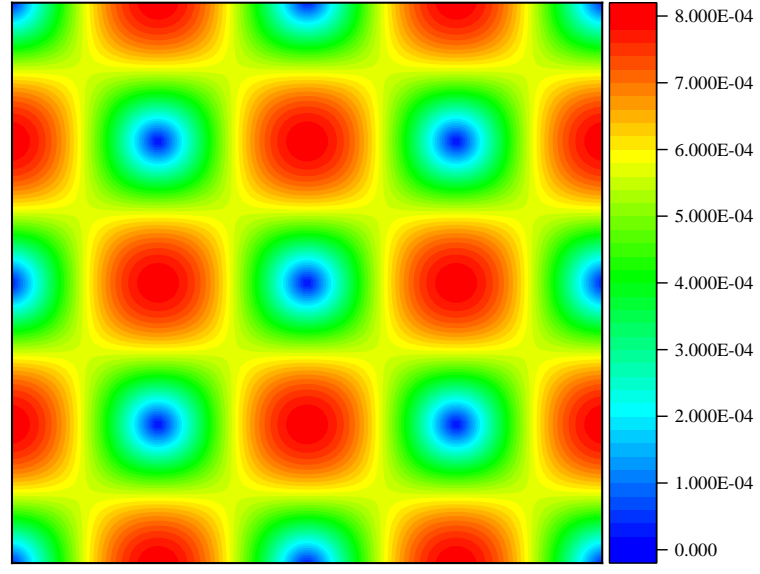

(b)

Fig. 19 The velocity magnitude distribution at $t=0.05$ with $100 \times 100$ particles for (a) PD solution and (b) exact solution.

\subsection{Shear-driven Cavity Problem}

The fourth simulation is regarding the shear-driven cavity problem. The fluid is initially at rest within a closed square cavity. The fluid flow is generated by moving the top side of the square cavity at a constant velocity, $v_{0}=10^{-3} \mathrm{~m} / \mathrm{s}$ parallel to the $x$ direction. As illustrated in Fig. 20, the geometry dimension is set as $L=W=10^{-3} \mathrm{~m}$. The PD mesh size is chosen as $\Delta x=L / n$ with $\mathrm{n}$ represents the particle number in one direction as $n=40,100$ for PD 
simulations. The horizon size $\delta=3.015 \Delta x$ is adopted. The time step size is chosen as $10^{-4} \mathrm{~s}$ and the total simulation time is $0.3 \mathrm{~s}$. The solid boundary conditions are implemented as described in Section 5.4 (Eq.(82), Eq.(84), Eq.(85)) by using fictitious layer, as shown in Fig. 20. During the simulation, the updated Lagrangian method described in Section 5.3 is adopted.

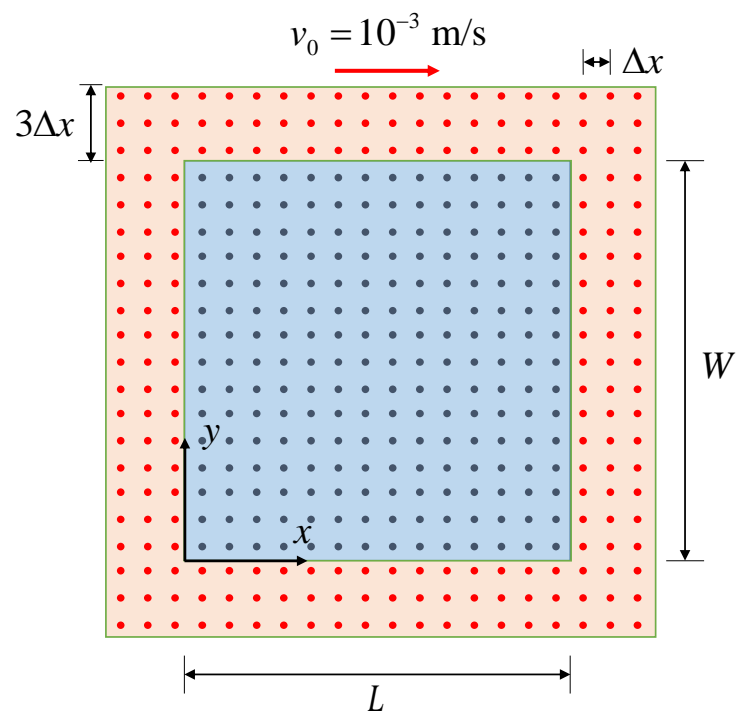

Fig. 20 Illustration of PD discretization for the shear-driven cavity problem

In order to validate the proposed PD model, the velocity profiles in steady state condition, at $t=0.3 \mathrm{~s}$ are compared with the previous literature. The reference data in (Liu, 2011) obtained by the finite difference method (FDM) and smoothed particle hydrodynamics (SPH) and in (Mao and Liu, 2018) obtained by a Lagrangian gradient smoothing method (L-GSM) is provided in Fig. 21. In addition, the PD solution by different mesh sizes as $40 \times 40$ and $100 \times 100$ are also provided. It can be observed that the PD results are close to the ones obtained by these two methods, proving the accuracy of the proposed PD model for the shear driven cavity problem. 


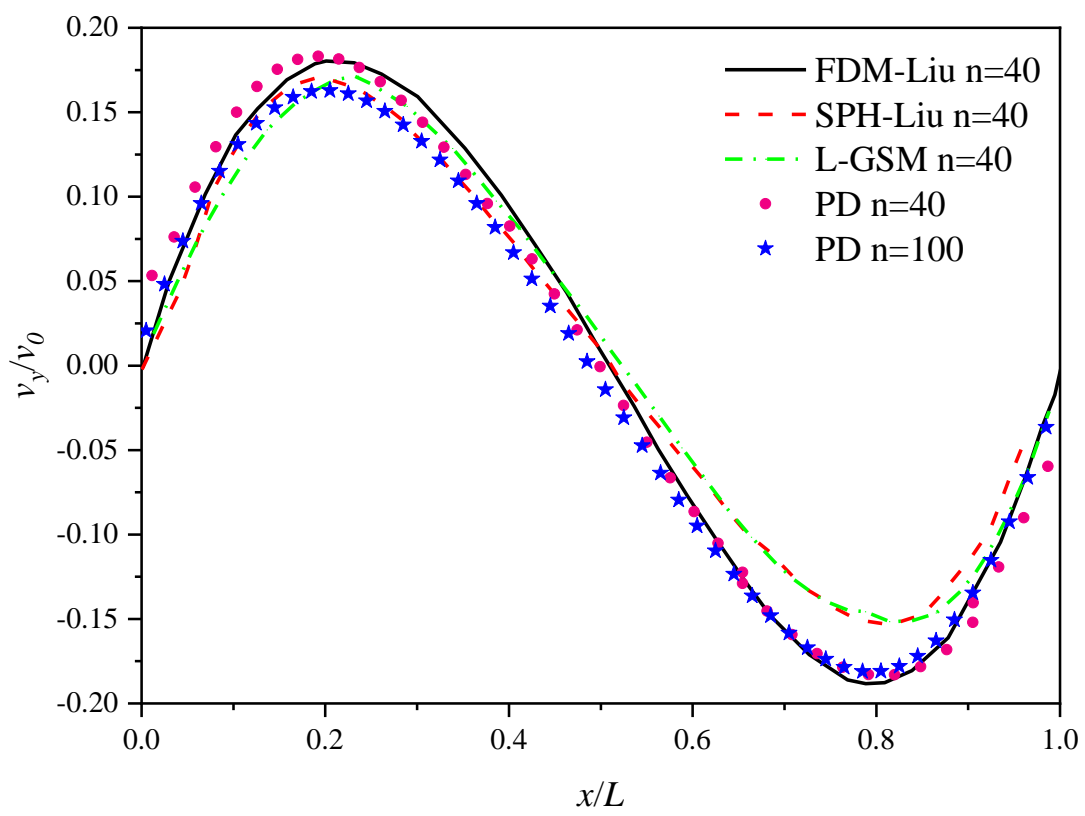

(a)

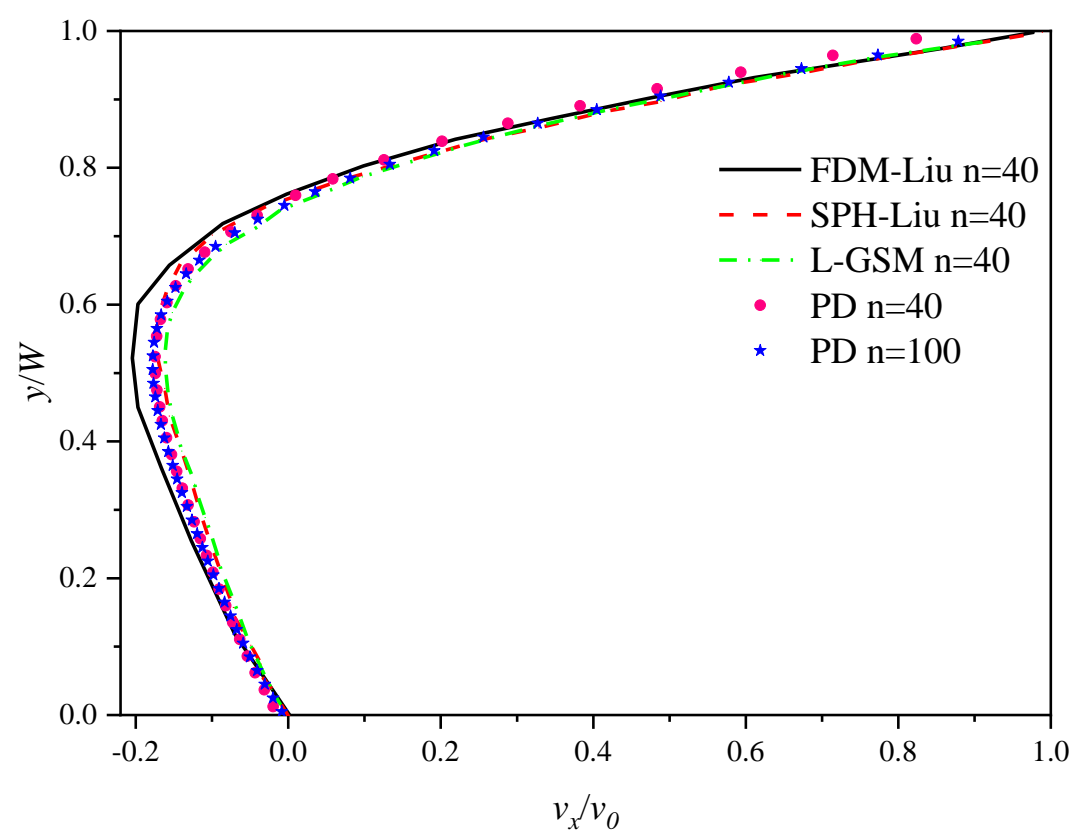

(b)

Fig. 21 Comparison of non-dimensional steady state PD velocity profiles with FDM and SPH data (Liu, 2011), L-GSM data (Mao and Liu, 2018) (a) vertical velocity at $y=W / 2$ (b) horizontal velocity at $x=L / 2$

The horizontal velocity distributions for each material point at time $t=0.05 \mathrm{~s}$ and $t=0.3 \mathrm{~s}$ are presented in Fig. 22. The vertical velocity vector field at time $t=0.05 \mathrm{~s}$ and $t=0.3 \mathrm{~s}$ are also provided in Fig. 23. It is observed that the fluid flow is in a recirculation pattern within the closed square finally reaches its steady state form. 


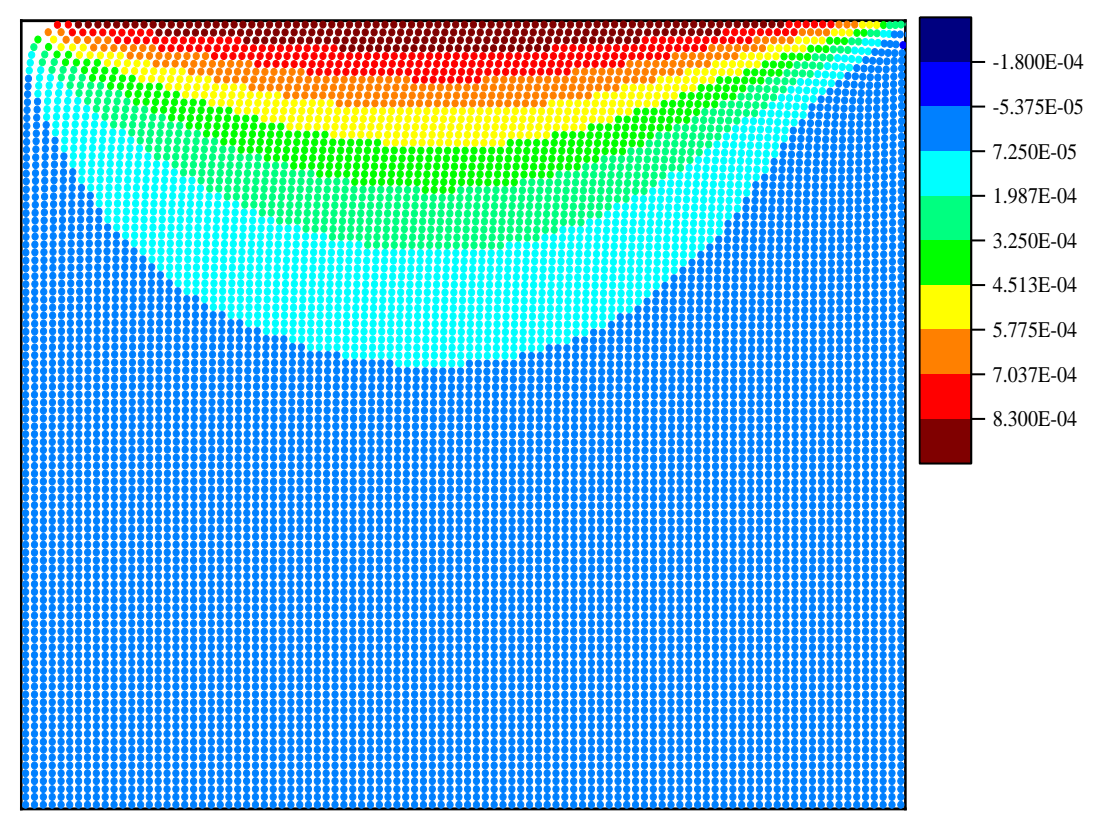

(a)

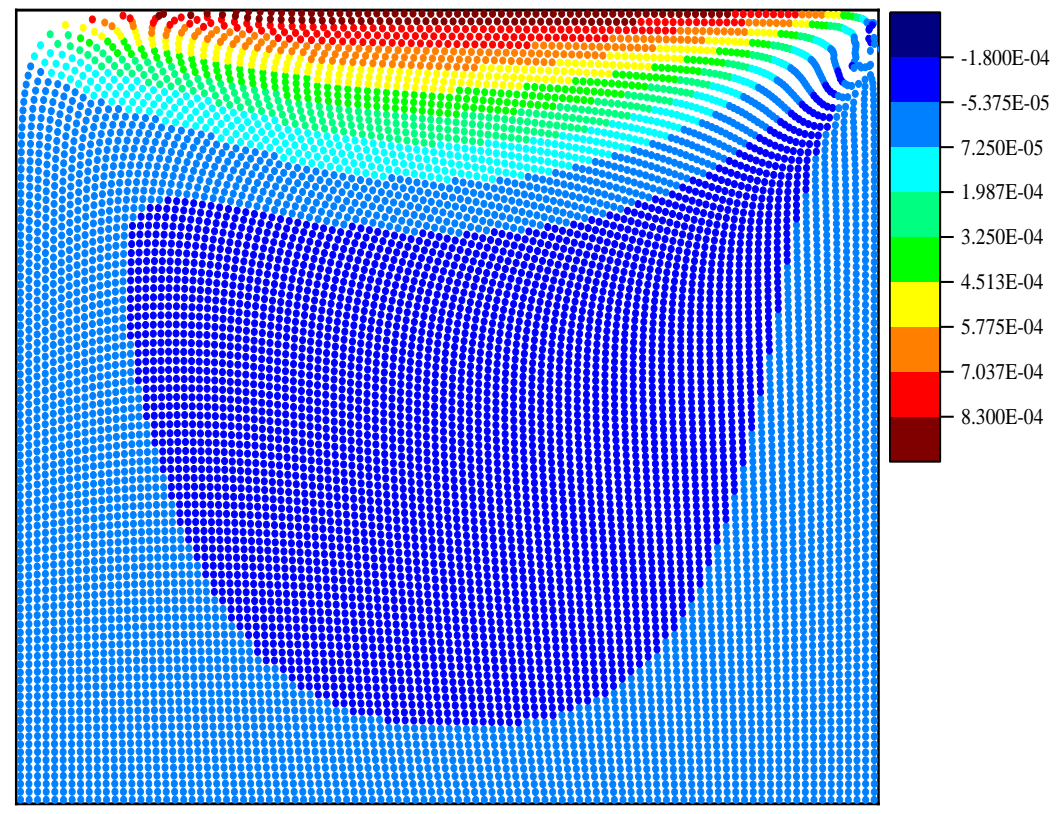

(b)

Fig. 22 Horizontal velocity $v_{x}(\mathrm{~m} / \mathrm{s})$ distribution and the particle positions predicted by PD for $100 \times 100$ mesh size at (a) $t=0.05 \mathrm{~s}$ and (b) at $t=0.3 \mathrm{~s}$ 


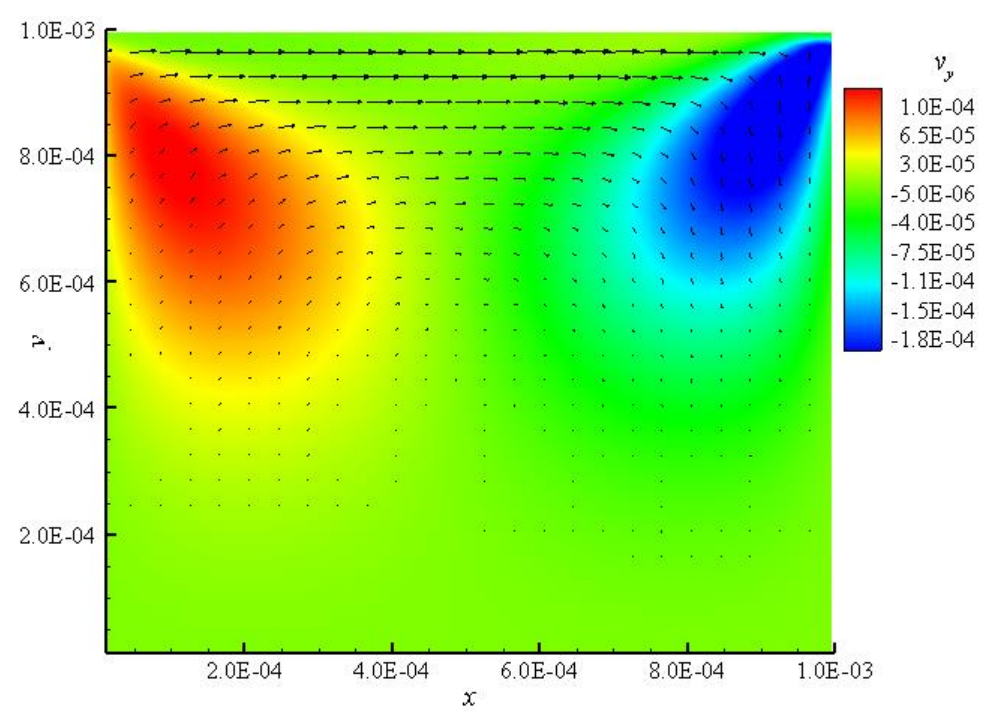

(a)

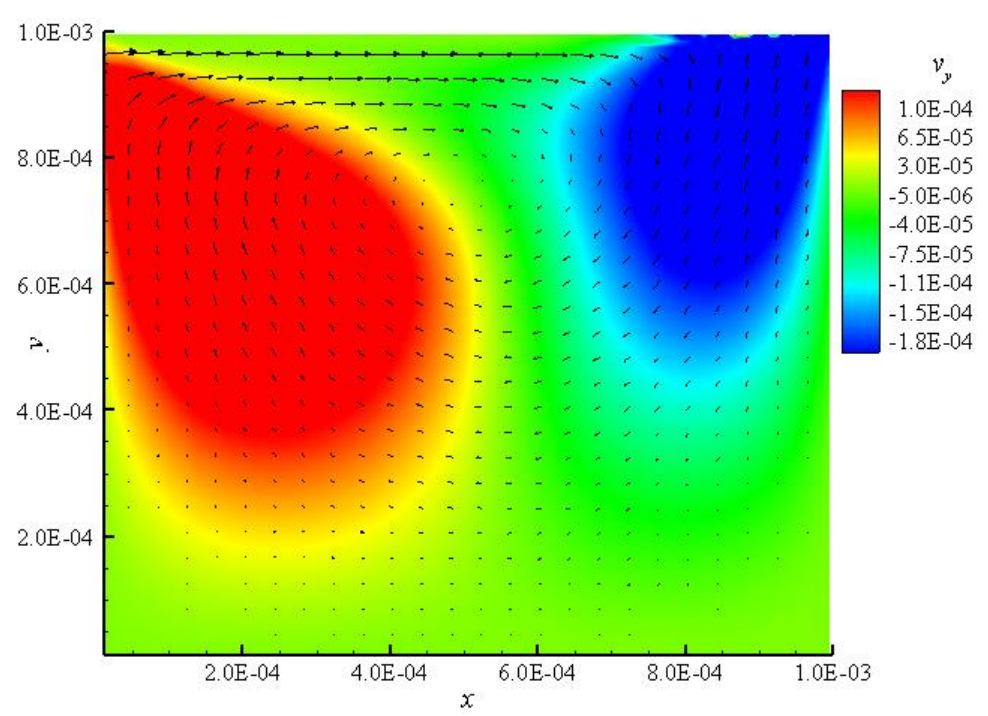

(b)

Fig. 23 Velocity vector field coloured by their vertical component magnitude predicted by PD

$$
\text { for } 100 \times 100 \text { mesh size at (a) } t=0.05 \mathrm{~s} \text { (b) } t=0.3 \mathrm{~s}
$$

\subsection{Dam Collapse Problem}

To demonstrate the capability of the proposed PD formulations for predicting free surface flows, the classical dam collapse problem which has been extensively investigated (Colagrossi and Landrini, 2003; Wang and Zhang, 2018) is considered in this section. The dam break experiment conducted by S. Koshizuka and Y. Oka (Koshizuka and Oka, 1996) is used for comparison. A two-dimensional flow generated after the breaking of a dam is simulated as 
shown in Fig. 24(a). The geometry dimensions of the water column are $L=0.146 \mathrm{~m}$ for width and $2 L$ for height. The tank is open at the top with its width and height being $4 L$ and $L$ respectively. The flow is driven by the gravity and it is constraint by an open rectangle square. The gravitational acceleration is set as $g=9.8 \mathrm{~m} / \mathrm{s}^{2}$. Regarding the PD implementation shown in Fig. 24(b), the mesh size is chosen as $\Delta x=4.867 \times 10^{-3} \mathrm{~m}(30 \times 60$ fluid particles $)$ and the horizon is chosen as $\delta=4.015 \Delta x$. The time step size is $\mathrm{d} t=2.5 \times 10^{-5} \mathrm{~s}$ with the total simulation time $0.4 \mathrm{~s}$. As shown in Fig. 24(b), fictitious layers (thickness being horizon) are added to implement the wall boundary conditions (shown in red colour). The acceleration, velocity, and displacements of the fictitious wall particles are set to be zero according to Eq. (82). The pressure and density of these fictitious wall particles are calculated according to Eq. (84) and Eq.(85). In this problem, $B$ parameter in Eq. (43) is calculated by approximating the maximum magnitude of fluid flow velocity as $\left|\mathbf{v}_{f}\right|=\sqrt{2 g(2 L)}=2.39 \mathrm{~m} / \mathrm{s}$ (Monaghan, 1994), leading to $B=8.16 \times 10^{4} \mathrm{~Pa}$. Also, zero pressure condition is applied for the free surface fictitious layers (shown in green colour) according to Eq.(86). The acceleration, velocity, displacement, and density of these free surface particles are calculated as real fluid particles. Besides, the updated Lagrangian description in section 5.3 is adopted. The damping time in Eq.(87) to avoid the initial sudden movement is chosen as $t_{\text {damp }}=100 \mathrm{~d} t$. The MLS method described in Section 5.4.2 is applied with $n_{M L S}=60$ for $t \leq 0.3 \mathrm{~s}$ and $n_{M L S}=15$ for $t>0.3 \mathrm{~s}$ (when the water splashes the right vertical wall). 


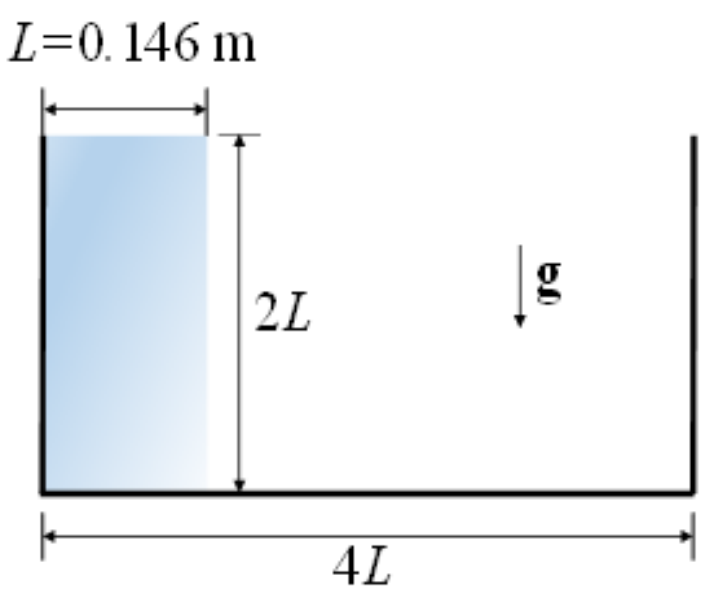

(a)

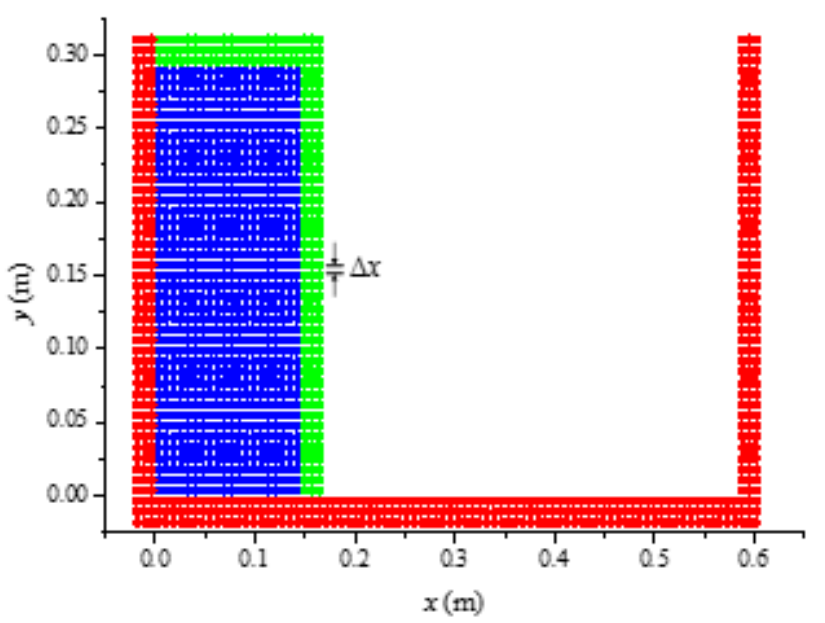

(b)

Fig. 24 Sketch of the dam collapse problem (a) geometry model and (b) PD discretization model

Snapshots of vertical velocity profiles and the particle positions at different times are provided in Fig. 25-28 for both PD and experimental results. As shown in Fig. 29, the $x$ position of the dame toe obtained by the PD simulation agrees well with the experiment results (Koshizuka, 1995; Martin et al., 1952). It can be inferred that the proposed PD model is capable of predicting the classical dam collapse problem. It can be inferred from the numerical simulation results that there is void in the lower right corner of the fluid. The reason may be due to boundary pressure treatment for free surfaces. The pressures of the particles for free surfaces are directly set to be zero, while the pressure of the other fluid particles are calculated according to the equation of state. As a result, it may produce discontinuities in the pressure profiles and lead to the voids in the lower right corner of the water column. In addition, the utilization of the MLS method may also bring some error and discontinuities on the fluid motion. The boundary treatments can be further improved by using constrained conditions for the fictituous layers (Oterkus, 2015; Oterkus et al., 2014) or by incorporating the boundary conditions into the governing equations (Madenci et al., 2016). 


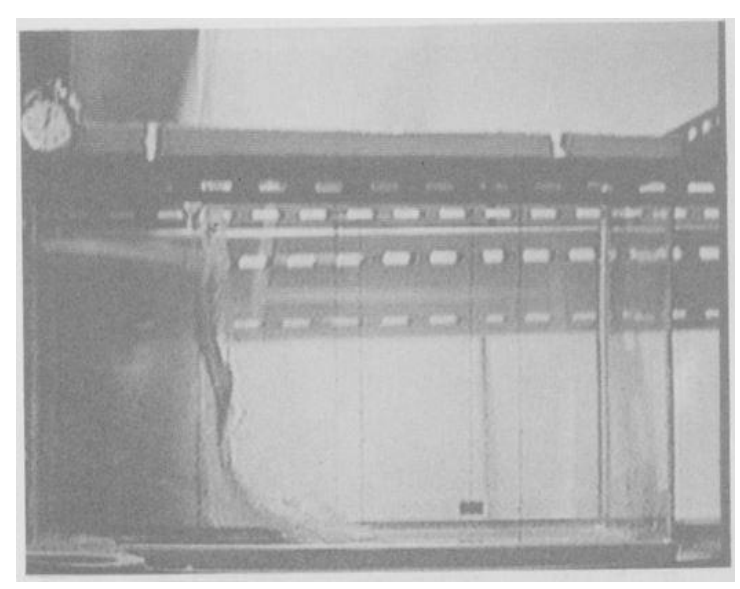

(a)

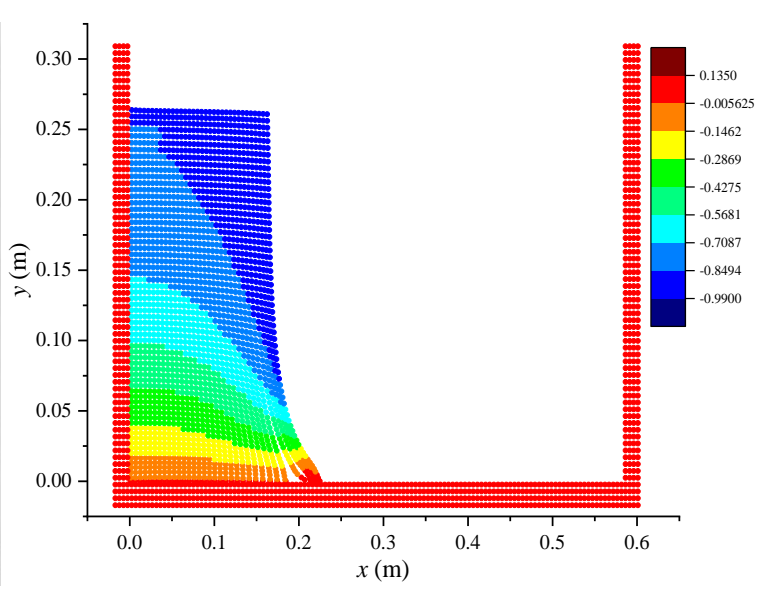

(b)

Fig. 25 Comparison between (a) experimental result (Koshizuka and Oka, 1996) and (b) PD predictions coloured by vertical velocity $(\mathrm{m} / \mathrm{s})$ at $t=0.1 \mathrm{~s}$

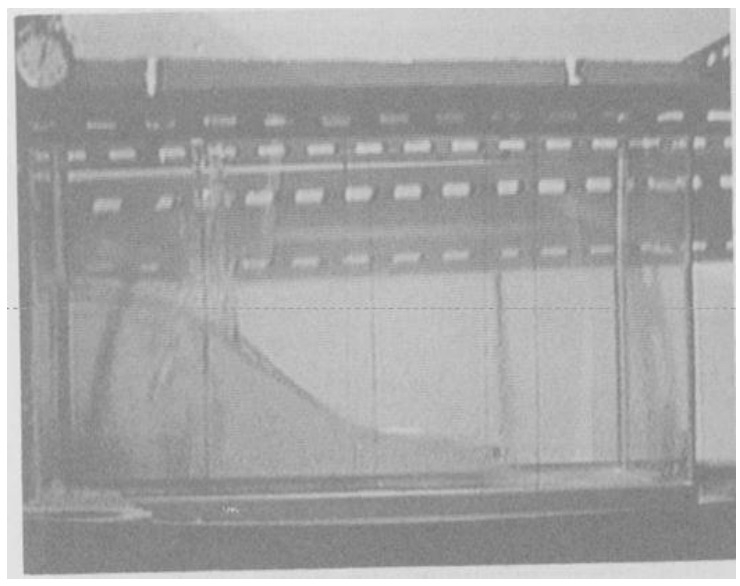

(a)

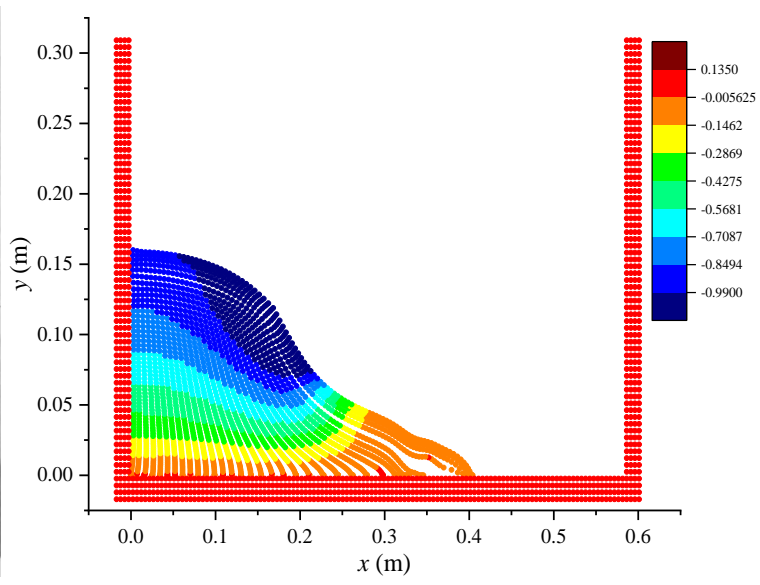

(b)

Fig. 26 Comparison between (a) experimental result (Koshizuka and Oka, 1996) and (b) PD predictions coloured by vertical velocity $(\mathrm{m} / \mathrm{s})$ at $t=0.2 \mathrm{~s}$
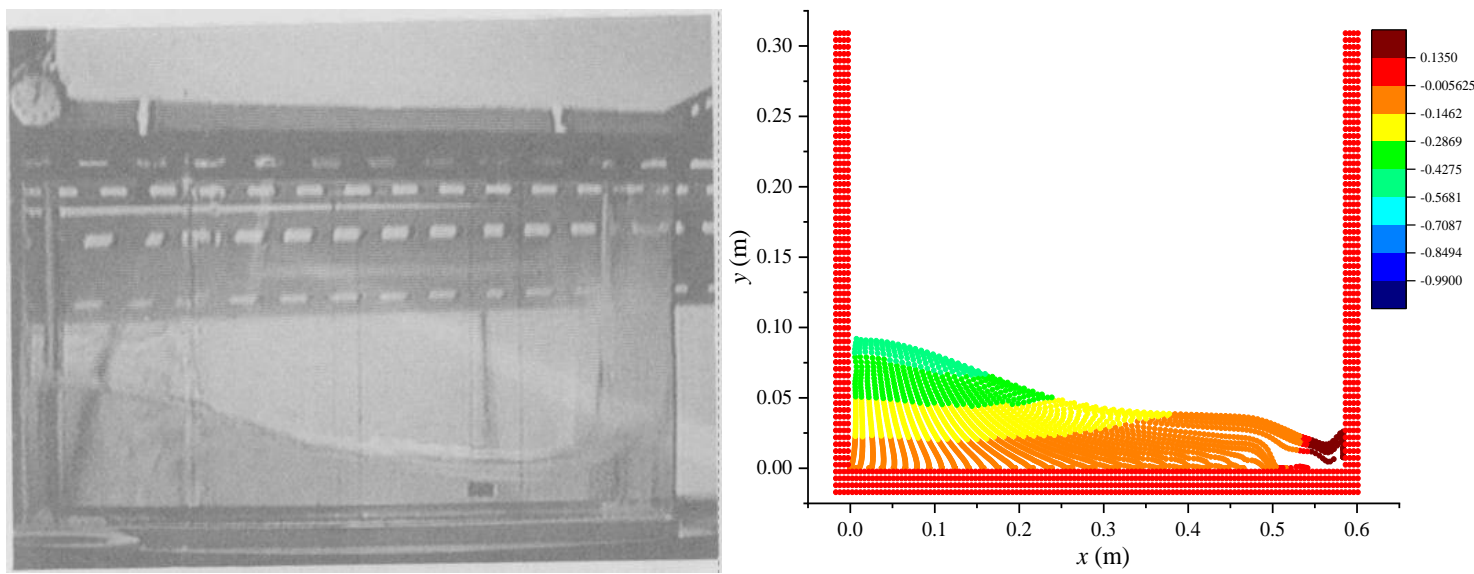

Fig. 27 Comparison between (a) experimental result (Koshizuka and Oka, 1996) and (b) PD predictions coloured by vertical velocity $(\mathrm{m} / \mathrm{s})$ at $t=0.3 \mathrm{~s}$ 

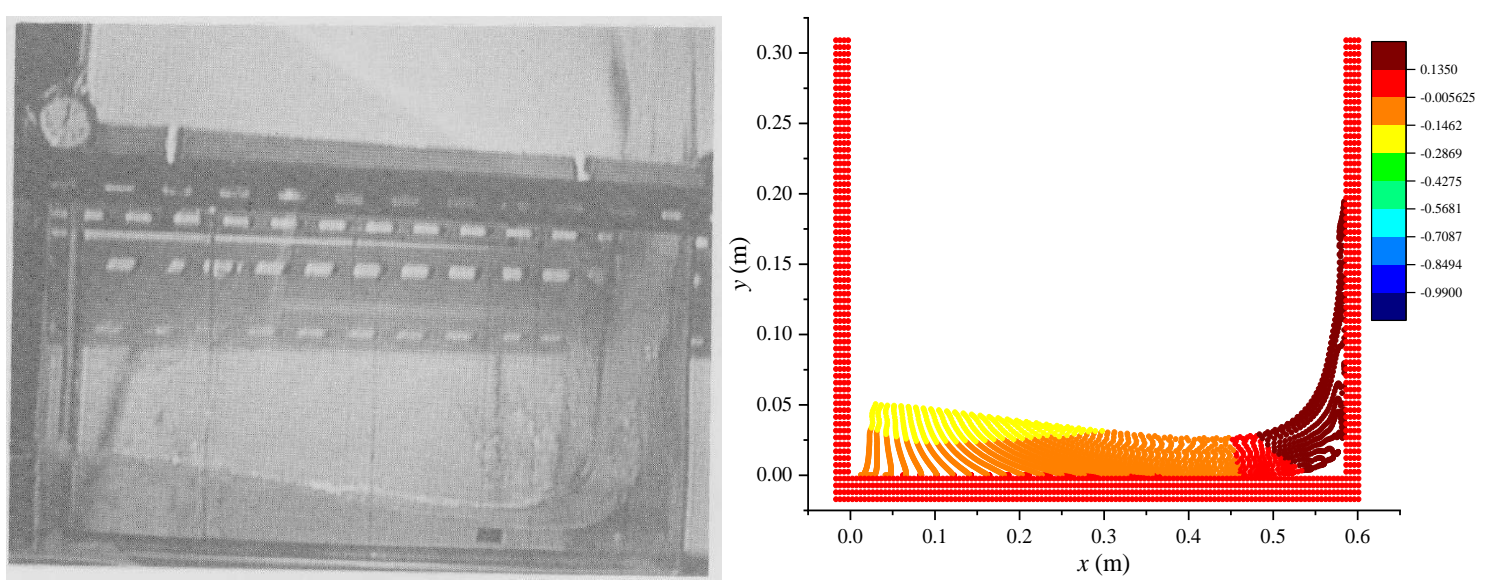

Fig. 28 Comparison between (a) experimental result (Koshizuka and Oka, 1996) and (b) PD predictions coloured by vertical velocity $(\mathrm{m} / \mathrm{s})$ at $t=0.4 \mathrm{~s}$

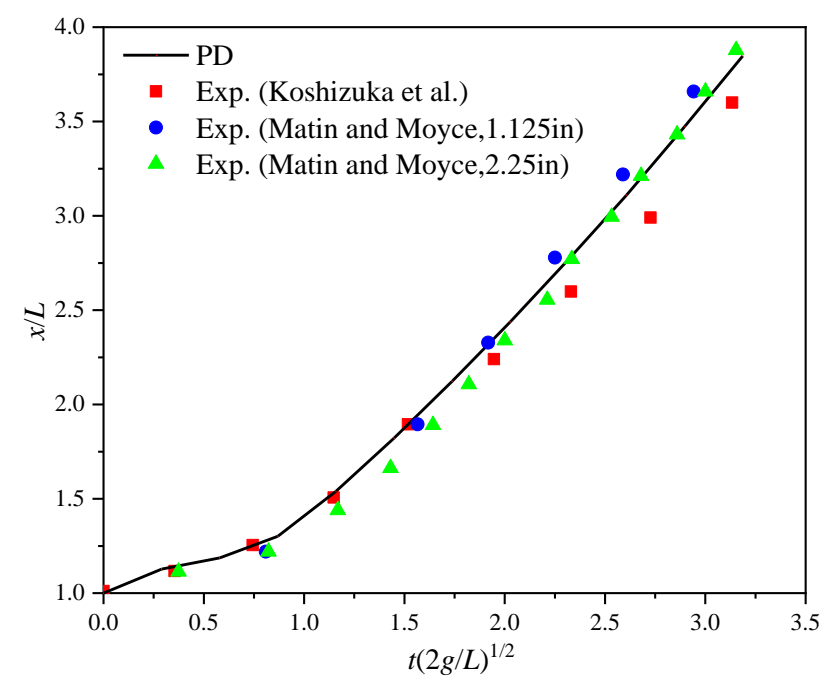

Fig. 29 Comparison of the $\mathrm{X}$ position of the dam toe for PD and experiment results

(Koshizuka, 1995; Martin et al., 1952)

\section{Conclusion}

In this paper, the fluid flow governing equations, i.e. Navier-Stokes equations, are reformulated into an integral form by using the peridynamic differential operator. Subsequently, the numerical algorithm both in total and updated Lagrangian descriptions are provided. Several classical fluid flow problems, i.e. Couette flow, Poisuelle flow, Talyor-Green Vortex, shear-driven cavity problem, are simulated by the proposed PD model. Furthermore, the developed PD model is applied to solve problems of free-surface flow as presented in the dam collapse problem. The PD predicted results agree well with those obtained from other solutions, validating the capability of the proposed model for solving hydrodynamics including free surface flows problems. The present model can be further developed for other fluid flow problems such as multi-phase or multi-component fluid flow problems. Furthermore, since there are many existing PD models for solids, the fluid-structure interaction can be 
straightforwardly implemented by using the non-local models both for the fluid and the solid. The interaction can be inherently implemented because of the models' non-local behaviour.

\section{Acknowledgment}

The authors gratefully acknowledge financial support from the China Scholarship Council (CSC No. 201506230126) and University of Strathclyde.

\section{Appendix A}

\section{PD Differential Operator for 2D Problems}

In this study, two dimensional (2D) fluid flow is simulated. Therefore, peridynamic differential operator for two-dimensional space up to second order derivative is provided.

\section{PD differential operator up to second derivative}

Similar to Eq. (2a), Taylor series expansion up to second order derivatives for twodimensional space is expressed in an explicit form as

$$
\begin{aligned}
f(\mathbf{x}+\xi)-f(\mathbf{x}) & =\xi_{1} \frac{\partial f(\mathbf{x})}{\partial x_{1}}+\xi_{2} \frac{\partial f(\mathbf{x})}{\partial x_{2}}+\frac{1}{2} \xi_{1}^{2} \frac{\partial^{2} f(\mathbf{x})}{\partial x_{1}^{2}} \\
& +\frac{1}{2} \xi_{2}^{2} \frac{\partial^{2} f(\mathbf{x})}{\partial x_{2}^{2}}+\xi_{1} \xi_{2} \frac{\partial^{2} f(\mathbf{x})}{\partial x_{1} \partial x_{2}}+R_{\mathbf{x}, 2}(\xi)
\end{aligned}
$$

where $R_{\mathbf{x}, 2}(\xi)$ is the remainder for Taylor series as

$$
R_{\mathbf{x}, 2}(\xi)=\sum_{\left|n_{1}+n_{2}\right|=3} \frac{1}{n_{1} ! n_{2} !} \frac{\partial^{3} f(\mathbf{x}+\alpha \xi)}{\partial x_{1}^{n_{1}} \partial x_{2}^{n_{2}}} \xi_{1}^{n_{1}} \xi_{2}^{n_{2}}, \text { for some } \alpha \in(0,1)
$$

Note that $R_{\mathbf{x}, 2}(\xi)=O\left(|\xi|^{3}\right)$ and it is assumed to be negligible. The non-local form with the help of PD differential operator will be derived for following differentials.

$$
\frac{\partial f(\mathbf{x})}{\partial x_{1}}, \frac{\partial f(\mathbf{x})}{\partial x_{2}}, \frac{\partial^{2} f(\mathbf{x})}{\partial x_{1}^{2}}, \frac{\partial^{2} f(\mathbf{x})}{\partial x_{2}^{2}}, \frac{\partial^{2} f(\mathbf{x})}{\partial x_{1} \partial x_{2}}
$$

Since the derivatives are up to the second derivative, PD differential operator $g^{p_{1} p_{2}}(\xi)$ will be used to represent the PD nonlocal expressions up to the second derivative.

Multiplying $g^{p_{1} p_{2}}(\xi)$ by Eq. (A.1) and integrating over the horizon results in 


$$
\begin{aligned}
& \int_{H_{\mathbf{x}}} g^{p_{1} p_{2}}(\xi)(f(\mathbf{x}+\xi)-f(\mathbf{x})) \mathrm{d} V^{\prime} \\
& =\int_{H_{\mathbf{x}}} \xi_{1} \frac{\partial f(\mathbf{x})}{\partial x_{1}} g^{p_{1} p_{2}}(\xi) \mathrm{d} V^{\prime}+\int_{H_{\mathbf{x}}} \xi_{2} \frac{\partial f(\mathbf{x})}{\partial x_{2}} g^{p_{1} p_{2}}(\xi) \mathrm{d} V^{\prime} \\
& +\int_{H_{\mathbf{x}}} \frac{1}{2} \xi_{1}^{2} \frac{\partial^{2} f(\mathbf{x})}{\partial x_{1}^{2}} g^{p_{1} p_{2}}(\xi) \mathrm{d} V^{\prime}+\int_{H_{\mathbf{x}}} \frac{1}{2} \xi_{2}^{2} \frac{\partial^{2} f(\mathbf{x})}{\partial x_{2}^{2}} g^{p_{1} p_{2}}(\xi) \mathrm{d} V^{\prime} \\
& +\int_{H_{\mathbf{x}}} \xi_{1} \xi_{2} \frac{\partial^{2} f(\mathbf{x})}{\partial x_{1} \partial x_{2}} g^{p_{1} p_{2}}(\xi) \mathrm{d} V^{\prime}
\end{aligned}
$$

where $0<p_{1}+p_{2} \leq 2$. By enforcing the orthogonality conditions in Eq. (6) for each PD differential operator with as for $g^{10}(\xi)$ :

$$
\begin{aligned}
& \int_{H_{\mathrm{x}}} \xi_{1} g^{10}(\xi) \mathrm{d} V^{\prime}=1, \int_{H_{\mathrm{x}}} \xi_{2} g^{10}(\xi) \mathrm{d} V^{\prime}=0, \int_{H_{\mathrm{x}}} \frac{1}{2} \xi_{1}^{2} g^{10}(\xi) \mathrm{d} V^{\prime}=0, \\
& \int_{H_{\mathrm{x}}} \frac{1}{2} \xi_{2}^{2} g^{10}(\xi) \mathrm{d} V^{\prime}=0, \int_{H_{\mathrm{x}}} \xi_{1} \xi_{2} g^{10}(\xi) \mathrm{d} V^{\prime}=0
\end{aligned}
$$

for $g^{01}(\xi)$ :

$$
\begin{aligned}
& \int_{H_{\mathbf{x}}} \xi_{1} g^{01}(\xi) \mathrm{d} V^{\prime}=0, \int_{H_{\mathbf{x}}} \xi_{2} g^{01}(\xi) \mathrm{d} V^{\prime}=1, \int_{H_{\mathbf{x}}} \frac{1}{2} \xi_{1}^{2} g^{01}(\xi) \mathrm{d} V^{\prime}=0, \\
& \int_{H_{\mathbf{x}}} \frac{1}{2} \xi_{2}^{2} g^{01}(\xi) \mathrm{d} V^{\prime}=0, \int_{H_{\mathbf{x}}} \xi_{1} \xi_{2} g^{01}(\xi) \mathrm{d} V^{\prime}=0
\end{aligned}
$$

for $g^{20}(\xi)$ :

$$
\begin{aligned}
& \int_{H_{\mathrm{x}}} \xi_{1} g^{20}(\xi) \mathrm{d} V^{\prime}=0, \int_{H_{\mathrm{x}}} \xi_{2} g^{20}(\xi) \mathrm{d} V^{\prime}=0, \int_{H_{\mathrm{x}}} \frac{1}{2} \xi_{1}^{2} g^{20}(\xi) \mathrm{d} V^{\prime}=1 \\
& \int_{H_{\mathrm{x}}} \frac{1}{2} \xi_{2}^{2} g^{20}(\xi) \mathrm{d} V^{\prime}=0, \int_{H_{\mathrm{x}}} \xi_{1} \xi_{2} g^{20}(\xi) \mathrm{d} V^{\prime}=0
\end{aligned}
$$

for $g^{02}(\xi)$ :

$$
\begin{aligned}
& \int_{H_{\mathrm{x}}} \xi_{1} g^{02}(\xi) \mathrm{d} V^{\prime}=0, \int_{H_{\mathrm{x}}} \xi_{2} g^{02}(\xi) \mathrm{d} V^{\prime}=0, \int_{H_{\mathrm{x}}} \frac{1}{2} \xi_{1}^{2} g^{02}(\xi) \mathrm{d} V^{\prime}=0 \\
& \int_{H_{\mathrm{x}}} \frac{1}{2} \xi_{2}^{2} g^{02}(\xi) \mathrm{d} V^{\prime}=1, \int_{H_{\mathrm{x}}} \xi_{1} \xi_{2} g^{02}(\xi) \mathrm{d} V^{\prime}=0
\end{aligned}
$$

for $g^{11}(\xi)$ :

$$
\begin{aligned}
& \int_{H_{\mathrm{x}}} \xi_{1} g^{11}(\xi) \mathrm{d} V^{\prime}=0, \int_{H_{\mathrm{x}}} \xi_{2} g^{11}(\xi) \mathrm{d} V^{\prime}=0, \int_{H_{\mathrm{x}}} \frac{1}{2} \xi_{1}^{2} g^{11}(\xi) \mathrm{d} V^{\prime}=0, \\
& \int_{H_{\mathrm{x}}} \frac{1}{2} \xi_{2}^{2} g^{11}(\xi) \mathrm{d} V^{\prime}=0, \int_{H_{\mathrm{x}}} \xi_{1} \xi_{2} g^{11}(\xi) \mathrm{d} V^{\prime}=1
\end{aligned}
$$

the following relations can be obtained from Eq. (A.4) as 


$$
\begin{array}{ll}
\text { for } g^{10}(\xi): & \\
\text { for } g^{01}(\xi): & \int_{H_{\mathbf{x}}} g^{10}(\xi)(f(\mathbf{x}+\xi)-f(\mathbf{x})) \mathrm{d} V^{\prime}=\frac{\partial f(\mathbf{x})}{\partial x_{1}} \\
\text { for } g^{20}(\xi): & \int_{H_{\mathbf{x}}} g^{01}(\xi)(f(\mathbf{x}+\xi)-f(\mathbf{x})) \mathrm{d} V^{\prime}=\frac{\partial f(\mathbf{x})}{\partial x_{2}} \\
\text { for } g^{02}(\xi): & \int_{H_{\mathbf{x}}} g^{20}(\xi)(f(\mathbf{x}+\xi)-f(\mathbf{x})) \mathrm{d} V^{\prime}=\frac{\partial^{2} f(\mathbf{x})}{\partial x_{1}^{2}} \\
\text { for } g^{11}(\xi): & \int_{H_{\mathbf{x}}} g^{02}(\xi)(f(\mathbf{x}+\xi)-f(\mathbf{x})) \mathrm{d} V^{\prime}=\frac{\partial^{2} f(\mathbf{x})}{\partial x_{2}^{2}} \\
& \int_{H_{\mathbf{x}}} g^{11}(\xi)(f(\mathbf{x}+\xi)-f(\mathbf{x})) \mathrm{d} V^{\prime}=\frac{\partial^{2} f(\mathbf{x})}{\partial x_{1} \partial x_{2}}
\end{array}
$$

In conclusion, the differentials in local form are reformulated into their non-local form as

$$
\left\{\begin{array}{l}
\frac{\partial f(\mathbf{x})}{\partial x_{1}} \\
\frac{\partial f(\mathbf{x})}{\partial x_{2}} \\
\frac{\partial^{2} f(\mathbf{x})}{\partial x_{1}^{2}} \\
\frac{\partial^{2} f(\mathbf{x})}{\partial x_{2}^{2}} \\
\frac{\partial^{2} f(\mathbf{x})}{\partial x_{1} \partial x_{2}}
\end{array}\right\}=\int_{H_{\mathbf{x}}}(f(\mathbf{x}+\xi)-f(\mathbf{x}))\left\{\begin{array}{l}
g^{10}(\xi) \\
g^{01}(\xi) \\
g^{20}(\xi) \\
g^{02}(\xi) \\
g^{11}(\xi)
\end{array}\right\} \mathrm{d} V^{\prime}
$$

where the term $g$ represents the PD differential operator for each derivative, such as $g^{10}(\xi)$ is the PD differential operator for $\partial f(\mathbf{x}) / \partial x_{1}$.

\section{Expressions for PD Differential Operators}

As provided in Eq. (11), differential operator functions, $g^{p_{1} p_{2}}(\xi)$ are chosen as

for $g^{10}(\xi): \quad g^{10}(\xi)=\left(a_{10}^{10}\right) w \xi_{1}+\left(a_{01}^{10}\right) w \xi_{2}+\left(a_{20}^{10}\right) w \xi_{1}^{2}+\left(a_{02}^{10}\right) w \xi_{2}^{2}+\left(a_{11}^{10}\right) w \xi_{1} \xi_{2}$

for $g^{01}(\xi): \quad g^{01}(\xi)=\left(a_{10}^{01}\right) w \xi_{1}+\left(a_{01}^{01}\right) w \xi_{2}+\left(a_{20}^{01}\right) w \xi_{1}^{2}+\left(a_{02}^{01}\right) w \xi_{2}^{2}+\left(a_{11}^{01}\right) w \xi_{1} \xi_{2}$

for $g^{20}(\xi): \quad g^{20}(\xi)=\left(a_{10}^{20}\right) w \xi_{1}+\left(a_{01}^{20}\right) w \xi_{2}+\left(a_{20}^{20}\right) w \xi_{1}^{2}+\left(a_{02}^{20}\right) w \xi_{2}^{2}+\left(a_{11}^{20}\right) w \xi_{1} \xi_{2}$

for $g^{02}(\xi): \quad g^{02}(\xi)=\left(a_{10}^{02}\right) w \xi_{1}+\left(a_{01}^{02}\right) w \xi_{2}+\left(a_{20}^{02}\right) w \xi_{1}^{2}+\left(a_{02}^{02}\right) w \xi_{2}^{2}+\left(a_{11}^{02}\right) w \xi_{1} \xi_{2}$

for $g^{11}(\xi): \quad g^{11}(\xi)=\left(a_{10}^{11}\right) w \xi_{1}+\left(a_{01}^{11}\right) w \xi_{2}+\left(a_{20}^{11}\right) w \xi_{1}^{2}+\left(a_{02}^{11}\right) w \xi_{2}^{2}+\left(a_{11}^{11}\right) w \xi_{1} \xi_{2}$ 
or expressed in a compact form as

$$
\left\{\begin{array}{l}
g^{10}(\xi) \\
g^{01}(\xi) \\
g^{20}(\xi) \\
g^{02}(\xi) \\
g^{11}(\xi)
\end{array}\right\}=\left[\begin{array}{lllll}
a_{10}^{10} & a_{01}^{10} & a_{20}^{10} & a_{02}^{10} & a_{11}^{10} \\
a_{10}^{01} & a_{01}^{01} & a_{20}^{01} & a_{02}^{01} & a_{11}^{01} \\
a_{10}^{20} & a_{01}^{20} & a_{20}^{20} & a_{02}^{20} & a_{11}^{20} \\
a_{10}^{02} & a_{01}^{02} & a_{20}^{02} & a_{02}^{02} & a_{11}^{02} \\
a_{10}^{11} & a_{01}^{11} & a_{20}^{11} & a_{02}^{11} & a_{11}^{11}
\end{array}\right]\left\{\begin{array}{c}
w \xi_{1} \\
w \xi_{2} \\
w \xi_{1}^{2} \\
w \xi_{2}^{2} \\
w \xi_{1} \xi_{2}
\end{array}\right\}
$$

where $a_{q_{1} q_{2}}^{p_{1} p_{2}}$ represents the coefficients of the polynomials with $0<q_{1}+q_{2} \leq 2$.

By substituting Eq. (A.8a) into orthogonality conditions provided in Eq. (A.5a) the following equations for $g^{10}(\xi)$ can be obtained as

$$
\begin{aligned}
& \left(a_{10}^{10}\right) \int_{H_{\mathbf{x}}} w \xi_{1}^{2} \mathrm{~d} V^{\prime}+\left(a_{01}^{10}\right) \int_{H_{\mathbf{x}}} w \xi_{1} \xi_{2} \mathrm{~d} V^{\prime}+\left(a_{20}^{10}\right) \int_{H_{\mathbf{x}}} w \xi_{1}^{3} \mathrm{~d} V^{\prime} \\
& +\left(a_{02}^{10}\right) \int_{H_{\mathbf{x}}} w \xi_{1} \xi_{2}^{2} \mathrm{~d} V^{\prime}+\left(a_{11}^{10}\right) \int_{H_{\mathbf{x}}} w \xi_{1}^{2} \xi_{2} \mathrm{~d} V^{\prime}=1 \\
& \left(a_{10}^{10}\right) \int_{H_{\mathbf{x}}} w \xi_{1} \xi_{2} \mathrm{~d} V^{\prime}+\left(a_{01}^{10}\right) \int_{H_{\mathbf{x}}} w \xi_{2}^{2} \mathrm{~d} V^{\prime}+\left(a_{20}^{10}\right) \int_{H_{\mathbf{x}}} w \xi_{1}^{2} \xi_{2} \mathrm{~d} V^{\prime} \\
& +\left(a_{02}^{10}\right) \int_{H_{\mathbf{x}}} w \xi_{2}^{3} \mathrm{~d} V^{\prime}+\left(a_{11}^{10}\right) \int_{H_{\mathbf{x}}} w \xi_{1} \xi_{2}^{2} \mathrm{~d} V^{\prime}=0 \\
& \left(a_{10}^{10}\right) \int_{H_{\mathbf{x}}} w \xi_{1}^{3} \mathrm{~d} V^{\prime}+\left(a_{01}^{10}\right) \int_{H_{\mathbf{x}}} w \xi_{1}^{2} \xi_{2} \mathrm{~d} V^{\prime}+\left(a_{20}^{10}\right) \int_{H_{\mathbf{x}}} w \xi_{1}^{4} \mathrm{~d} V^{\prime} \\
& +\left(a_{02}^{10}\right) \int_{H_{\mathbf{x}}} w \xi_{1}^{2} \xi_{2}^{2} \mathrm{~d} V^{\prime}+\left(a_{11}^{10}\right) \int_{H_{\mathbf{x}}} w \xi_{1}^{3} \xi_{2} \mathrm{~d} V^{\prime}=0 \\
& \left(a_{10}^{10}\right) \int_{H_{\mathbf{x}}} w \xi_{1} \xi_{2}^{2} \mathrm{~d} V^{\prime}+\left(a_{01}^{10}\right) \int_{H_{\mathbf{x}}} w \xi_{2}^{3} \mathrm{~d} V^{\prime}+\left(a_{20}^{10}\right) \int_{H_{\mathbf{x}}} w \xi_{1}^{2} \xi_{2}^{2} \mathrm{~d} V^{\prime} \\
& +\left(a_{02}^{10}\right) \int_{H_{\mathbf{x}}} w \xi_{2}^{4} \mathrm{~d} V^{\prime}+\left(a_{11}^{10}\right) \int_{H_{\mathbf{x}}} w \xi_{1} \xi_{2}^{3} \mathrm{~d} V^{\prime}=0 \\
& \left(a_{10}^{10}\right) \int_{H_{\mathbf{x}}} w \xi_{1}^{2} \xi_{2} \mathrm{~d} V^{\prime}+\left(a_{01}^{10}\right) \int_{H_{\mathbf{x}}} w \xi_{1} \xi_{2}^{2} \mathrm{~d} V^{\prime}+\left(a_{20}^{10}\right) \int_{H_{\mathbf{x}}} w \xi_{1}^{3} \xi_{2} \mathrm{~d} V^{\prime} \\
& +\left(a_{02}^{10}\right) \int_{H_{\mathbf{x}}} w \xi_{1} \xi_{2}^{3} \mathrm{~d} V^{\prime}+\left(a_{11}^{10}\right) \int_{H_{\mathbf{x}}} w \xi_{1}^{2} \xi_{2}^{2} \mathrm{~d} V^{\prime}=0
\end{aligned}
$$

Similarly, by substituting Eq. (A.8b-e) into orthogonality conditions provided in Eq. (A.5b-e) following relations can be obtained

$$
\mathbf{A a}=\mathbf{b}
$$

with

$$
\mathbf{A}=\int_{H_{x}}\left[\begin{array}{ccccc}
w \xi_{1}^{2} & w \xi_{1} \xi_{2} & w \xi_{1}^{3} & w \xi_{1} \xi_{2}^{2} & w \xi_{1}^{2} \xi_{2} \\
w \xi_{1} \xi_{2} & w \xi_{2}^{2} & w \xi_{1}^{2} \xi_{2} & w \xi_{2}^{3} & w \xi_{1} \xi_{2}^{2} \\
w \xi_{1}^{3} & w \xi_{1}^{2} \xi_{2} & w \xi_{1}^{4} & w \xi_{1}^{2} \xi_{2}^{2} & w \xi_{1}^{3} \xi_{2} \\
w \xi_{1} \xi_{2}^{2} & w \xi_{2}^{3} & w \xi_{1}^{2} \xi_{2}^{2} & w \xi_{2}^{4} & w \xi_{1} \xi_{2}^{3} \\
w \xi_{1}^{2} \xi_{2} & w \xi_{1} \xi_{2}^{2} & w \xi_{1}^{3} \xi_{2} & w \xi_{1} \xi_{2}^{3} & w \xi_{1}^{2} \xi_{2}^{2}
\end{array}\right] \mathrm{d} V^{\prime}
$$




$$
\begin{gathered}
\mathbf{a}=\left[\begin{array}{lllll}
a_{10}^{10} & a_{10}^{01} & a_{10}^{20} & a_{10}^{02} & a_{10}^{11} \\
a_{01}^{10} & a_{01}^{01} & a_{01}^{20} & a_{01}^{02} & a_{01}^{11} \\
a_{20}^{10} & a_{20}^{01} & a_{20}^{20} & a_{20}^{02} & a_{20}^{11} \\
a_{02}^{10} & a_{02}^{01} & a_{02}^{20} & a_{02}^{02} & a_{02}^{11} \\
a_{11}^{10} & a_{11}^{01} & a_{11}^{20} & a_{11}^{02} & a_{11}^{11}
\end{array}\right] \\
\mathbf{b}=\left[\begin{array}{lllll}
1 & 0 & 0 & 0 & 0 \\
0 & 1 & 0 & 0 & 0 \\
0 & 0 & 2 & 0 & 0 \\
0 & 0 & 0 & 2 & 0 \\
0 & 0 & 0 & 0 & 1
\end{array}\right]
\end{gathered}
$$

After solving for the unknown coefficient $\mathbf{a}$, the non-local form of derivatives of function $f$ can be found.

\section{Analytical Solution}

For 2D PD differential operator, the integration domain, $H_{\mathrm{x}}$, is set to be a disk with its radius being horizon $\delta$ and thickness being $h$, analytical form of PD differential operator can also be calculated.

By utilizing the weighted function

$$
w(\xi)=e^{-(2 \xi / \delta)^{2}}
$$

and by substituting $\xi=|\xi|, \xi_{1}=\xi \cos (\theta)$, and $\xi_{2}=\xi \sin (\theta)$ with $\theta$ being the bond direction with respect to the positive $x_{1}$ direction into Eq.(A.12a), the analytical form of shape matrix, A, can be obtained as

$$
\mathbf{A}=\int_{0}^{2 \pi} \int_{0}^{\delta} e^{-(2 \xi / \delta)^{2}}\left[\begin{array}{ccccc}
\xi^{2} \mathrm{c}^{2} & \xi^{2} \mathrm{cs} & \xi^{3} \mathrm{c}^{3} & \xi^{3} \mathrm{cs}^{2} & \xi^{3} \mathrm{c}^{2} \mathrm{~s} \\
\xi^{2} \mathrm{cs} & \xi^{2} \mathrm{~s}^{2} & \xi^{3} \mathrm{c}^{2} \mathrm{~s} & \xi^{3} \mathrm{~s}^{3} & \xi^{3} \mathrm{cs} \\
\xi^{3} \mathrm{c}^{3} & \xi^{3} \mathrm{c}^{2} \mathrm{~s} & \xi^{4} \mathrm{c}^{4} & \xi^{4} \mathrm{c}^{2} \mathrm{~s}^{2} & \xi^{4} \mathrm{c}^{3} \mathrm{~s} \\
\xi^{3} \mathrm{cs} \mathrm{s}^{2} & \xi^{3} \mathrm{~s}^{3} & \xi^{4} \mathrm{c}^{2} \mathrm{~s}^{2} & \xi^{4} \mathrm{~s}^{4} & \xi^{4} \mathrm{cs} \\
\xi^{3} \mathrm{c}^{2} \mathrm{~s} & \xi^{3} \mathrm{cs}^{2} & \xi^{4} \mathrm{c}^{3} \mathrm{~s} & \xi^{4} \mathrm{cs}^{3} & \xi^{4} \mathrm{c}^{2} \mathrm{~s}^{2}
\end{array}\right] h \mathrm{~d} \mathrm{~d} \theta
$$

where $\mathrm{c}=\cos (\theta)$ and $\mathrm{s}=\sin (\theta)$. After performing the integrations, Eq.(A.14) results in 


$$
\mathbf{A}=\frac{\pi h \delta^{4}}{32 e^{4}}\left[\begin{array}{ccccc}
\left(e^{4}-5\right) & 0 & 0 & 0 & 0 \\
0 & \left(e^{4}-5\right) & 0 & 0 & 0 \\
0 & 0 & \frac{3\left(e^{4}-13\right) \delta^{2}}{8} & \frac{\left(e^{4}-13\right) \delta^{2}}{8} & 0 \\
0 & 0 & \frac{\left(e^{4}-13\right) \delta^{2}}{8} & \frac{3\left(e^{4}-13\right) \delta^{2}}{8} & 0 \\
0 & 0 & 0 & 0 & \frac{\left(e^{4}-13\right) \delta^{2}}{8}
\end{array}\right]
$$

After substituting $\mathbf{A}$ into Eq. (A.11), the expression of matrix $\mathbf{a}$ can be obtained as

$$
\mathbf{a}=\frac{32 e^{4}}{\pi h \delta^{4}}\left[\begin{array}{ccccc}
\frac{1}{\left(e^{4}-5\right)} & 0 & 0 & 0 & 0 \\
0 & \frac{1}{\left(e^{4}-5\right)} & 0 & 0 & 0 \\
0 & 0 & \frac{6}{\left(e^{4}-13\right) \delta^{2}} & \frac{-2}{\left(e^{4}-13\right) \delta^{2}} & 0 \\
0 & 0 & \frac{-2}{\left(e^{4}-13\right) \delta^{2}} & \frac{6}{\left(e^{4}-13\right) \delta^{2}} & 0 \\
0 & 0 & 0 & 0 & \frac{8}{\left(e^{4}-13\right) \delta^{2}}
\end{array}\right]
$$

Consequently, by substituting Eq. (A.13) and Eq. (A.16) into Eq.(A.9), the analytical expression of the PD differential operator for the $2 \mathrm{D}$ problem is obtained as

$$
\left\{\begin{array}{l}
g^{10}(\xi) \\
g^{01}(\xi) \\
g^{20}(\xi) \\
g^{02}(\xi) \\
g^{11}(\xi)
\end{array}\right\}=\frac{32 \xi e^{-(2 \xi / \delta)^{2}+4}}{\pi h \delta^{4}}\left\{\begin{array}{c}
\frac{\cos (\theta)}{\left(e^{4}-5\right)} \\
\frac{\sin (\theta)}{\left(e^{4}-5\right)} \\
\frac{2 \xi\left(4 \cos ^{2}(\theta)-1\right)}{\left(e^{4}-13\right) \delta^{2}} \\
\frac{2 \xi\left(4 \sin ^{2}(\theta)-1\right)}{\left(e^{4}-13\right) \delta^{2}} \\
\frac{8 \xi \cos (\theta) \sin (\theta)}{\left(e^{4}-13\right) \delta^{2}}
\end{array}\right\}
$$

Finally, the partial derivatives will be converted into their non-local forms as 


$$
\left\{\begin{array}{c}
\frac{\partial f(\mathbf{x})}{\partial x_{1}} \\
\frac{\partial f(\mathbf{x})}{\partial x_{2}} \\
\frac{\partial^{2} f(\mathbf{x})}{\partial x_{1}^{2}} \\
\frac{\partial^{2} f(\mathbf{x})}{\partial x_{2}^{2}} \\
\frac{\partial^{2} f(\mathbf{x})}{\partial x_{1} \partial x_{2}}
\end{array}\right\}=\int_{H_{\mathbf{x}}} \frac{32 \xi e^{-(2 \xi / \delta)^{2}+4}}{\pi h \delta^{4}}\left\{\begin{array}{c}
\frac{\cos (\theta)}{\left(e^{4}-5\right)} \\
\frac{\sin (\theta)}{\left(e^{4}-5\right)} \\
\frac{2 \xi\left(4 \cos ^{2}(\theta)-1\right)}{\left(e^{4}-13\right) \delta^{2}} \\
\frac{8 \xi \cos (\theta)-1)}{\left(e^{4}-13\right) \delta^{2}}
\end{array}\right\}(f(\mathbf{x}+\xi)-f(\mathbf{x})) \mathrm{d} V^{\prime}
$$

It should be mentioned that for the numerical simulations, PD differential operator is computed numerically.

\section{References}

Adami, S., Hu, X.Y., Adams, N.A., 2012. A generalized wall boundary condition for smoothed particle hydrodynamics. Journal Of Computational Physics 231 (21), 7057-7075.

Adami, S., Hu, X.Y., Adams, N.A., 2013. A transport-velocity formulation for smoothed particle hydrodynamics. Journal Of Computational Physics 241, 292-307.

Anderson, E., Bai, Z., Dongarra, J., Greenbaum, A., McKenney, A., Du Croz, J., Hammarling, S., Demmel, J., Bischof, C., Sorensen, D., 1990. LAPACK: A portable linear algebra library for highperformance computers, Proceedings of the 1990 ACM/IEEE conference on Supercomputing. IEEE Computer Society Press, pp. 2-11.

Anderson, J.D., Wendt, J., 1995. Computational fluid dynamics. Springer.

Batchelor, G.K., 2000. An introduction to fluid dynamics. Cambridge university press.

Becker, M., Teschner, M., 2007. Weakly compressible SPH for free surface flows, Proceedings of the 2007 ACM SIGGRAPH/Eurographics symposium on Computer animation. Eurographics Association, pp. 209-217.

Bergel, G.L., Li, S., 2016. The total and updated lagrangian formulations of state-based peridynamics. Computational Mechanics 58 (2), 351-370.

Cao, X.Y., Ming, F.R., Zhang, A.M., 2014. Sloshing in a rectangular tank based on SPH simulation. Applied Ocean Research 47, 241-254.

Chaniotis, A.K., Poulikakos, D., Koumoutsakos, P., 2002. Remeshed smoothed particle hydrodynamics for the simulation of viscous and heat conducting flows. Journal Of Computational Physics 182 (1), 67 90 .

Colagrossi, A., Landrini, M., 2003. Numerical simulation of interfacial flows by smoothed particle hydrodynamics. Journal Of Computational Physics 191 (2), 448-475.

Drikakis, D., Rider, W., 2005. The artificial compressibility method. High-Resolution Methods for Incompressible and Low-Speed Flows, 173-208.

Faltinsen, O., 1993. Sea loads on ships and offshore structures. Cambridge university press.

Gingold, R.A., Monaghan, J.J., 1977. Smoothed particle hydrodynamics: theory and application to nonspherical stars. Monthly notices of the royal astronomical society 181 (3), 375-389.

Gómez-Gesteira, M., Cerqueiro, D., Crespo, C., Dalrymple, R.A., 2005. Green water overtopping analyzed with a SPH model. Ocean Engineering 32 (2), 223-238.

Gu, X., Madenci, E., Zhang, Q., 2018. Revisit of non-ordinary state-based peridynamics. Engineering Fracture Mechanics 190, 31-52. 
Intel, R., 2011. Intel math kernel library reference manual. Tech. Rep. 630813-051US, 2012.[Online]. Available: http://software. intel. com/sites/products/documentation/hpc/mkl/mklman/mklman. pdf.

Katiyar, A., Foster, J.T., Ouchi, H., Sharma, M.M., 2014. A peridynamic formulation of pressure driven convective fluid transport in porous media. Journal Of Computational Physics 261, 209-229.

Koshizuka, S., 1995. A particle method for incompressible viscous flow with fluid fragmentation. Comput. Fluid Dyn. J. 4, 29.

Koshizuka, S., Oka, Y., 1996. Moving-Particle Semi-Implicit Method for Fragmentation of Incompressible Fluid. Nuclear Science and Engineering 123 (3), 421-434.

Liu, G.R.L.M.B., 2011. SPH for General Dynamic Fluid Flows, Smoothed Particle Hydrodynamics. WORLD SCIENTIFIC, pp. 103-176.

Liu, R.W., Xue, Y.Z., Lu, X.K., Cheng, W.X., 2018. Simulation of ship navigation in ice rubble based on peridynamics. Ocean Engineering 148, 286-298.

Lucy, L.B., 1977. A numerical approach to the testing of the fission hypothesis. The astronomical journal 82, 1013-1024.

Luo, J., Sundararaghavan, V., 2018. Stress-point method for stabilizing zero-energy modes in nonordinary state-based peridynamics. International Journal Of Solids and Structures 150, 197-207.

Macek, R.W., Silling, S.A., 2007. Peridynamics via finite element analysis. Finite Elements In Analysis and Design 43 (15), 1169-1178.

Madenci, E., 2017. Peridynamic integrals for strain invariants of homogeneous deformation. ZAMM -

Journal of Applied Mathematics and Mechanics/Zeitschrift für Angewandte Mathematik und Mechanik, 97(10), pp.1236-1251.

Madenci, E., Barut, A., Futch, M., 2016. Peridynamic differential operator and its applications. Computer Methods In Applied Mechanics and Engineering 304, 408-451.

Madenci, E., Oterkus, E., 2014. Peridynamic theory and its applications. Springer.

Mao, Z., Liu, G.R., 2018. A Lagrangian gradient smoothing method for solid-flow problems using simplicial mesh. International Journal For Numerical Methods In Engineering 113 (5), 858-890.

Martin, J.C., Moyce, W.J., Penney, W.G., Price, A., Thornhill, C., 1952. Part IV. An experimental study of the collapse of liquid columns on a rigid horizontal plane. Phil. Trans. R. Soc. Lond. A 244 (882), 312-324.

Monaghan, J.J., 1992. Smoothed particle hydrodynamics. Annual review of astronomy and astrophysics 30 (1), 543-574.

Monaghan, J.J., 1994. Simulating Free Surface Flows with SPH. Journal Of Computational Physics 110 (2), 399-406.

Morris, J.P., Fox, P.J., Zhu, Y., 1997. Modeling Low Reynolds Number Incompressible Flows Using SPH. Journal Of Computational Physics 136 (1), 214-226.

Oterkus, E., Madenci, E., 2012a. Peridynamic Analysis of Fiber-Reinforced Composite Materials. Journal of Mechanics of Materials and Structures 7 (1), 45-84.

Oterkus, E., Madenci, E., 2012b. Peridynamic Theory for Damage Initiation and Growth in Composite Laminate. Key Engineering Materials 488-489, 355-358.

Oterkus, S., 2015. Peridynamics for the solution of multiphysics problems. The University of Arizona, Arizona.

Oterkus, S., Madenci, E., 2015. Peridynamics for Antiplane Shear and Torsional Deformations. Journal of Mechanics of Materials and Structures 10 (2), 167-193.

Oterkus, S., Madenci, E., 2017. Peridynamic modeling of fuel pellet cracking. Engineering Fracture Mechanics 176, 23-37.

Oterkus, S., Madenci, E., Agwai, A., 2014. Peridynamic thermal diffusion. Journal Of Computational Physics 265, 71-96.

Oterkus, S., Madenci, E., Oterkus, E., 2017. Fully coupled poroelastic peridynamic formulation for fluid-filled fractures. Engineering Geology 225, 19-28.

Ouchi, H., Katiyar, A., York, J., Foster, J., Sharma, M.M., 2015. A fully coupled porous flow and geomechanics model for fluid driven cracks: a peridynamics approach. Computational Mechanics 55 (3), 561-576.

Silling, S.A., 2000. Reformulation of elasticity theory for discontinuities and long-range forces. Journal Of The Mechanics and Physics Of Solids 48 (1), 175-209. 
Silling, S.A., 2017. Stability of peridynamic correspondence material models and their particle discretizations. Computer Methods In Applied Mechanics and Engineering 322, 42-57.

Silling, S.A., Askari, E., 2005. A meshfree method based on the peridynamic model of solid mechanics. Computers \& Structures 83 (17-18), 1526-1535.

Silling, S.A., Epton, M., Weckner, O., Xu, J., Askari, E., 2007. Peridynamic states and constitutive modeling. Journal Of Elasticity 88 (2), 151-184.

Silling, S.A., Parks, M.L., Kamm, J.R., Weckner, O., Rassaian, M., 2017. Modeling shockwaves and impact phenomena with Eulerian peridynamics. International Journal Of Impact Engineering 107, 4757.

Swope, W.C., Andersen, H.C., Berens, P.H., Wilson, K.R., 1982. A computer simulation method for the calculation of equilibrium constants for the formation of physical clusters of molecules: Application to small water clusters. The Journal of Chemical Physics 76 (1), 637-649.

Tu, Q.S., Li, S.F., 2017. An updated Lagrangian particle hydrodynamics (ULPH) for Newtonian fluids. Journal Of Computational Physics 348, 493-513.

Wang, C., Xiong, W.P., Chang, X., Ye, L.Y., Li, X., 2018. Analysis of variable working conditions for propeller-ice interaction. Ocean Engineering 156, 277-293.

Wang, J., Zhang, X., 2018. Modified Particle Method with integral Navier-Stokes formulation for incompressible flows. Journal Of Computational Physics 366, 1-13.

Ye, L.Y., Wang, C., Chang, X., Zhang, H.Y., 2017. Propeller-ice contact modeling with peridynamics. Ocean Engineering 139, 54-64. 